\title{
Millimetre Wave Frequency Band as a Candidate Spectrum for 5G Network Architecture: A Survey
}

\author{
Naser Al-Falahy ${ }^{(1),(2)}$ and Omar Y. K. Alani ${ }^{(1)}$ \\ (1) University of Salford, Manchester, United Kingdom \\ The Crescent, Salford M54WT \\ (2) University of Anbar, Iraq. \\ N.F.A.AL-Falahy@edu.salford.ac.uk, O.Y.K.Alani@salford.ac.uk
}

\begin{abstract}
In order to meet the huge growth in global mobile data traffic in 2020 and beyond, the development of the $5^{\text {th }}$ Generation $(5 \mathrm{G})$ system is required as the current $4 \mathrm{G}$ system is expected to fall short of the provision needed for such growth. $5 \mathrm{G}$ is anticipated to use a higher carrier frequency in the millimetre wave (mm-wave) band, within the 20 to $90 \mathrm{GHz}$, due to the availability of a vast amount of unexploited bandwidth. It is a revolutionary step to use these bands because of their different propagation characteristics, severe atmospheric attenuation, and hardware constraints. In this paper, we carry out a survey of $5 \mathrm{G}$ research contributions and proposed design architectures based on mm-wave communications. We present and discuss the use of mm-wave as indoor and outdoor mobile access, as a wireless backhaul solution, and as a key enabler for higher order sectorisation. Wireless standards such as IEE802.11ad, which are operating in mm-wave band have been presented. These standards have been designed for short range, ultra high data throughput systemS in the $60 \mathrm{GHz}$ band. Furthermore, this survey provides new insights regarding relevant and open issues in

adopting mm-wave for $5 \mathrm{G}$ networks. This includes increased handoff rate and interference in Ultra-Dense Network (UDN), waveform consideration with higher spectral efficiency, and supporting spatial multiplexing in mm-wave line of site. This survey also introduces a distributed base station architecture in mm-wave as an approach to address increased handoff rate in UDN, and to provide an alternative way for network densification in a time and cost effective manner.
\end{abstract}

Keywords - 5G network; millimetre wave survey; network densification; sectorisation; distributed base station.

\section{Introduction}

The vast proliferation and the enormous use of data-hungry devices such as smart-phones and laptops have dramatically increased Global Mobile Data Traffic (GMDT). GMDT already grew up to 74\% in 2015, reaching 3.7 exabytes per month. This figure is forecasted to grow by 2020 to 30.6 exabytes [1]. The fourth generation (4G) system was not designed to cope with this huge growth. Therefore, the next generation of wireless standards needs to extend its bandwidth into a higher carrier frequency in the millimetre wave (mm-wave) band ranging from 3 to $300 \mathrm{GHz}$.

The spectrum at microwave bands (MW) is becoming too scarce, because almost all cellular communication systems are operating in the sub $3 \mathrm{GHz}$ band. Therefore, moving to the mm-wave bands is essential because there are wide unused bandwidths, particularly from 20 to $90 \mathrm{GHz}$. These bands could become accessible for the Fifth Generation (5G) system as a potential solution for achieving a 1000 folds capacity increase compared to the current Long Term Evolution Advance (LTE-A) networks [2][3]. The mm-wave spectrum refers to the frequencies from 30 to $300 \mathrm{GHz}$, which is called the extremely high frequency (EHF) band. The $3-30 \mathrm{GHz}$ spectrum is called the Super High Frequency (SHF) centimetre wave band. Because EHF and SHF bands have approximately similar propagation conditions, the 3-300GHz spectrum is collectively called the mm-wave band with their wavelengths ranging from 1 to $100 \mathrm{~mm}$ [4]. The high speed data rate and low end-to-end latency requirements cannot be fulfilled with mere evolution from the existing $4 \mathrm{G}$ network or minor changes [5]. Therefore, researchers focus their attention on technologies that comprise major and radical changes in the base stations (nodes) level as well as at the network (core, backhaul) level, because only these types of changes have the capacity to meet these stringent requirements. In this context, the mm-wave band is the most prominent technology and the key enabler to satisfy the extreme demands of future applications.

In this paper, we have carried out a detailed survey regarding the use of the mm-wave band for cellular purposes by considering most of the recent research contributions and publications in this field. The focus is on the impact of using 
the mm-wave band in a new network architecture, which will require radical change at both network and component levels. New network design options have been proposed in many contributions in order to cope with the propagation characteristics of mm-wave. These include: higher order sectorisation, ultra-dense network implementation, and the use of distributed base station architectures. These architectures exploit the mm-wave to significantly boost the network capacity, and to cope with severe losses that characterise mm-wave propagation.

After presenting the potential bandwidth allocations in section 2, we have summarised the characteristics of mm-wave communications in section 3, which include high path loss and atmospheric attenuation. Due to their short wavelengths, mm-wave signals have severe propagation loss and scattering, and high sensitivity to blockage by obstacles such as buildings, street furniture, and human bodies. We then introduce the rain effect, as mm-wave signals can suffer significant attenuation in heavy rain as raindrops have approximately the same size as the signal wavelengths and thus result in severe signal attenuation due to scattering. One of the solutions to this loss is by reducing the Inter-Site-Distance (ISD), which will decrease the signal path by making the Access Point (AP) much closer to the users. After that, three wireless standards operating in the mm-wave band are introduced in section 4 . These standards are operating in the $60 \mathrm{GHz}$ band and suppose to provide very high data speed in short ranges. In section 5, we have identified the potential use of mm-wave in 5G, for indoor, outdoor, and backhaul solutions, and the contributions in each field are highlighted and discussed. Relevant issues to adopting mm-wave in $5 \mathrm{G}$ are discussed in section 6 , which include the handoff issue and interference in Ultra-Dense Network (UDN), waveform consideration, providing spatial multiplexing in Line of Site (LoS) transmission, supporting Machine-to-Machine (M2M) traffic, and introducing distributed base stations in the mmwave environment. Finally, the conclusions are drawn in section 7.

\section{Extending the operation to the mm-wave}

In this section, we summarise the frequency bands expected to be allocated for $5 \mathrm{G}$ communication systems, and present the potential move to a higher frequency band in the mm-wave bandwidth. For frequencies below $6 \mathrm{GHz}$, a maximum of $2.5 \mathrm{GHz}$ of licensed bandwidth might be potentially assigned with the largest part at 3.5GHz. Moving to the mm-wave band, in the United States, the Federal Communications Commission (FCC) [6] and the International Telecommunication Union-Radio (ITU-R) in World Radio-communication Conferences (WRC) proceedings are responsible for spectrum regulation and allocation [7]. For the 5G cellular network, many bands have been released located at 28-30 GHz, 38-40GHz, the free- licensed band $57-64 \mathrm{GHz}$, which has been extended to $71 \mathrm{GHz}$, with $14 \mathrm{GHz}$ of contiguous band, 15 times as much as all the Wi-Fi unlicensed spectrum [8]. There is also a 12.9GHz band from the E-band located at $71-76 \mathrm{GHz}, 81-86 \mathrm{GHz}$, and $92-95 \mathrm{GHz}$ [2][3]. In the UK, the pioneer band centred at $26 \mathrm{GHz}$ has been released by Ofcom [9] for potential 5G use ranging between $24.25-27.5 \mathrm{GHz}$. China has considered frequency bands at $45 \mathrm{GHz}$ for licensed and unlicensed communications systems and 5G considerations. Moreover, the 95$150 \mathrm{GHz}$ band could be used by licensed spectrum sharing (LSA). This will allow mobile networks to use bandwidth originally deployed for incumbent systems without harming their operation. Taking this into account, $1.177 \mathrm{MHz}$ of the International Mobile Telecommunications (IMT) band is in use, therefore a 3 to 10 fold increase is expected in allocated spectrum by 2020 or beyond [10]. These bands could become accessible for $5 \mathrm{G}$ as a potential solution for achieving the capacity increase compared to current LTE-A networks. The potential spectrum for 5G is shown in Fig.2.

Due to spectrum scarcity in the legacy spectrum below $3 \mathrm{GHz}$, the $5 \mathrm{G}$ system will extend its frequency to the mm-wave bands, particularly from 20 to $90 \mathrm{GHz}$, because there is a vast amount of unexploited bandwidth. This step is revolutionary because of the very different propagation rules, severe atmospheric attenuation, and hardware constraints that characterise mm-wave communication. These challenges, however, can be overcomed by using beamforming and larger scale antenna arrays. It is generally accepted that mm-wave communication is better when used with a small-cell radius below 200m, in order to reduce the high path loss and atmospheric attenuation (by making APs closer to the users). Fortunately, using dense mm-wave hotspots fits with the trend of current network densification by small-cells [4][11]. The adoption of mm-wave for cellular purposes will bring new challenges that need to be addressed, such as:

\subsection{High Path loss}

Channel measurements such as in [12] and [13] demonstrate that mm-wave is sensitive to blockages, with different path loss between LoS and None LoS(NLoS) transmissions. The mm-wave suffers significant penetration loss through solid materials. Additionally, heavy rain can cause considerable attenuation due to scattering, as rain-drops are comparable in size to the mm-wave wavelengths [4]. Therefore, LoS transmission is limited by the existence of blockages, and NLoS as a result of reflected waves from blockages surfaces are generally weak, but usually contribute to 
a good signal that covers users in shadow [13][14]. In general, LoS transmission is similar to the free space, with a path loss exponent of 2 [15], whereas for the NLoS link, the path loss exponent is shown in [12][15] to be larger compared with the LoS exponent, 3.86 measured at the University of Texas, Austin campus and 5.76 measured at NYC downtown. A recent path loss model for frequencies from 0.5 to $100 \mathrm{GHz}$ has been released by the 3rd Generation Partnership Project (3GPP) [16][17]. Therefore, the path loss on the link between 5G node and a device shown in Fig. 1 is defined by [16]:

$$
\mathrm{PL}_{1}=32.4+20 \log _{10}\left(f_{c}\right)+21 \log _{10}\left(d_{3 \mathrm{D}}\right)
$$

for LoS transmission, and:

$$
\mathrm{PL}_{2}=32.4+20 \log _{10}\left(f_{c}\right)+31.9 \log _{10}\left(d_{3 \mathrm{D}}\right)
$$

for NLoS, where: $f_{c}$ is the frequency in $\mathrm{GHz}, d_{3 D}$ is the separation between BS antenna and receiver antenna in metres. Fig. 3 shows the excess LoS path loss of $26 \mathrm{GHz}$ compared to $2.6 \mathrm{GHz}$ band in an urban area, using system level simulation [18].

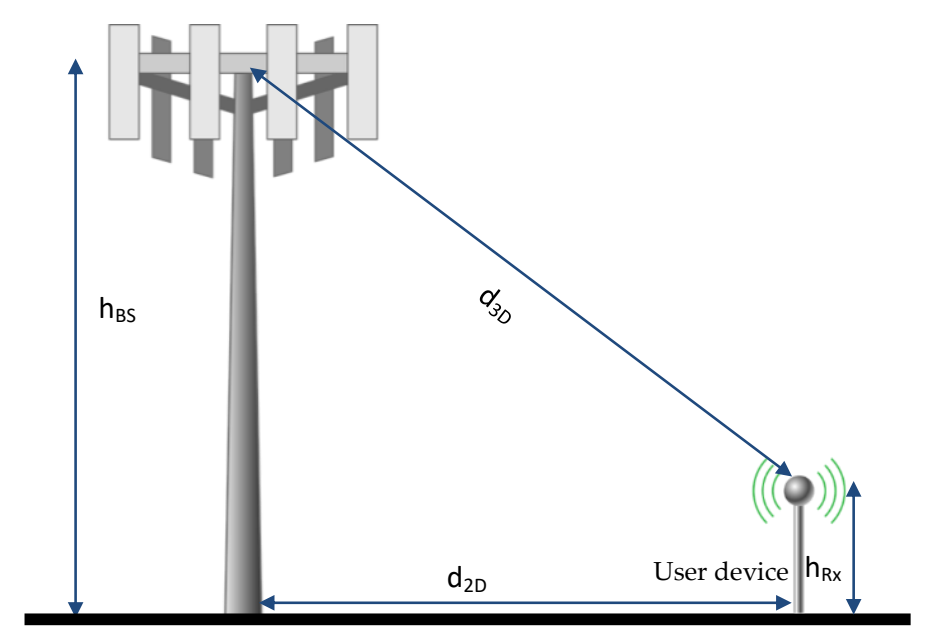

Fig.1. Path loss model representation defines d2D and d3D [19].

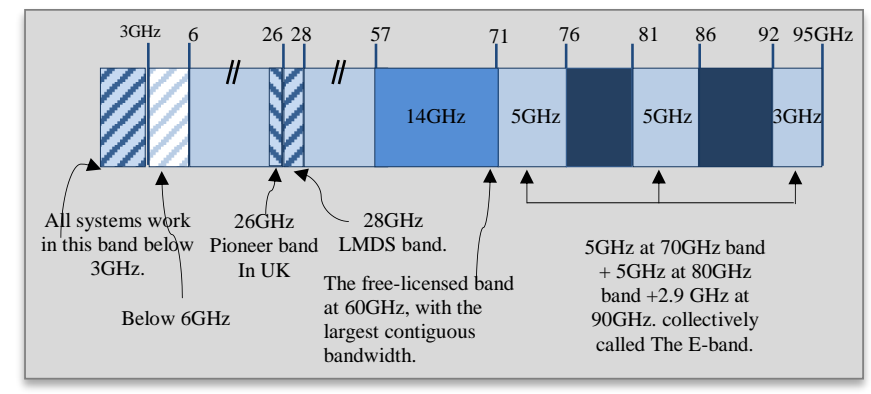

Fig.2. mm-wave candidate bandwidth for $5 \mathrm{G}$. 


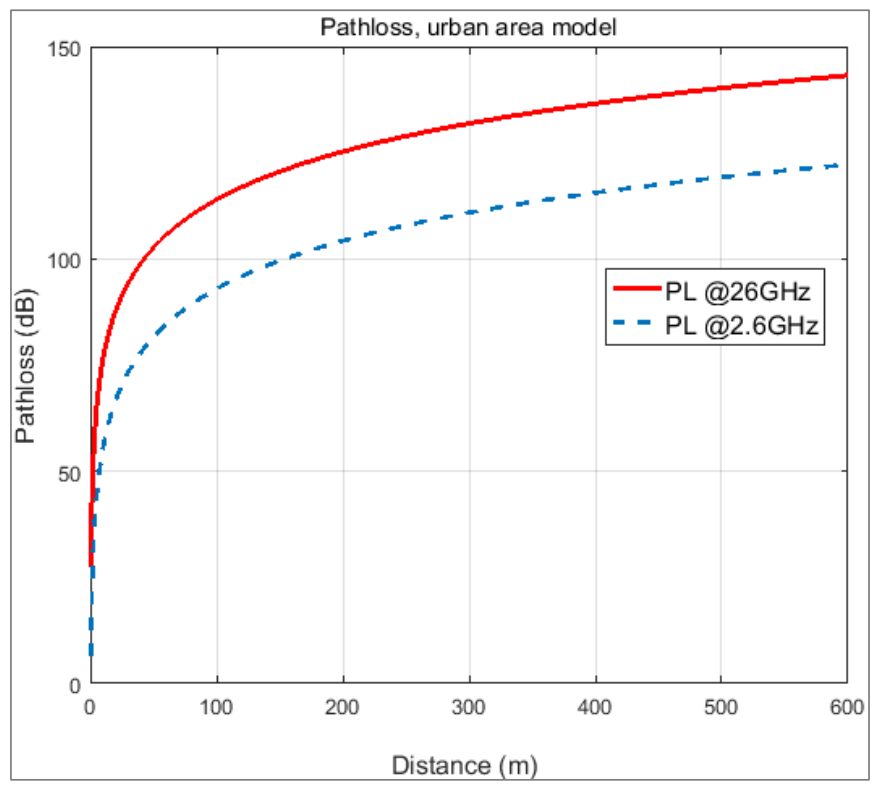

Fig.3. LoS Path loss, urban area model at 2.6 and $26 \mathrm{GHz}$.

\subsection{High Atmospheric Attenuation}

The severe attenuation at mm-wave bands is a one of the major issues that limit mm-wave propagation. This is because mm-wave energy is absorbed by oxygen molecules and water vapour in the atmosphere. The oxygen absorbs Electro-Magnetic (EM) energy at the $60 \mathrm{GHz}$ band; therefore, the $57-64 \mathrm{GHz}$ band has very high atmospheric attenuation around $15 \mathrm{~dB} / \mathrm{km}$, as shown in Fig.4. Additionally, water vapour absorbs the EM at $164-200 \mathrm{GHz}$ band with much higher loss. These bands have been excluded from the outdoor applications as the signal is extremely attenuated [4]. However, the issues with these bands have been mitigated for short link indoor communications due to the availability of very large free-licensed bandwidth at $60 \mathrm{GHz}$. Therefore, many wireless standards have been developed to operate at the $60 \mathrm{GHz}$, such as IEEE $802.15 .3 \mathrm{c}$ and IEEE 802.11 ad due to the availability of contiguous and free bandwidth [12][20].

\subsection{High Penetration Loss}

In mm-wave bands, the characteristics of building materials have a major impact on the level of the penetration loss, therefore, the building penetration loss is different from building to building, depending on the building materials used and their thickness [2]. The penetration loss of materials is calculated from the mean power transmitted through these materials [21].

The mm-wave signals have significant penetration loss through solid materials due to their short wavelengths. Severe attenuation can prevent the reception of indoor users from outdoor cells operating at mm-wave communication. Therefore, the indoor coverage can be provided by indoor mm-wave small-cells and hotspot or Wi-Fi solutions. The details of penetration losses for these frequencies are illustrated in Table 1. High levels of penetration loss consequently reduces the spectral efficiency, energy efficiency, and data throughput of mm-wave cellular networks [22]. 


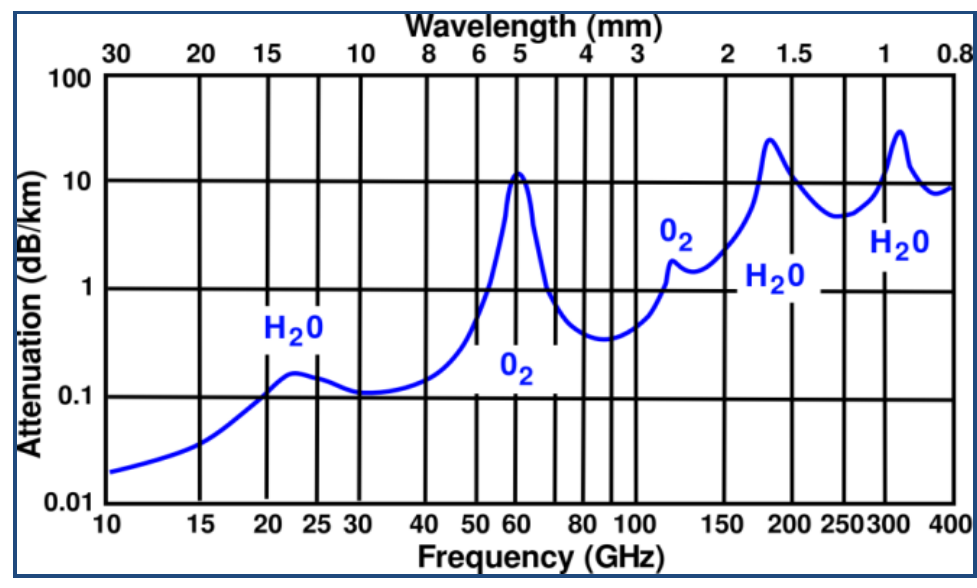

Fig.4. Average atmospheric attenuation of mm-wave [23].

Table 1 Penetration Loss at mm-wave for specific material.

\begin{tabular}{lll|l|l|l}
\hline \multirow{2}{*}{ Material } & \multicolumn{5}{c}{ Penetration Loss (dB) } \\
\cline { 3 - 6 } & Thick $(\mathrm{cm})$ & $\begin{array}{l}\text { sub 2.5GHz } \\
{[4][24][25]}\end{array}$ & $\begin{array}{l}28 \mathrm{GHz} \\
{[26][25]}\end{array}$ & $\begin{array}{l}40 \mathrm{GHz} \\
{[27]}\end{array}$ & $\begin{array}{l}60 \mathrm{GHz} \\
{[24]}\end{array}$ \\
\hline Concrete & 10 & 17.7 & 34.1 & 175 & - \\
Brick wall & 10 & - & 28.3 & 178 & - \\
Drywall & 2.5 & 5.4 & 6.8 & - & 6 \\
Whiteboard & 1.9 & 0.5 & - & - & 9.6 \\
Wood & 0.7 & 5.4 & - & 3.5 & - \\
Chip wood & 1.6 & - & - & 0.6 & - \\
Mortar & 10 & - & - & 160 & - \\
Clear Glass & 0.3 & 6.4 & 3.9 & 2.5 & 3.6 \\
Mesh Glass & 0.3 & 7.7 & - & - & 10.2 \\
Tinted glass & 0.38 & - & 24.5 & - & - \\
\hline
\end{tabular}

The penetration loss of each material in table 1 was calculated by measuring the difference in received power between LoS free space measurements with no obstructions compared with the same measurement with obstructing material between the transmitter and receiver [26].

As per table 1, very high attenuation loss for certain materials such as concrete walls can make mm-wave transmission from outdoor cells limited to outdoor users only, despite the fact that signals can transmitted inside the buildings through glass windows. The indoor solution can be provided by indoor mm-wave small-cells or mm-wave Wi-Fi [4]. While the penetration loss through solid material such as concrete walls, metal objects, and the human body can result in severe loss, they are considered as good mm-wave reflectors, which support NLoS transmission, as demonstrated in [13].

\subsection{Foliage Loss}

Foliage loss is a major mm-wave impairment, causing severe signal scattering in the presence of foliage. An empirical formula was developed in [23] by M. Marcus and B. Pattan, who represented the foliage loss by:

$$
L_{F}=0.2 f^{0.3} R^{0.6}
$$

where $L_{F}$ is the foliage loss in $\mathrm{dB}, f$ : is the carrier frequency in $\mathrm{MHz}$; $R$ : is the foliage depth in metres. This formula is applied for a foliage depth less than $400 \mathrm{~m}$ and for 20 to $95 \mathrm{GHz}$ frequencies. We plot the penetration loss against the operating frequency for different foliage depths in Fig.5. For example, the foliage loss at $28 \mathrm{GHz}$ and $73 \mathrm{GHz}$ for a penetration of 5 meters foliage is around 11 and $15 \mathrm{~dB}$, respectively, with the difference around $4 \mathrm{~dB}$. It is worth mentioning that the difference is not the same at greater foliage depths. Since the formula has a nonlinear relationship, this difference increases dramatically with foliage depth. 


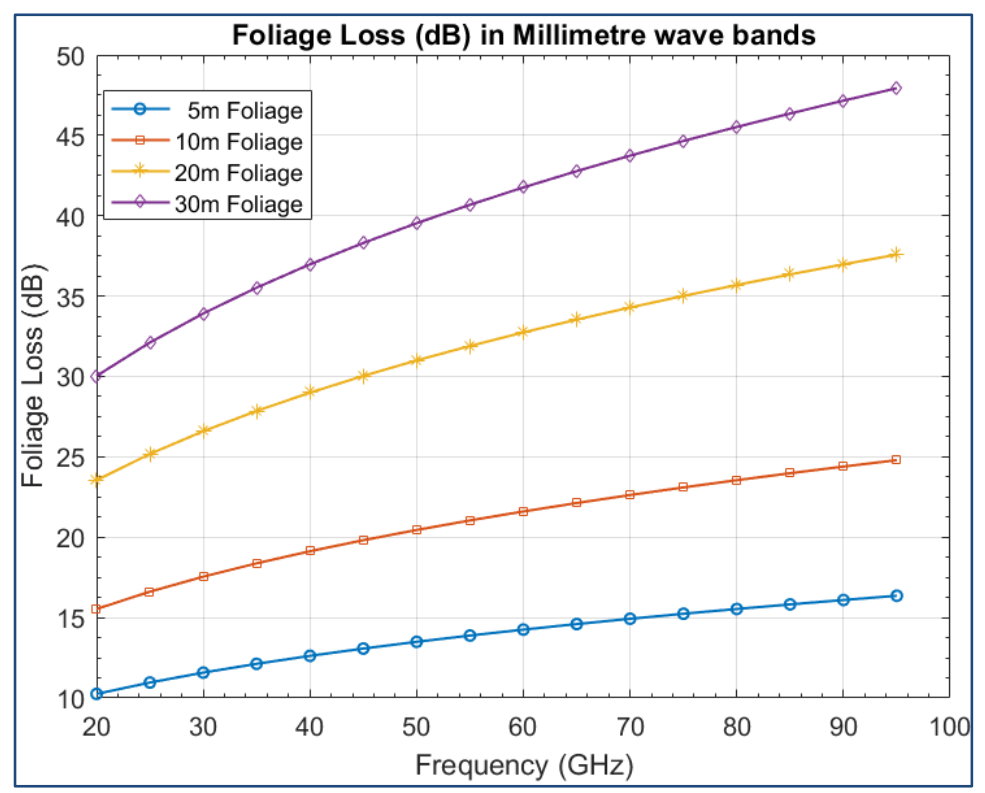

Fig.5. Foliage loss at mm-wave with different foliage depth.

\subsection{Rain Attenuation}

Rain attenuation, known as Specific attenuation $\gamma_{R}(\mathrm{~dB} / \mathrm{km})$, is obtained from the rain rate $\mathrm{R}_{\mathrm{R}}(\mathrm{mm} / \mathrm{h}) \mathrm{using}$ [28]:

$$
\gamma_{R}=k R_{R}^{\alpha} \quad d B / k m
$$

where $\gamma_{R}$ is the rain loss, $R$ is the distance between the user and the access point, and $k$ and $\alpha$ are given in ([28] Table 1) which are frequency dependent coefficients that are used for linear polarizations and horizontal paths. The values in ([28] Table 1) are sufficiently accurate for the prediction of rain attenuation up to $55 \mathrm{GHz}$, meaning that they cover a large portion of the mm-wave band, particularly the frequency of interest, $28 \mathrm{GHz}$. The coefficients $\mathrm{k}$ and $\alpha$ are determined as a function of frequency, from the following equations- derived from curve-fitting to power-law coefficients, which have been derived from scattering calculations:

$$
\begin{gathered}
\log k=\sum_{j=1}^{3}\left(a_{j} \exp \left[-\left(\frac{\log f-b_{j}}{c_{j}}\right)^{2}\right]\right)+m_{k} \log f+c_{k} \\
\alpha=\sum_{i=1}^{4}\left(a_{i} \exp \left[-\left(\frac{\log f-b_{i}}{c_{i}}\right)^{2}\right]\right)+m_{\alpha} \log f+c_{\alpha}
\end{gathered}
$$

where: $f$ : frequency $(\mathrm{GHz}), k$ : either $k_{H}$ or $k_{V}$, and $\alpha$ : either $\alpha_{H}$ or $\alpha_{V}$. Details of the frequency dependant coefficients ( $a$, $b, c, m_{k}, m_{\alpha}, c_{k}$, and $c_{\alpha}$ ) are given in tables 1,2 and 3 in [28].

Therefore, mm-wave signals can suffer significant attenuation in heavy rain as raindrops are comparable size to mmwave wavelengths and therefore cause high signal scattering. The attenuation due to rain affecting mm-waves is modelled in Matlab, where the rain attenuation over frequency is shown in Fig.6a with different levels of rainfall. For example, light rain of $2.5 \mathrm{~mm} / \mathrm{h}$ causes attenuation around $1 \mathrm{~dB} / \mathrm{km}$, while heavy rain of $150 \mathrm{~mm} / \mathrm{h}$ can jeopardize communication links at mm-wave frequencies [4][29]. Moreover, decreasing the ISD will decrease the distance between the transmitting Base Station (BS) and the receiving user, which consequently decreases the rain attenuation, as shown in Fig.6b. Here, different carrier frequencies of $28,60,72$, and $82 \mathrm{GHz}$ are compared, where the $28 \mathrm{GHz}$ frequency has the lowest figures due to the longer wavelengths compared to the raindrops. Therefore, we have introduced network densification by using dense small-cells deployment in order to minimise rain attenuation on mm-wave use by reducing the ISD among mmwave nodes. 

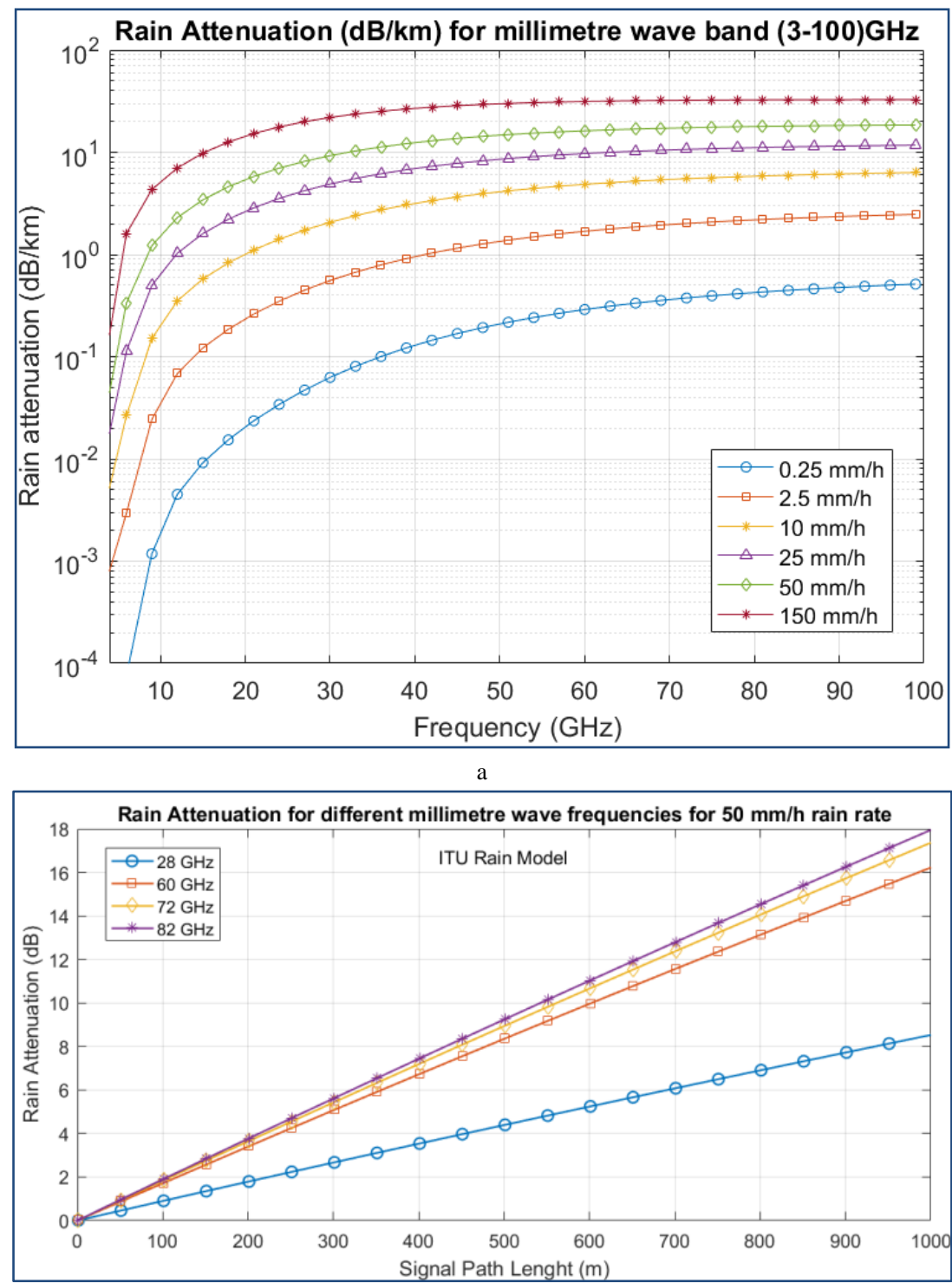

b

Fig.6. Rain Attenuation for mm-wave band, a) Rain attenuation for 3-100GHz band,

b) Rain attenuation with distance, for frequency $28,60,72,82 \mathrm{GHz}$.

These losses demonstrate how mm-wave signals can suffer significant and serious loss when they propagate in medium and high rainfall. Additionally, foliage is considered an additional serious impairment at these bands compared to the sub $6 \mathrm{GHz}$ band. Therefore, $5 \mathrm{G}$ requires radical change in the network level and component level when using mm-wave [5].

\section{Coverage and Capacity in $\mathrm{mm}$-wave}

It is widely accepted that cellular deployments can use the 26/28/38GHz [30][31][32] and the $60 / 70 \mathrm{GHz}$ bands [33][21] for coverage provision. However, in the initial deployment phase of 5G, there could be coverage problems in some areas which are covered by mm-wave nodes. Using mm-wave, the propagation will mainly depend on LoS coverage due to the weakness of reflected and diffracted signals. However, NLoS propagation can be a useful signal in certain areas especially the first Multi-Path Component (MPC). Foliage loss can however, severely attenuate mm-wave signals and can be a limiting factor in future radio network planning. Relay stations can also be used as a viable solution to improve coverage in a mm-wave $5 \mathrm{G}$ network as shown in [34][35]. The following subsections present mm-wave scenarios used for mobile networks:

\subsection{Mm-wave for Indoor Solution}

As mentioned in the previous section, the $60 \mathrm{GHz}$ band (57-64) $\mathrm{GHz}$ has been released as a free-licensed band. Due to its high atmospheric attenuation, this band can be deployed for very short distance applications such as indoor solutions in future 5G networks. Nowadays, most of the cellular traffic (around 70\%) is generated indoors at homes, offices, malls, 
and other public places [36]. Generally, indoor coverage is often poor in terms of Signal to Interference and Noise Ratio (SINR) due to the high penetration losses of outdoor-to-indoor transmission. Here, indoor users may not consume enough resources from outdoor congested nodes and experience bad Quality of Service (QoS) [37]. Therefore, indoor solution nodes would have significant advantages in offloading outdoor nodes and improving QoS for indoor users. In 5G, indoor users expect to be served by mm-wave band nodes that will provide both extremely high capacity and the necessary resources for higher data rates. One of the challenges in this context is the high penetration loss at high frequencies. Material penetration losses are higher for mm-wave than below $3 \mathrm{GHz}$. However, this issue can be addressed by minimising Tx-Rx separation. The followings are some contributions in this regard.

In [24], a measurement campaign and analysis of indoor-building at $2.5 \mathrm{GHz}$ and $60 \mathrm{GHz}$ wireless channels have been conducted. The measurements have been analysed with site-specific information for multiple materials in an indoor building to address the penetration loss for these materials. Furthermore, in [25][26], a measurement campaign has been undertaken to characterise the penetration loss at $28 \mathrm{GHz}$ for different materials and for indoor and outdoor scenarios. In [24] and [38], the penetration loss for indoor users has been measured for 60 and $73 \mathrm{GHz}$. Additionally, in [27], an outdoor penetration loss measurement campaign was conducted at $40 \mathrm{GHz}$. The above contributions lead to the conclusion that even if high penetration losses exist from outdoor nodes, this will help reduce interference between indoor and outdoor mm-wave nodes, which will eventually allow greater frequency reuse. However, a small percentage of mm-wave might transmitted into buildings through their glass-windows and wooden doors due to their lower penetration loss, which provides coverage for indoor users. Some recent contributions to the topic of indoor analysis, simulation, and measurements in mm-wave have been summarised in Table 2.

In indoor scenario simulations, when the physical environment is well-specified, and where the details of areas such as operation, layouts, materials, walls, floors and ceilings are known, software simulation tools can be employed on a very large scale of accuracy. For mm-wave, a highly directional antenna is recommended to compensate for added losses due to path loss, penetration loss and atmospheric loss. Due to the highly directional antennas adopted here, cluster analysis is necessary to determine signal reflection and possible multipath clusters for optimum signal coverage. A cluster [39] is defined as a group of multipath components (MPCs) that have similar Time of Arrival (ToA) and Directions of Departure (DoD) and Arrival (DoA). The estimated MPCs are grouped into clusters according to their ToA, DoD, and DoA. These clusters represent the area of the desired EM field, such that users can use these signals to access the network. In [39], $\mathrm{mm}$-wave multipath clustering and channel modelling campaign at $60 \mathrm{GHz}$ was conducted for indoor building to characterize MPC clustering.

The impact of environmental geometry on the clustering phenomenon of the mm-wave channel was captured by [40] using the ray tracing method; an effective simulation tool to predict the clusters location around the receiver. These results were confirmed empirically using the mm-wave channel sounding system at $60 \mathrm{GHz}$. In addition, details of clustering information for the $3.5 \mathrm{GHz}$ band can be found in [41] for wideband 3D MIMO channels in the outdoor-toindoor scenario. Having the distribution of energy around the receiver and the geometry of array antennas facilitate the design of a system with superior performance by harnessing the clusters locations and their characteristics. Such information is necessary in mm-wave indoor networks for more efficient system design. In Table 3, we have summarised some contributions to understanding of mm-wave signal clustering for indoor scenarios.

Table 2 Some contributions in mm-wave for indoor mobile access.

\begin{tabular}{llll}
\hline Ref. & Freq. $(\mathbf{G H z})$ & Method & Description \\
\hline$[42]$ & 28 & Measurements & Cluster analysis using synchronous channel sounder \\
{$[24]$} & 60 & Measurements & Penetration Loss at different material \\
{$[25]$} & $(11,28)$ & Measurements & Building penetration loss \\
{$[26]$} & 28 & Measurements & Reflection and penetration loss study \\
{$[38]$} & 72 & Measurements & Measurements for penetration loss analysis \\
{$[43]$} & 28 & Measurements & Outdoor to Indoor Coverage provisioning \\
{$[44]$} & 60 & Measurements & Empirical study on MIMO analysis for IEEE 802.15 .3 c standard \\
{$[45]$} & 60 & Measurements & Single carrier Ethernet receiver \\
{$[46]$} & 60 & Measurements & Cluster, channel model and polarisation impact \\
{$[47]$} & 15 & Measurements & Multipath and path loss study \\
{$[40]$} & 60 & Measurement \& Sim. & Cluster Analysis with directional antenna \\
{$[48]$} & 60 & Measurement \& Sim. & Cluster identification and measurements \\
{$[21]$} & $(10,30,60)$ & Simulation & Outdoor to Indoor Coverage provisioning \\
\hline
\end{tabular}




\begin{tabular}{llll}
\hline$[37]$ & $(2.6 \& 28)$ & Simulation & UDN for high capacity and cell edge improvement \\
{$[49]$} & 60 & Simulation & Spatial multiplexing gain for short-range applications \\
{$[50]$} & 300 & Simulation & Channel model proposal based on blocking probability \\
{$[51]$} & 60 & Simulation & Inter-network coordination evaluation using ray tracing \\
{$[52]$} & 60 & Simulation & mm-wave Wi-Fi Solution based on IEEE 802.11ad \\
\hline
\end{tabular}

Table 3 Some contributions on MPCs clustering at mm-wave band.

\begin{tabular}{l|c|l}
\hline \multirow{2}{*}{ Ref. } & \multicolumn{2}{|c}{ mm-wave band MPCs Clustering } \\
\cline { 2 - 3 } & Freq.(GHz) & Description \\
\hline$[41]$ & 3.5 & Comparison of clustering performance between 2D and 3D MIMO \\
{$[42]$} & 28 & Indoor measurements using synch, channel sounder and horn antennas. \\
{$[40][53]$} & 60 & Influence of geometry on the clustering phenomenon using channel sounder \\
{$[39]$} & 60 & Channel Modelling using cluster info. \\
{$[54]$} & 60 & First-order-reflection MIMO model at 60GHz carrier - indoor WLAN applications \\
{$[55]$} & 73 & Rate analysis for 3D mm-wave massive MIMO Systems. \\
\hline
\end{tabular}

\subsection{Mm-wave for Outdoor Solutions}

Mm-wave communications, with viable multi-Gbps data rates, have attracted the research attention for mm-wave as a feasible bandwidth for 5G [56]. As mentioned previously, it is anticipated that mm-wave will provide poor coverage in areas that have a low density of BSs. Therefore, the LTE systems should provide the coverage when mm-wave deployment starts. A hybrid 5G plus 4G network (multi-tier) will serve to improve network coverage, capacity, and user experience in mobile communication, as shown in Fig.7. Here, network information, control signalling, and feedback could be transmitted in the spectrum below $3 \mathrm{GHz}$, leaving the whole mm-wave spectrum available for data with faster data throughput [4][5].

However, using mm-wave in outdoor scenarios will impose many challenges due to their high path loss and amphoteric attenuation. Beamforming in-conjunction with the use of high gain/large array antennas at the transmitter and receiver end will ensure reliable links for deploying 5G networks [33]. High gain antennas can be deployed in a small physical area due to the very small wavelength in the $\mathrm{mm}$-wave band [57]. The unique propagation characteristics of mm-wave necessitates fundamental changes to the system architecture and design choices. New propagation models and new waveforms must be optimised to efficiently utilise the network resources. However, these changes will offer superior user experience through multi Gbps data rates, lower latency, and higher network capacity and connectivity. Measurement campaigns in New York and Brooklyn have shown that large contiguous bandwidths in the mm-wave band are a potential and feasible option for outdoor mobile access at $28 \mathrm{GHz}$ [58][59] and $73 \mathrm{GHz}$ [60]. Highly directional antennas are necessary here to compensate for the high losses at these bands. As shown in Fig.4 of the present paper, atmospheric attenuation will be negligible when planning the mm-wave node with ISD less than 200m [12][59]. In the case of rain attenuation shown in Fig.6, with heavy rainfall of $25 \mathrm{~mm} / \mathrm{h}$, the attenuation is about $1.4 \mathrm{~dB}$ for $28 \mathrm{GHz}, 1.6 \mathrm{~dB}$ for $38 \mathrm{GHz}$, and $2 \mathrm{~dB}$ for $73 \mathrm{GHz}$ at $200 \mathrm{~m}$ ISD. ISD $<200 \mathrm{~m}$ will be the dominant theme for future deployment. Currently, femtocells and pico-cells have a maximum ISD of 100 to $150 \mathrm{~m}$ [60].

Many experimental studies have been conducted for different frequencies in the mm-wave band. Detailed studies were conducted in the 28 and $38 \mathrm{GHz}$ bands to measure path loss, RMS delay spread, and signal coverage/outage in mm-wave outdoor channels based on steerable antenna architectures [59][15]. The authors have developed a propagation model for mm-wave and assess the feasibility of this band as a candidate band for $5 \mathrm{G}$ networks. In addition to that, as mm-wave depends highly on LoS transmission, providing Multiple Input Multiple Output (MIMO) links in a LoS environment is a challenging issue. In [61] the authors demonstrate that spatial multiplexing in a MIMO link is possible in mm-wave with potentially very high data throughput. They had achieved spatial multiplexing in LoS transmission in mm-wave. This is achieved by distributing the antennas with suitable spacing [62] in order to create independent streams with low correlation, to enable MIMO with spatial multiplexing. As a result, a considerable improvement in terms of data throughput has been achieved. More recent contributions that studied mm-wave for outdoor cellular networks are summarised in Table 4. 


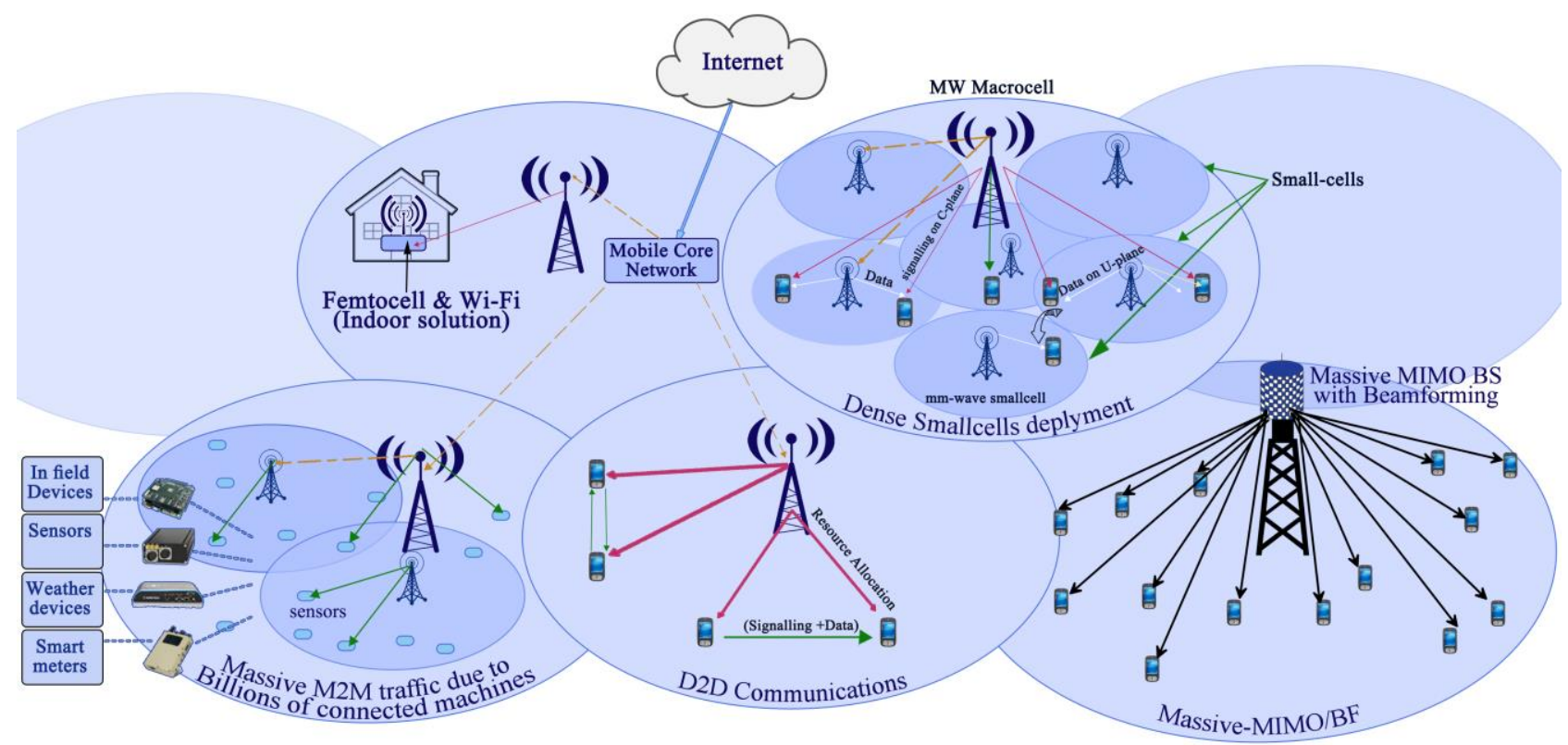

Fig.7. 5G network architecture comprises macro-cells (MW band), small-cells (mm-wave band), femto-cells and Wi-Fi (indoor solution), massive MIMO. Solid arrows represent the fronthaul, while dashed arrows represents the backhaul [5].

\subsection{Mm-wave Outdoor to Indoor}

Outdoor to Indoor (O2I) measurements are also in vital need of study to address interference affecting transmissions from outdoor nodes to indoor nodes, measure the possible indoor coverage achievable with outdoor nodes, examine the possibility of providing links to indoor wall-mounted small-cells, and accordingly to develop a suitable propagation model. O2I coverage depends on the reflection and penetration of mm-wave signals through various materials inside buildings, such as, concrete walls, glass windows, and wooden doors [63]. The measurements at 29 and $61 \mathrm{GHz}$ bands carried out in [63] were conducted to assess the feasibility of O2I coverage in mm-wave band. In [43], an O2I experimental study conducted in the $28 \mathrm{GHz}$ band is reported. This scenario makes it possible to calculate the path loss through window coated glass that encounters significant penetration loss. It found that a reliable link is feasible even with high transmission loss when a high gain antenna is used. Furthermore, in [21], two kinds of building were studied for mm-wave O2I transmission. These were an old building assumed to have $30 \%$ glass windows and $70 \%$ concrete walls, and a new building designed to have $70 \%$ Infrared Reflective Glass (IRR), which is common in the energy-saving houses, and $30 \%$ concrete wall. The test has been extended to the 10,30 , and $60 \mathrm{GHz}$ bands. The contribution of InterCell Interference (ICI) from outer nodes was included in the calculations in [37]. The work in [64] presents measurement results at $28 \mathrm{GHz}$ that investigate the effects of $\mathrm{O} 2 \mathrm{I}$ penetration loss on the propagation channel in a fixed wireless access environment. The work was conducted using real- time channel sounder and phased array antennas. The Path loss characteristics for indoor, O2I, and street "canyon" scenarios at 14.8 and $58.58 \mathrm{GHz}$ have been investigated in [65] using the 3GPP channel model described in [17]. The authors demonstrated that the path loss increases significantly with frequency, particularly in the O2I case. Therefore, outdoor mobile access in the mm- wave band is a viable solution for future networks when the planning allows very short ISD as well as highly directional antennas as has been suggested by the recent articles.

Table 4 Some contributions on mm-wave for outdoor mobile access

\begin{tabular}{llll}
\hline Ref. & Freq.(GHz) & Method & Description \\
\hline$[34]$ & $(24,60)$ & Simulation & D2D Radio propagation and channel model \\
{$[66]$} & 60 & Simulation & Soldier to soldier communications \\
{$[67]$} & 28 & Simulation & Assessment of mm-wave pico-cells network \\
{$[68]$} & 60 & Measurements & UWB path loss model \\
{$[69]$} & 38 & Measurements & Weather events on mm-wave link behaviour \\
{$[70]$} & $(38,60)$ & Measurements & LoS/NLoS outdoor link study \\
{$[71]$} & 28 & Measurements & DoA and DoD analysis \\
{$[15][72]$} & 38 & Measurements & Propagation models using beam antennas \\
\hline & & & 10
\end{tabular}




\begin{tabular}{llll}
\hline$[60]$ & 73 & Measurements & Mobile access and backhaul link study \\
{$[73]$} & 20 & Simulation & 5G Network planning with small-cells \\
{$[74][75]$} & 28 & Analysis & Coverage and rate analysis \\
{$[76]$} & 28 & Simulation & Net. planning use macro, micro, femto cell \\
{$[77]$} & 28 & Simulation & In-band wireless backhaul provisioning \\
{$[78]$} & $(60,70,80)$ & Analysis & Comparison on 5G wireless backhaul \\
{$[11]$} & 72 & Simulation & Air interface design and propagation study \\
{$[35]$} & 28 & Simulation & Coverage and capacity provisioning \\
{$[29]$} & 28 & Measurements & Analysis of climate change on mm-wave network \\
{$[79]$} & 28 & Sim. \& Measur. & On-chip antennas, arrays, propagation measurements \\
{$[80]$} & 60 & Simulation & LTE/Wi-Gig interworking \\
{$[81]$} & 73 & Simulation & Realistic outdoor urban blockage effects \\
{$[32]$} & $(38,73)$ & Simulation & Impact of rain on the coverage and outage probability \\
{$[82]$} & $(28,38,73)$ & Measurements & Omni path loss LoS and NLoS in the busy urban \\
{$[83]$} & 60 & Measurements & Service provision in the absence of LoS signal \\
\hline
\end{tabular}

\subsection{Enabling Higher Order Sectorisation}

Unlike conventional cellular network planning below $3 \mathrm{GHz}$, Higher Order Sectorisation (HOS) is a promising factor to improve the cellular network capacity and per user data rate [84][85]. This is because mm-wave uses highly directional antennas to compensate for the severe path loss and atmospheric attenuation. When using antenna arrays to provide $<20$ degree beamwidths, higher sectorisation order becomes feasible with minimal interference. The gain when high order sectorisation is applied at high carrier frequency was evaluated at $28 \mathrm{GHz}$. Table 5 shows the network model parameters used in system level simulations to show HOS, network densification, and rain attenuation modelling in the mm-wave band. Fig. 8 shows the network coverage map. The received signal power to interference plus noise ratio (SINR) of User Equipment (UE) in cellular system is:

$$
\gamma_{j}=\frac{P_{T} K_{1} 10^{\left(\sigma_{S} / 10\right) \zeta}}{d^{a_{1}}\left(\sum P_{i}+N_{U E}\right)}
$$

where: $\gamma_{j}$ is the SINR, $P_{T}$ is the total transmission power of the BS, $P_{i}$ is the interfering power of other BSs, $d$ is the distance between BS and UE, $K_{l}$ and $\alpha$ are propagation loss parameters, where:

$$
K_{1}=10^{-14.178} * 10^{-\frac{L_{p e n t}}{10}}
$$

$L_{p e n t}$ is the penetration loss.

$\alpha_{1}$ is the path loss exponent $=2.3$ for $\operatorname{LoS}[86]$ and $=3.86$ for NLoS transmission at $28 \mathrm{GHz}$ [12].

$N_{U E}$ is the thermal noise, $\zeta$ is the fading random variable, and $\sigma_{S F}$ is the shadow fading standard deviation in $\mathrm{dB}$.

The per user data throughput can increase according to the fundamental capacity equation defined by [87]:

$$
T P_{j}=\sum_{m=0}^{m_{i, j}}\left(B / n_{x}\right) \log _{2}\left(1+\gamma_{i, j}\right)
$$

where $T P_{j}$ is the throughput of a $\mathrm{UE}_{\mathrm{j}}, \mathrm{m}$ is the number of parallel streams between $\mathrm{BS} \mathrm{i}$ and $\mathrm{UE} \mathrm{j}, B$ is the system bandwidth, $n_{x}$ is the loading factor, which indicates the number of UEs served by a sector, and $\gamma_{i, j}$ is the SINR. 


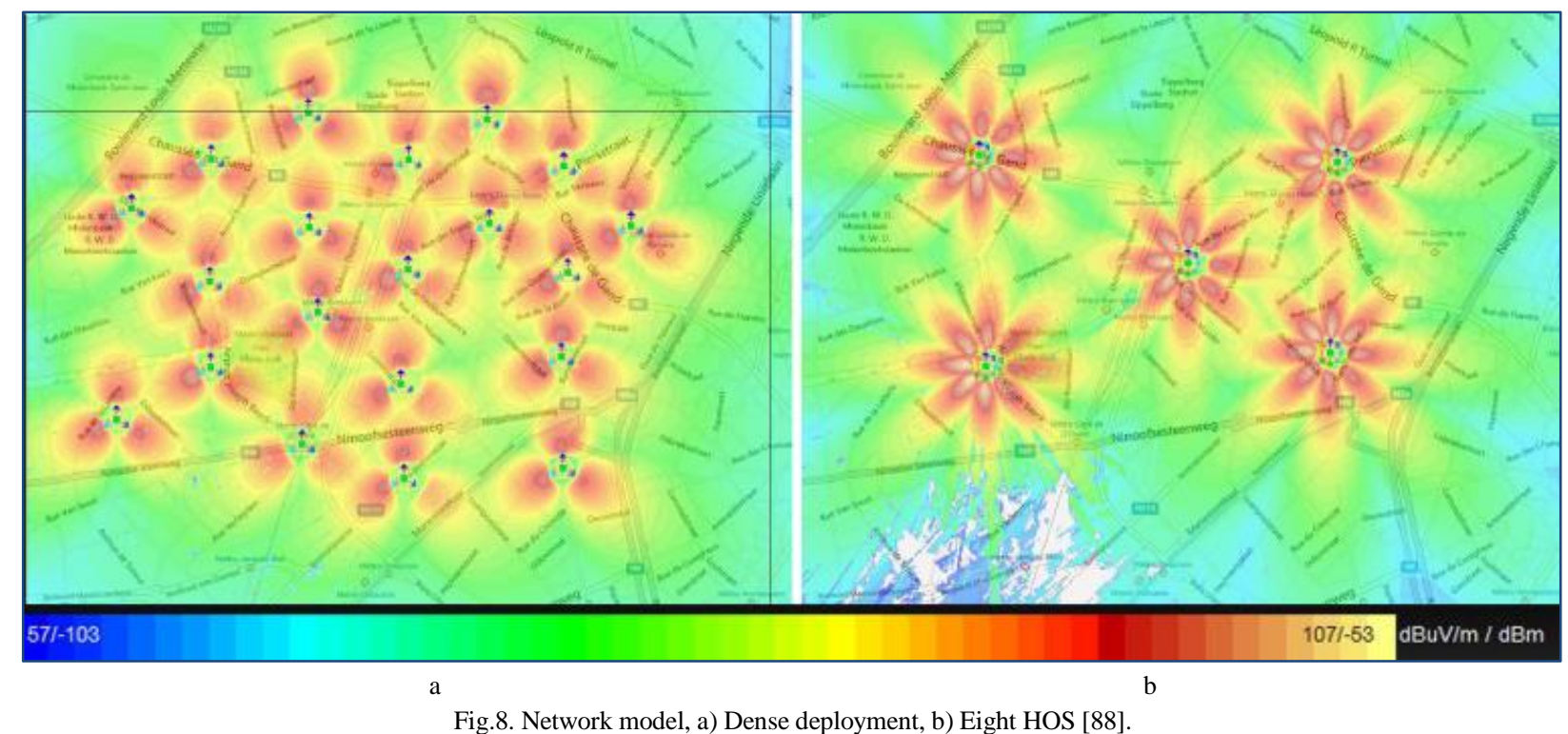

Table 5 Network Modelling Parameters for System Level Simulations

\begin{tabular}{llll}
\hline Parameter & Value & Parameter & Value \\
\hline Carrier frequency & $28 \mathrm{GHz}$ & Scheduler & Proportional Fair \\
Bandwidth & $500 \mathrm{MHz}$ & Rain (ITU-R 838 model) & $(0.25-150)$ mm/h rainfall \\
Tx Power & $10 \mathrm{~W}$ & Traffic model & Full buffer \\
Tx antenna gain & $15 \mathrm{~dB}$ & Data channels & $70 \%$ (resources) \\
Rx antenna gain & $3 \mathrm{~dB}$ & Control channels & $30 \%$ (overhead) \\
Tx pattern & $60^{\circ}(3,4,6)$ sectors, $20^{\circ}(8,10)$ sectors & Region of interest (ROI) & $600 \times 600 \mathrm{~m}$ urban \\
Tx height & $10 \mathrm{~m}$ & Noise Density & $-174 \mathrm{dBm} / \mathrm{Hz}$ \\
Rx height & $1.5 \mathrm{~m}$ & \#no. of BS & 5,10, and 20 \\
Modulation & Adaptive & HOS order & $3,4,6,8$, and 10 \\
UEs & $600($ random, outdoor) & ISD & 600,400, and $200 \mathrm{~m}$ \\
\hline
\end{tabular}

As indicated by Equation (9), the system bandwidth B can be increased by using a wider bandwidth at the mm-wave band. This increase will directly increase the data throughput (TP) due to the direct proportional relationship. The loading factor (n) can be decreased by HOS as well as higher network densification. The number of spatial streams (m) is given by $\min (\mathrm{M}, \mathrm{Nr})$ where $\mathrm{M}$ and $\mathrm{Nr}$ are the number of antennas at the transmitter and receiver, respectively. It is important to mention that as HOS order increases, ICI from surrounding cells increases consequently, which will impact the coverage probability as shown in Fig.9. Therefore, at HOS of $10^{\text {th }}$ order, the number of UEs receiving fair SINR will be lower due to increased interference, which affects the LoS and NLoS coverage probability.

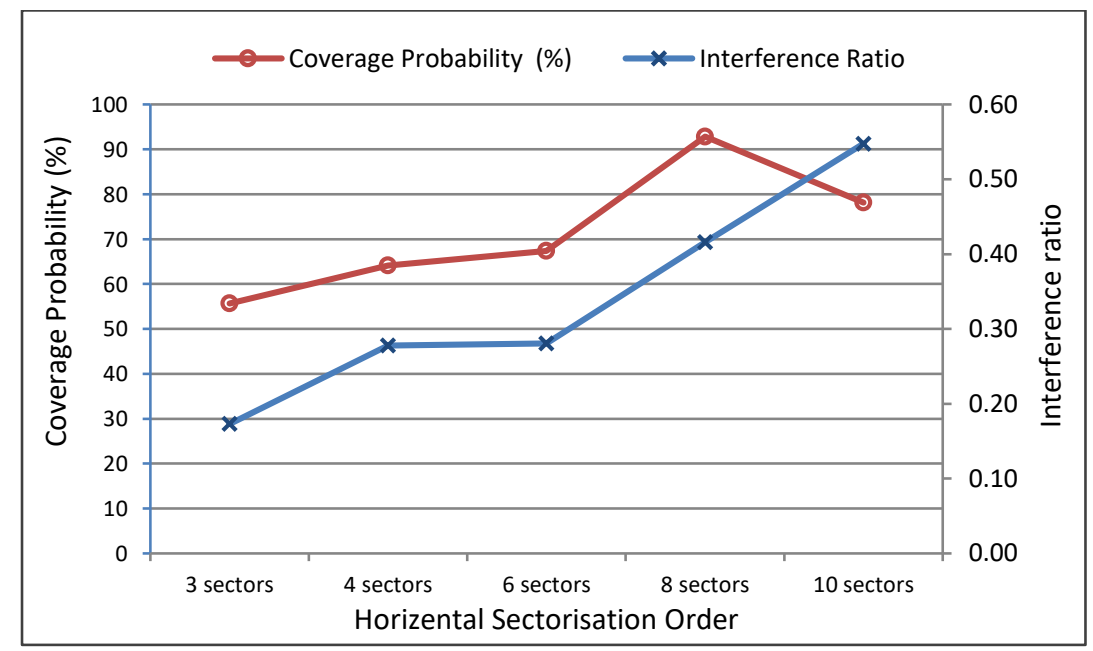

Fig.9. BS coverage probability vs. interference in multiple site topologies [89].

As shown in Fig.10, with $1 \mathrm{GHz}$ bandwidth, the average BS capacity can reach very high values. This configuration will help provide the "massive capacity" requirement of the future $5 \mathrm{G}$ network. However, the huge traffic generated by these 
nodes will need to be carried by high capacity backhaul links into the core network. Current microwave links will not be capable of handling this huge amount of traffic due to their limited capacity. This subject will be discussed in the backhaul link section of the present paper. These scenarios have been simulated using the ICS Designer tool by ATDI [90] at $28 \mathrm{GHz}$ band.

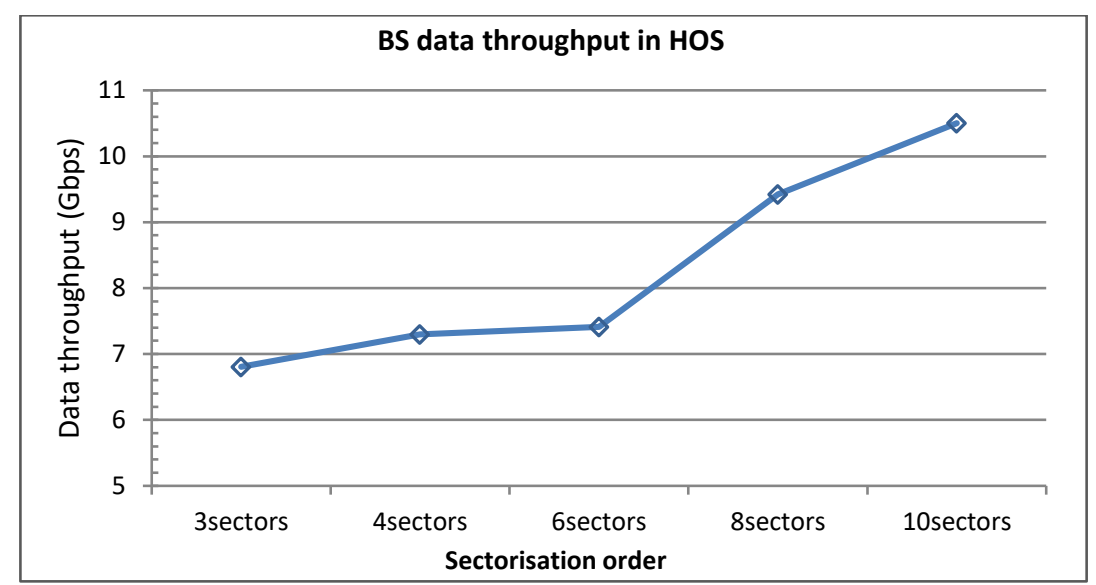

Fig.10. BS data throughput vs. different HOS order [89].

One of the advantages of using HOS in mobile network is that it improve the beamsteering efficiency [89]. For example, the BS steers its beams to their corresponding users for maximum signal reception. As the steering angle increases, the steering loss increases proportionally. In a default three sectors site, the steering should cover the sector beamwidth of $120^{\circ}$, and for eight order HOS, for example, steering requirements decrease to $45^{\circ}$. As illustrated in Fig. 11 , HOS can significantly improve beamsteering efficiency.

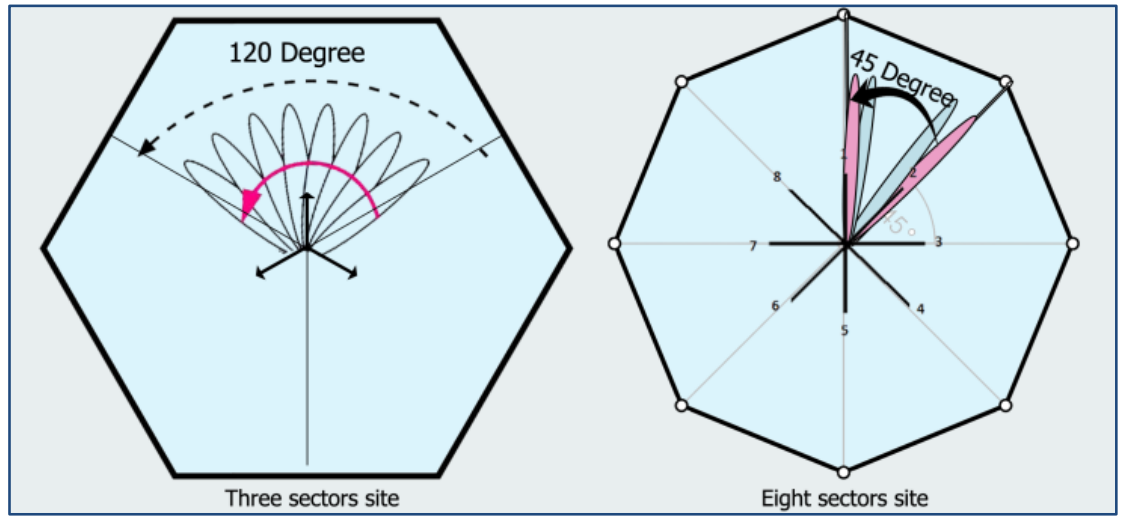

Fig.11. HOS impact on beamsteering, left is a three sectored site and right is an eight sectored site [89].

Fig.12 shows the Reference Signal Received Power (RSRP) in dBm, with and without the consideration of a heavy rainfall of $50 \mathrm{~mm} / \mathrm{h}$ with different network architectures. Scenario A has $600 \mathrm{~m}$ ISD, Scenario B has $400 \mathrm{~m}$ ISD, while Scenario $\mathrm{C}$ has $200 \mathrm{~m}$ ISD. Another scenario has been applied to $\mathrm{C}$ where antenna tilts have been optimised to provide better coverage. As per this figure, Scenario C performs better due to the short distance between the BS and UE, and therefore has increased the mm-wave link reliability in a heavy rain environment [88]. Nevertheless, an emergency MW network must be available in order to provide network service as heavy rainfall can jeopardise mm-wave communications. 


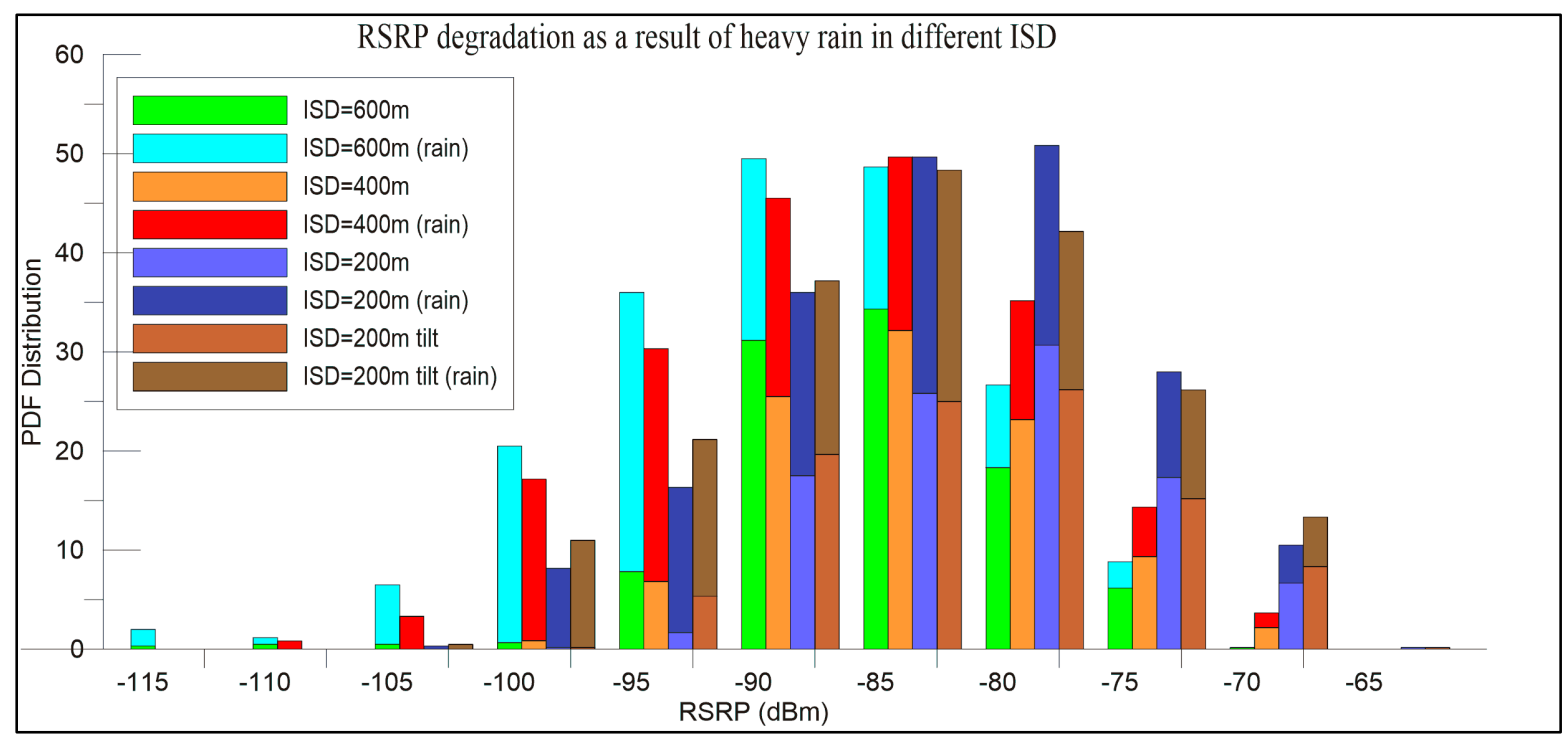

Fig.12. RSRP (dBm) degradation as a result of heavy rainfall of $50 \mathrm{~mm} / \mathrm{h}$ for different ISD [88].

\subsection{Distributed BS in $\mathrm{mm}$-wave}

In the Radio Access Network (RAN), a mobile networks architecture is modelled with a single BS, which provides cellular coverage and resources exchange to the users [91]. The default implementation is a three sectors solution, where the BS is transmitting in three directions using directional antennas. The BS antennas are co-located at the BS site, and hence the term Co-located BS (CBS) architecture. An alternative architecture is the distributed BS (DBS) architecture shown in Fig.13 [62]. The DBS network architecture has the advantage of increasing the signal power at the cell edge with Remote Radio Heads (RRH) [19]. The first DBS network investigation in using the mm-wave appeared in [92], where the author has shown the importance of using remote antennas to minimise the shadow fading of cellular networks in the local multipoint distributed service (LMDS) band. DBS splits the BS into two parts: the base band unit (BBU) part located at the centre and the RRHs part that are located remotely from their BBUs. In this scheme, the RRHs are connected with a high speed fibre link to the BBUs. Fibre links are used to power the RRHs as well as to carry the signalling.

Another approach is proposed in [93][94], where the authors suggest a network with a very large number of distributed APs that simultaneously serve a much smaller number of users. There are no cell boundaries and hence the name "CellFree Massive MIMO”. This approach has the advantage of higher data rates, with better coverage probability and energy efficiency compared to a small-cell system approach. Furthermore, the distributed nature of APs average out small-scale fading so that performance depends only on large-scale fading. Therefore, this system is robust to shadow fading correlation.

The concept of DBS with distributed RRHs is adopted in order to sidestep the lack of coverage and high path loss that characterise mm-wave communications. DBS has the potential of better QoS provision because the access points (RRHs) are now closer to the users [19]. The gain achieved by a DBS architecture is increased by increasing of the number of deployed RRHs, until a number is reached where the gain will be negligible with additional RRHs [95]. This limit is shown to be a four RRHs for uniform RRH deployment [96] and seven for random deployment [97]. 


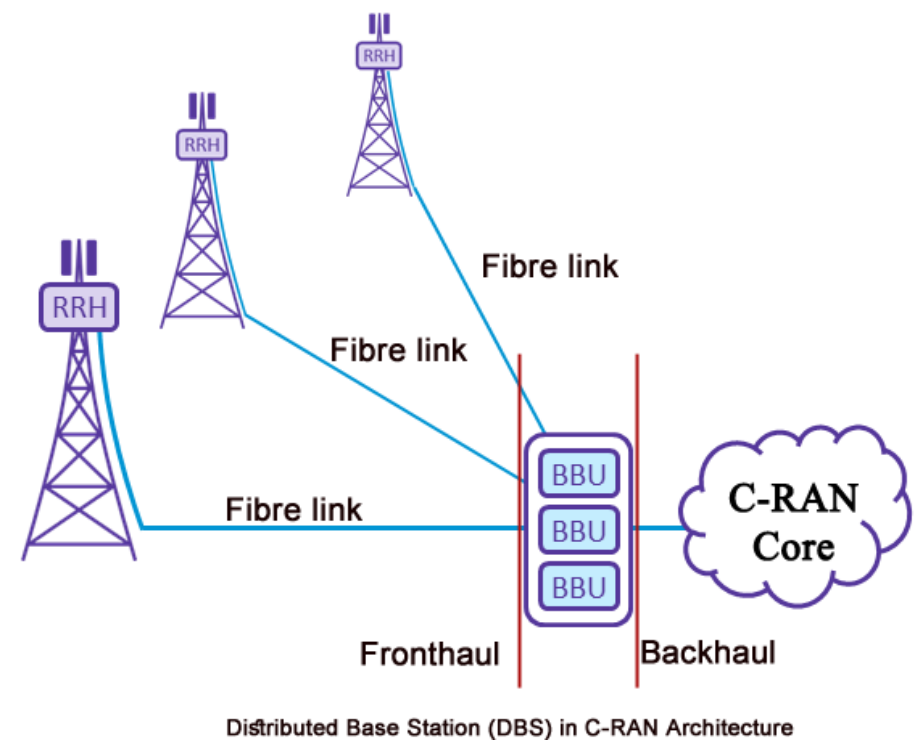

Fig.13. DBS architecture in C-RAN network.

The DBS network model is clarified in Fig.14. It consists of mm-wave BBUs and remotely distributed RRHs. The RRHs provide the connectivity to a group of UEs, symbolised by red dots. The RRHs are connected to their BBUs by a high bandwidth fibre link called fronthaul. The ISD among BBUs is $150 \mathrm{~m}$, and RRHs have been mounted at a $10 \mathrm{~m}$ height, $50 \mathrm{~m}$ apart from their corresponding BBU's. Fig. 15 presents the SINR mapping of both CBS and DBS networks with two RRHs per cell. As shown in this figure, SINR is improved in the regions where RRHs are used. The RRH system design includes transceivers, duplexers, analogue to digital conversion (ADC), filtering processes, and a Power Amplifier (PA) stage. The DBS network architecture leads to a new paradigm for 5G UDN deployment, by making the next generation network architecture efficient, flexible, and scalable.

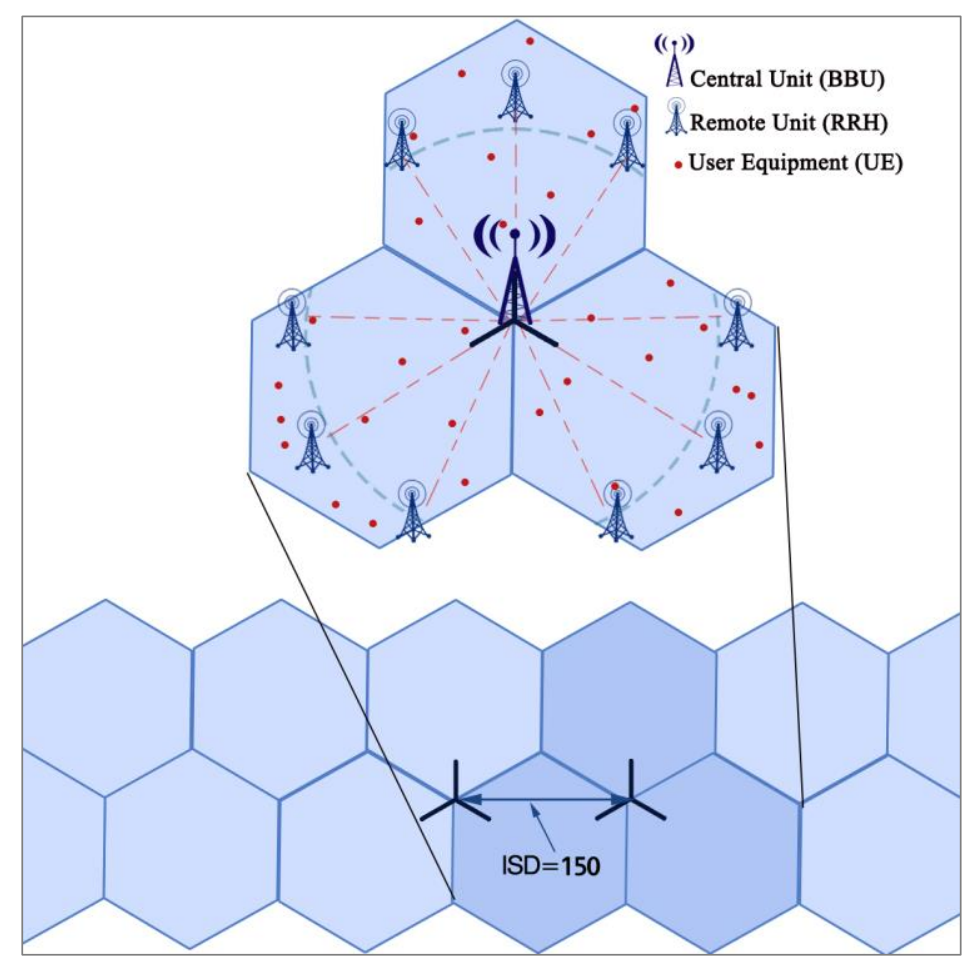

Fig.14. DBS network comprises the central node with 3 BBUs, distributed RRHs, and UEs symbolised by red dots. Whereas the dashed sky-blue arch is a radial distribution line of RRHs, and the dashed red lines refer to the fronthaul. 

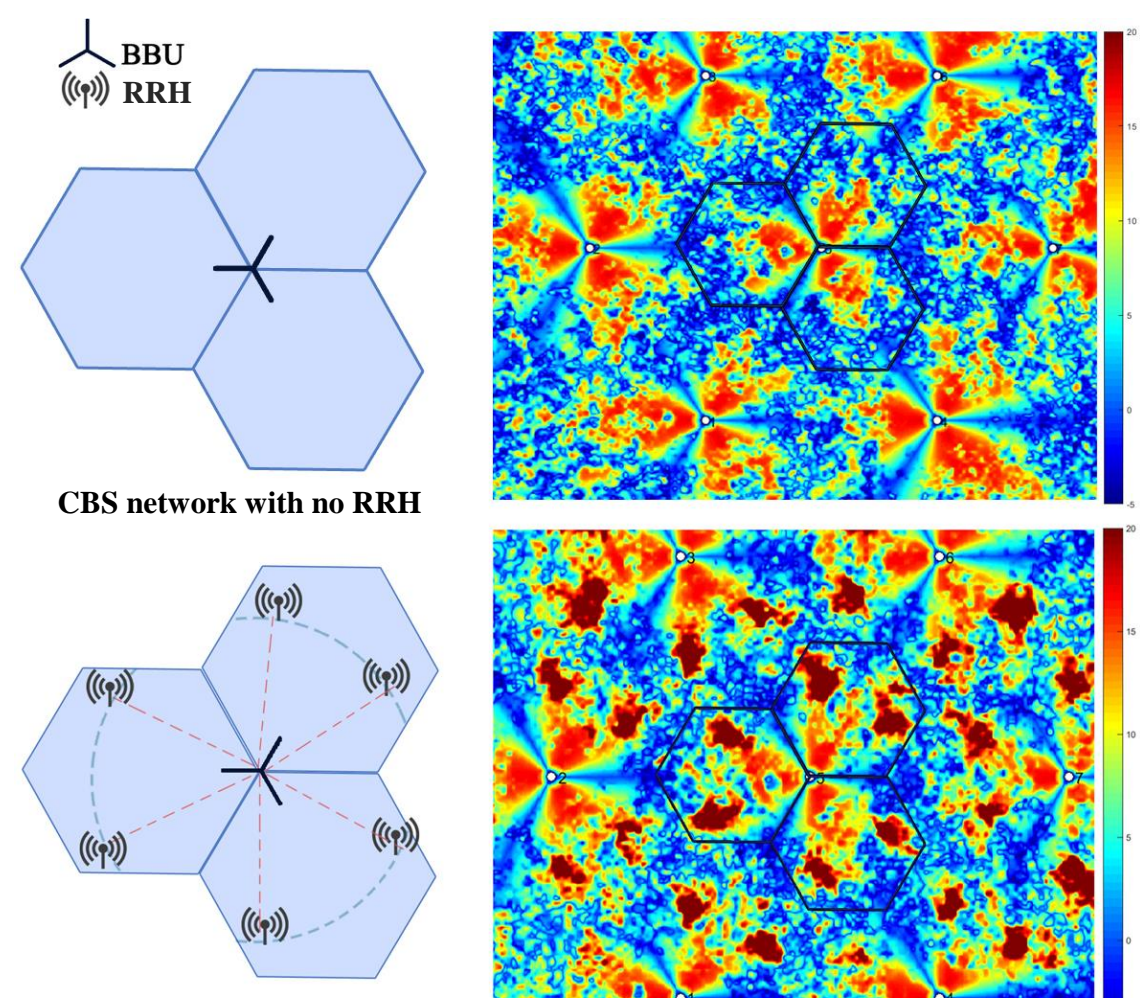

DBS network with 2RRH/BBU

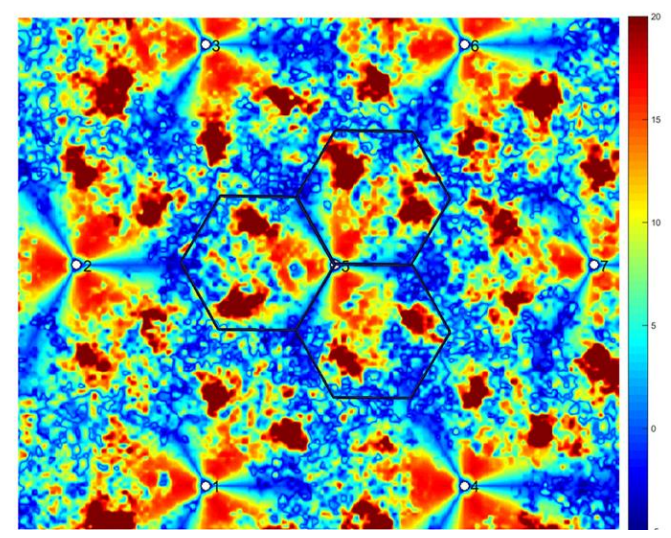

Fig.15 SINR mapping, path loss plus shadow fading map, with/out RRHs.

In this work, up to $500 \mathrm{MHz}$ bandwidth, centred at the $26 \mathrm{GHz}$ pioneer band has been used. This band has been released by Ofcom for $5 \mathrm{G}$ deployment in the UK, with a very wide bandwidth from 24.25 to $27.5 \mathrm{GHz}$ for high data rate demand areas [9]. A system level simulation has been conducted to demonstrate the advantage of using a DBS network over a CBS network. With the same number of antennas and number of UEs, the average UE data throughput is shown to be significantly improved as shown in fig.16.

Another realisation has been carried out to show the improvement of increasing the system bandwidth of 5G network, with a CBS and a DBS network architecture. A significant gain is shown in Fig.17 as the system bandwidth is increased, with the most improvement occurring with the DBS network architecture, where three RRHs have been used for each cell. With an average cell performance at $500 \mathrm{MHz}$ bandwidth, there is a $29.45 \%$ gain in average data throughput in the DBS over a CBS network.

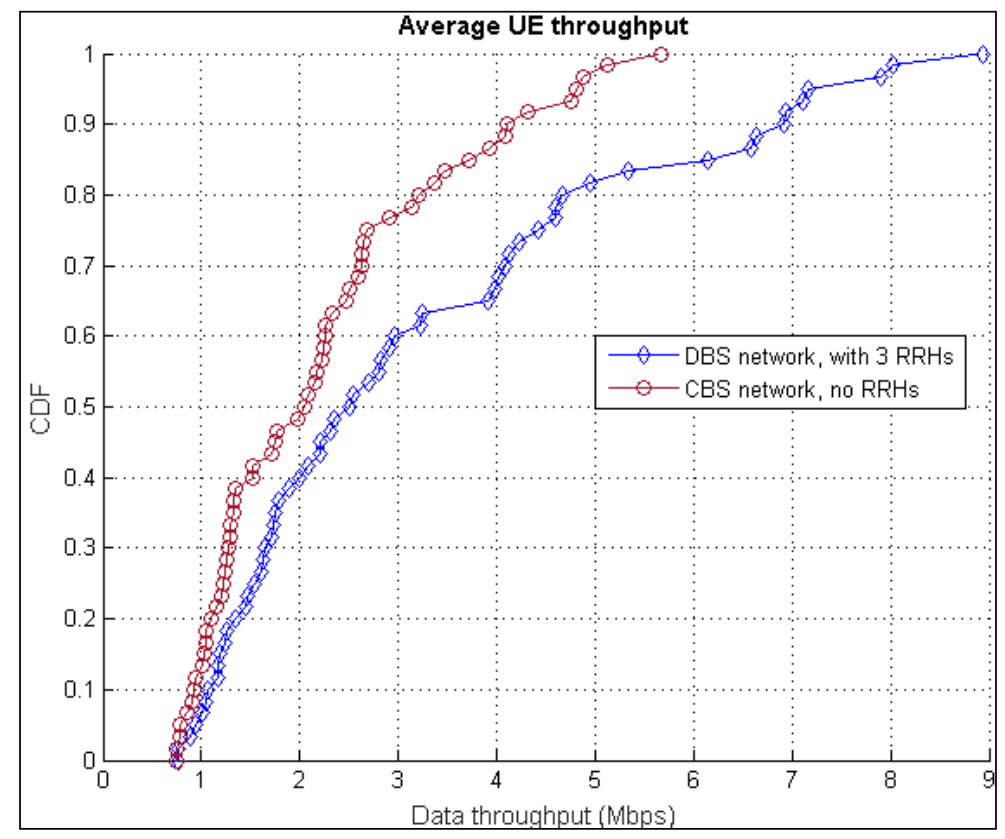


Fig.16. Average UE throughput in CBS and DBS networks [19].

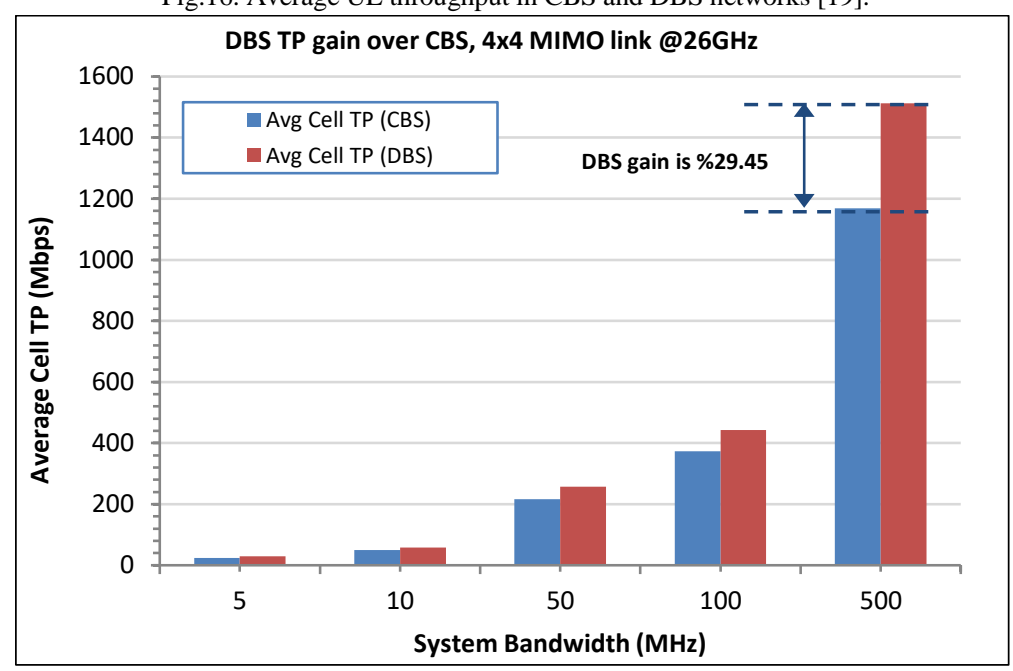

Fig.17. Data throughput vs. system bandwidth in CBS/DBS network.

\subsection{Mm-wave Massive MIMO}

Massive MIMO or large scale MIMO is the principle when a mobile network is equipped with a large number of antennas at the BS side. These "massive" antennas can accommodate multiple co-channel users simultaneously. The deployment of a massive MIMO can significantly improve the spectral and energy efficiency of the mobile network, which can help meet the technical requirements of the 5G network [5]. Furthermore, a higher number of antennas can provide greater freedom in interference coordination. However, the deployment should take into account some practical limitations of m-MIMO such as large channel estimation, pilot contamination and control signalling overhead to support large numbers of narrow beams [85].

- $\quad$ Pilot Contamination: When the BS wants to receive the channel responses of its corresponding UE, the UEs send pilot signals in the uplink. Due to the massive antennas, these pilot signals are corrupted by ICI from these interferers when received by the BS. Massive MIMO will encounter more pilot contamination with a higher number of antennas. Research activities are essential to achieve pilot orthogonality without consuming network resources [98]. Pilot contamination is generally associated with multi-cell MIMO. However, pilot contamination can also occur in a single-cell scenario, when the number of UEs are larger than the number of pilot sequences, which causes considerable performance degradation [99].

- Channel Estimation: Massive numbers of antennas used at the BS side need high accuracy CSI estimation, which is a major issue because of the resultant enormous streams and the computational complexity. Currently, only TDD mode is considered for massive MIMO because the cost of channel estimation and feedback is prohibitively expensive. The CSI acquisition is very challenging in massive MIMO, due to the high dimensionality of channel matrices, the pilot contamination in the uplink, substantial downlink training overhead, and very high computational complexity [100]. A more sophisticated algorithm is necessary to enable accurate channel estimation and to reduce signalling overhead while keeping the system latency to minimum.

- $\quad$ Large infrastructure: Massive MIMO has a very large physical architecture [101] due to the massive space required to accommodate the "massive" number of antennas. Therefore, conflicts by public and building owners will arise due to potential health issues arising from exposure to high energy EM signals. In addition, towers with larger physical architectures create extra confrontation and further technical challenges. However, a successful way to potentially address the issue of massive MIMO physical size is by using a short wavelength carrier such as in the mm-wave band. Thus, a feasible array structure will become possible due to the very short wavelength that allows a great number of antennas to be packed into a small physical area. 
Mobile systems operate in two transmission modes. In Frequency Division Duplex (FDD), the BS and UE transmit and receive simultaneously using different carrier frequencies. The bandwidths in the uplink and downlink are usually the same. This makes FDD suitable for voice communications, where both uplink and downlink transmissions have similar data rates. On the other hand, in Time Division Duplex (TDD), BS and UE transmit and receive on the same frequency but at different time slots [102].

Generally, FDD is employed in most mobile networks, but in massive MIMO, channel estimation becomes a challenging issue because the MIMO channel requires significant overhead, which increases as the number of MIMO antennas increases. This overhead can considerably limit the performance of massive MIMO [103]. BSs can estimate the Channel State Information (CSI) for downlinks based on uplink transmission training signals using channel reciprocity in TDD [104]. Therefore, UEs CSI feedback is not necessary, which makes massive MIMO feasible and tangible [105]. However, if the pilot sequences implemented in different cells are not orthogonal, pilot contamination will limit the achievable data rates in massive MIMO [106]. TDD is considered a more favourable duplexing option over FDD in the 5G UDN environment because spectrum flexibility is a more important factor [107], and TDD can reduce the training overhead in cellular network [108].

In massive MIMO, uplink pilots are used to estimate channel vectors in a TDD mode, where UEs send orthogonal pilot training sequences to the BS for CSI acquisition. The downlink channel is estimated at the BS by channel reciprocity [109]. Hence, an uplink training sequence requires resources proportional to the number of UEs. On the other hand, the FDD mode requires downlink pilots for CSI acquisition, where UEs estimate the channel response based on the received pilot and then feedback a quantized version to the BS. This process requires a substantial feedback overhead that increases linearly with the number of antennas. Therefore, FDD mode with downlink pilots is not a feasible option in massive MIMO [99]. A recent testbed proposed by Samsung R\&D operating in the $28 \mathrm{GHz}$ band was built with a bandwidth of $800 \mathrm{MHz}$ using TDD mode. The testbed has achieved a data throughput of 7.5 Gbps [110]. Nevertheless, several possible ways are proposed to enable massive MIMO in FDD mode. One way is to design efficient precoding methods based on partial CSI. Another way is to use the principle of compressed sensing to reduce the FDD feedback signalling overhead [111].

In order for the receiver to know the channel matrix, the transmitter has to send the orthogonal pilots. The sample duration of the pilot sequences should be greater than or equal to the number of transmit antennas, $\tau_{\mathrm{d}} \geq \mathrm{M}$. Uplink transmission requires the BS to learn the uplink channel matrix which requires uplink pilots of $\tau_{\mathrm{u}} \geq \mathrm{K}$. Hence the total pilot time for a complete TDD or FDD system, is [112]:

$$
\tau_{d}+\tau_{u} \geq M+K
$$

The FDD system requires relatively more time for pilot training than the TDD system. In FDD, the BS antennas (M) transmit pilots with $\tau_{\mathrm{d}} \geq \mathrm{M}$. The $\mathrm{K}$ users then transmit M pilots in the uplink, which require extra $\tau_{\mathrm{d}}$. Finally, the K users need to transmit pilot sequences with $\tau_{\mathrm{u}} \geq \mathrm{K}$. Therefore, the total training requires:

$$
2 \tau_{d}+\tau_{u} \geq 2 M+K
$$

compared with only $\tau_{\mathrm{u}} \geq \mathrm{K}$ channel for the TDD system.

However, due to the limited coherence time, the pilot training of the users in neighbouring cells may no longer be orthogonal to those within the cell, leading to a pilot contamination problem [111]. The use of mm-wave band yields higher Doppler shifts for a given user speed, and therefore shorter coherence times. This shorter coherence time at mmwave is compensated for by the lower mobility and hence the higher coherence bandwidth due to its operation in small cells. The short wavelength of mm-wave is attractive for the design of massive MIMO as the physical dimensions architecture is reduced [113].

\subsection{Beamforming in $\mathrm{mm}$-wave}

Beamforming is the concentration of power in a thin beam in order to increase the wanted signal power and efficiently suppress the unwanted signals (interference). Beamforming and massive-MIMO are the key enablers of a 5G 
system, as they can dramatically improve the SINR, which can lead to better network performance and higher data throughput at the cell-edge.

Beamforming processing can be applied in digital and in analogue baseband. In Digital beamforming, multiple spatial streams can be transmitted through multiple RF chains. However, implementing a large number of RF chains for all antennas can be prohibitively expensive due to the larger number of antennas in a mm-wave MIMO array. Here each antenna branch requires a Power Amplifier (PA) stage, Digital-to-Analogue-Conversion (DAC) and an Analogue-toDigital-Conversion (ADC) stage. These are the most expensive parts of the transceiver [98][114] and the IFFT/FFT stage, making such a design very expensive to implement. Furthermore, higher power consumption and increased complexity of signal processing in the front-end transceivers will be difficult due to hardware constraints, PA nonlinearities, and cost when using mm-wave [115][116]. On the other hand, a simpler and cheaper approach can be achieved with an Analogue BF that utilises only a single RF chain, PA, and DAC stage. This approach performs BF processing on the transmitted signal through all antennas. In this case, only a single beam can be generated. Analogue beamforming has very high efficiency with very simple and cheap architecture.Analogue beamforming is supported in the next generation multi-Gbps Wi-Fi IEEE 802.11ad standard operating in the 60GHz band [117].

Generally, digital beamforming provides a higher Degree of Freedom (DoF) to manipulate transmitter and receiver signals for improved performance at a higher cost and complexity due to separate FFT/IFFT stages, DAC/ADC per each RF chain, and the huge cost of DAC/ADC [118]. On the other hand, analogue beamforming is simple and effective in providing high beamforming gain from large number of antennas, but with less flexibility compared to digital beamforming, as shown in Fig.18a,b. Therefore, a trade-off [56] between performance/simplicity in analogue beamforming and flexibility/DoF in digital beamforming motivates the topic of Hybrid beamforming architectures, particularly in massive MIMO, where a large number of antennas require a large number of RF chains [119]. In hybrid beamforming analogue processing is performed over a group of RF chains in each transceiver, and then digital processing is performed over all transceivers [120]. This architecture significantly reduces the number of $R F$ chains $\left(\mathrm{N}_{t}\right)$ and DACs/ADCs required compared with the number of transmit antennas $\left(\mathrm{N}_{\mathrm{T}}\right)$, as shown in Fig.18c, in order to produce low cost massive MIMO systems [88][98][114].

In a UDN environment, combining analogue beamforming and digital precoding in hybrid beamforming is a more feasible solution [119]. In this scheme, the beamforming weighting algorithms are executed at the RF side (before the PA stage), where groups of antennas elements are stacked and supplied by a DAC. This scheme significantly reduces the number of RF chains, DAC stages, and implementation complexity, and maintains a reasonable number of beams. Additionally, UDN can produce significant interference that diminishes the gain of network densification, and therefore, needs to be addressed. In this context, hybrid beamforming in a UDN environment is a feasible option that can mitigate this interference, as proposed in [121]. Hybrid beamforming is considered the most appropriate architecture for wideband frequency-selective mm-wave channels [122].

In [123], a new iterative space-time receiver structure for mm-wave multi-user massive MIMO has been designed. The authors propose a low complexity UE with analogue random precoding and a single RF chain. The system can efficiently reduce multi-user interference while improving the performance closer to the digital beamforming schemes. A criterion to find the best partitioning of the massive MIMO antennas over the RF chains in hybrid beamforming has been proposed by [124]. Based on this criterion, a near-optimal solution for fully/partially connected hybrid beamforming [122] has been designed with mm-wave OFDM systems.

Fig.18c depicts a hybrid analogue-digital beamforming architecture for mm-wave communication. In this architecture, beamforming processing divides the processing into baseband precoding and RF precoding. The target of hybrid beamforming is to provide data multiplexing with reduced power consumptions and costs (by reducing the DAC/ADC stages). The low-cost phase shift network is used to decrease the number of required DAC and RF chains before digital baseband beamforming is employed [109]. Phase shifters in RF precoding are controlled digitally to form narrow beams and to enable analogue beam-steering. Whereas the digital domain processing performs actual precoding processing at the RF frontend according to the implemented precoding/combining schemes such as Zero Forcing (ZF), and Minimum Mean Square Error (MMSE) [125]. 


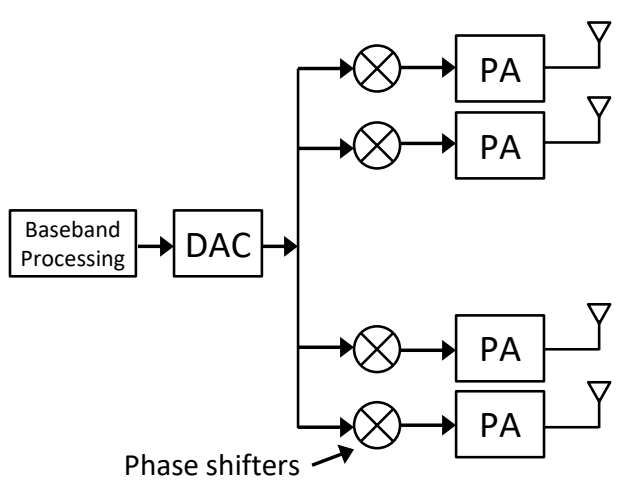

(a)

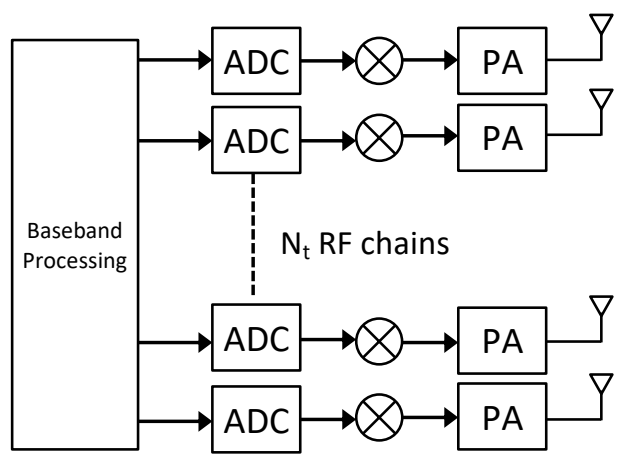

(b)

RF Precoding /phase shifter network

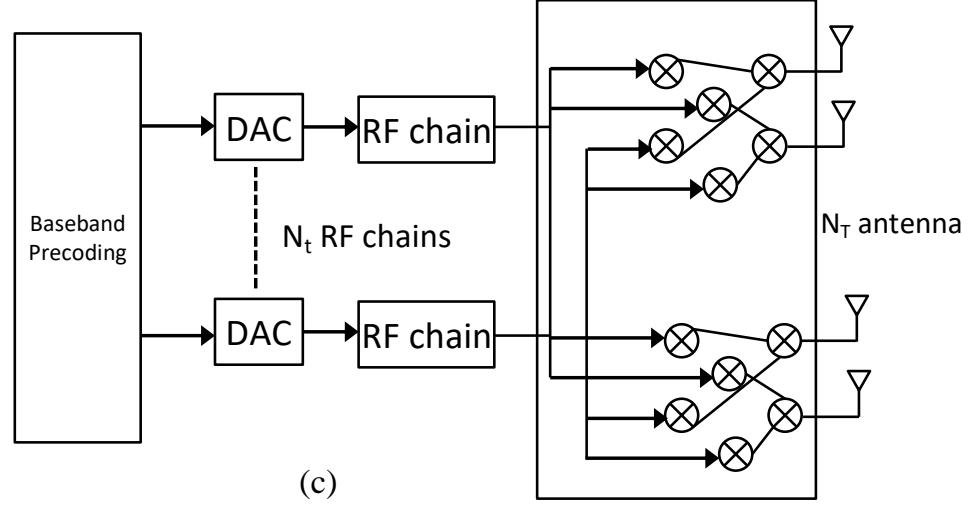

Fig.18. Beamforming options in mm-wave, (a) Analogue beamforming, (b) Digital beamforming, and (c) Hybrid analogue-digital beamforming

ZF beamforming used in CLSM achieve antenna beamforming by nullifying the signals in undesired directions (interference) through channel matrix orthogonality [126] and achieve optimal performance in Multi-User (MU) MIMO environment [127]. CSI estimation can be carried out at the receiver. The process of the ZF algorithm detection can be explained by the following weight matrix [128]:

$$
\boldsymbol{W}_{\boldsymbol{Z} \boldsymbol{F}}=\left(\boldsymbol{H}^{H} \boldsymbol{H}\right)^{-1} \boldsymbol{H}^{H}
$$

With uniform power allocation over the UEs, the achievable sum rate is given by [129]:

$$
R_{Z F}=\sum_{k_{u}=1}^{k_{u}} \log _{2}\left(1+\frac{P}{K \sigma^{2}}\left|h_{k} W_{k}\right|^{2}\right)
$$

where $(\boldsymbol{H})^{H}$ denotes a function of Hermitian transpose of $\boldsymbol{H}, k_{u}$ is the number of users, $W$ is the weight vector, $\sigma^{2}$ is the statistical information of noise [128].

ZF precoding nullifies the interference, which increases the noise level as a consequence. However, the MMSE trades-off interference suppression and noise. MMSE maximise the post-processing SINR by minimising the mean square error. The MMSE weight is given as:

$$
\boldsymbol{W}_{\text {MMSE }}=\left(\boldsymbol{H}^{H} H+\sigma^{2} \mathbf{I}\right)^{-1} \boldsymbol{H}^{H}
$$

with achievable sum rate given by [129]:

$$
R_{M M S E}=\sum_{k=1}^{k} \log _{2} 1+\left(\frac{\left|h_{k} W_{k}\right|^{2}}{\sum\left|h_{k} W_{k}\right|^{2}+\frac{K \sigma^{2}}{P}}\right)
$$




\subsection{Mm-wave For Backhaul Links}

The enormous amount of mobile traffic in next generation mm-wave BSs should be carried to the mobile core network through high capacity backhaul links. The current conventional MW links do not have enough capacity to handle this huge traffic. On the other hand, fibre optics can provide efficient links with very high capacity to connect the high capacity small-cells. However, connecting fibre links to large numbers of small-cells is laborious and too expensive, due to the dense deployment trend in mobile networks [78][130].

Therefore, providing a flexible and efficient wireless backhaul (WiB) approach to replace the fibre links is a promising alternative [131]. WiB will lower the cost of wiring,digging/trenching to lay fibre in the ground and will minimise the labour and time required [77]. The mm-wave bands frequencies have typically been deployed for wireless backhaul with fixed LoS. Thus the networks backhaul of a $5 \mathrm{G}$ system will move from wire and optical fibre to mm-wave wireless backhaul, which will facilitate cheaper deployment connectivity among small-cell BSs. The provision of wireless backhaul will be divided in to different categories according to operating frequency band:

- Sub-6GHz band: This category adopts a tree architecture to connect the network nodes. A point-to-multi point (P2MP) link can ensure that the capacities of access links will not overload the network links, and single hop transmission can guarantee minimum latency. This scenario adopts LoS transmission only. In general, the link data throughput can support up to 200Mbps. Meanwhile, the point-to-point (P2P) solution adopts NLoS transmission and offers a lower link speed without the need for LoS [78].

LoS $6 \mathrm{GHz}$ - $42 \mathrm{GHz}$ Bands: This band has a significant role in backhaul for 3G and 4G. It is characterised by LoS microwave P2P links between $6 \mathrm{GHz}$ to $42 \mathrm{GHz}$ that operate on channels of up to $2 * 56 \mathrm{MHz}$. The antenna size is relatively large in order to achieve high gain and ensure efficient connectivity over long distances. However, 5G "massive" capacity requirements will require backhaul operating in higher frequency bands, beyond the $6-42 \mathrm{GHz}$ band [56], to utilise the wider bandwidth for higher data throughput links [132][133].

- $\quad$ E-band LOS (60- $70-80) \mathrm{GHz}$ : The E-band offers much higher capacity since it utilises much larger bandwidth. In small-cells deployment, this can be an advantage as there is low interference among E-band links. Moreover, the band is an unlicensed or lightly-licensed band, therefore, network operators will pay less or nothing to utilise this band [38][60][78]. Table 6 shows a summary of these backhaul options.

To summarise, the sub $6 \mathrm{GHz}$ band does not have sufficient bandwidth for backhaul links to support the high capacity $5 \mathrm{G}$ small-cells. Secondly, fibre links would not be a feasible solution for busy urban areas (e.g. street canyons) with dense small-cells deployment as they may require digging and trenching, which in some situations might be impossible. The 6$42 \mathrm{GHz}$ band has been used for $3 \mathrm{G}$ and $4 \mathrm{G}$ links, but is also not sufficient for $5 \mathrm{G}$. Therefore, a push toward the E-band is necessary, where a wide bandwidth (free or light licensed) is available. Table 7 shows some contributions to the field of using E-band as a backhaul link solution for $5 \mathrm{G}$ massive traffic growth. There are only a few studies on this subject.However, this subject needs careful study as the planning for backhaul link provision for future $5 \mathrm{G}$ cells is a challenging subject.

\subsection{Mm-wave Link Budget}

The factors responsible for specifying the BS link budget operating in the mm-wave are the transmit power, antennas gain, and path loss of the required path based on Tx-Rx separation [57][116]. The goal here is to provide a link capable of supporting multi-Gbps for users at maximum ISD of $200 \mathrm{~m}$ operating at the $28 \mathrm{GHz}$ band to benefit from wide bandwidth while simultaneously meeting hardware constraints, device implementation, and feasibility. Table 8 shows the link budget for different bands in addition to the $28 \mathrm{GHz}$ band. The $60 \mathrm{GHz}$ band has a valuable $7 \mathrm{GHz}$ of free licensed bandwidth. However, we might jeopardise the communication links for an outdoor scenario due to the high absorption by oxygen. Furthermore, the $(70,80) \mathrm{GHz}$ offers an additional $10 \mathrm{GHz}$ of bandwidth $(5 \mathrm{GHz}$ at $70 \mathrm{GHz}$ band $+5 \mathrm{GHz}$ at 80 $\mathrm{GHz}$ ), that can be deployed for mobile access as well as backhaul. In the E-band, a $2 \mathrm{GHz}$ bandwidth for mm-wave operation is a feasible option as shown in [81][84][134] for high data rate mobile access in future $5 \mathrm{G}$ systems. 
Table 6 Backhaul Link options for $5 \mathrm{G}$

\begin{tabular}{lllll}
\hline Backhaul & Capacity & Cost & Deployment & Reliability \\
\hline Fibre Link & Extremely high & High & Very difficult & Very high \\
sub 6GHz & Low & Low & Easy & High \\
$(6-42) \mathrm{GHz}$ & High & Low & Easy & Medium \\
E-Band & Very High & Low & Easy & Medium \\
\hline
\end{tabular}

Table 7 Some contributions on backhaul provision for $5 \mathrm{G}$ nodes

\begin{tabular}{llll}
\hline Ref & F(GHz) & Method & Description \\
\hline$[56]$ & E-band & Analysis & Small-cells backhaul candidates \\
{$[30]$} & All band & Analysis & Backhaul link budget analysis \\
{$[38]$} & 72 & Measurement & Indoor measurements and penetration loss for 73.5GHz \\
{$[60]$} & 73 & Measurement & Path loss and wave propagation for mobile access/backhaul \\
{$[56]$} & All & Measurement & Software defined 5G backhaul \\
{$[131]$} & $5,28,60$ & Simulation & High throughput and energy consumption backhaul link \\
{$[77]$} & 28 & Simulation & In-Band backhaul provision \\
{$[135]$} & 73 & Simulation & Combine access and backhaul on the same frequency. \\
{$[132]$} & 60 & Simulation & 60GHz antennas design for backhaul \\
\hline
\end{tabular}

\begin{tabular}{|c|c|c|c|c|}
\hline \multirow{2}{*}{ Link budget parameters } & \multicolumn{4}{|c|}{ Operating Frequency $(\mathrm{GHz})$} \\
\hline & 28 & 60 & 72 & 82 \\
\hline Tx Power(dBm) & 30 & 35 & 35 & 35 \\
\hline Tx gain $(\mathrm{dB})$ & 20 & 25 & 25 & 25 \\
\hline Rx gain $(\mathrm{dB})[57]$ & 15 & 15 & 15 & 15 \\
\hline Losses $(\mathrm{dB})$ & 20 & 20 & 20 & 20 \\
\hline $\operatorname{EIRP}(\mathrm{dBm})$ & 30 & 40 & 40 & 40 \\
\hline Distance (m) & 200 & 200 & 200 & 200 \\
\hline Path loss (dB) & 107.4 & 114.03 & 115.6 & 116.7 \\
\hline Rx power(dBm) & -62.41 & -59.03 & -60.61 & -61.74 \\
\hline $\mathrm{BW}(\mathrm{GHz})$ & 1 & 2 & 2 & 2 \\
\hline Noise Figure (dB) & 10 & 10 & 10 & 10 \\
\hline Noise $(\mathrm{dBm})$ & -74 & -71 & -71 & -71 \\
\hline Noise PSD (dBm/Hz) & -174 & -174 & -174 & -174 \\
\hline $\operatorname{SNR}(\mathrm{dB})$ & 11.58 & 11.97 & 10.39 & 9.26 \\
\hline
\end{tabular}

\section{Relevant and open issues in $\mathbf{m m}$-wave 5G}

Due to their different characteristics, mm-wave networks will introduce major challenges that require specific design considerations for all layers. Some research contributions which consider mm-wave designs are summarised in Table 9, where the mm-wave link budget is analysed in [4] for different bands, and the spectrum implication of mmwave are discussed in [23]. Furthermore, a conceptual cost-capacity study to evaluate 5G profitability is discussed in [136] to maintain excessive user demands, and in [137], where the MAC layer design to suit mm-wave waveform is investigated. The work in [138] defines and evaluates important metrics for multimedia QoS and considers these factors within the mm-wave 5G framework, while in [139], a D2D framework is presented, where the authors have proposed a resource sharing scheme with non-interfering D2D links. The frequent handoff in ultra-dense networks in mm-wave is investigated in [140], where the authors have estimated the average handoff intervals in many scenarios in dense networks with redundant access points. The authors in [141] have proposed a $5 \mathrm{G}$ framework for ultra-low latency with $0.1 \mathrm{~ms}$ frame duration in the mm-wave frequency band, while in [142], the authors have proposed a compression and relaying algorithm for outdoor wireless video applications. Transmission of HD video is a promising option for mmwave, since a very high data rate is possible. The design and implementation of mm-wave mobile device transceivers and array antennas that suit mm-wave propagation characteristics are investigated in [57][143][144][145][146]. 
Table 9 Other contributions on mm-wave

\begin{tabular}{llll}
\hline Ref. & F $(\mathrm{GHz})$ & Method & Description \\
\hline$[4]$ & $(28,72)$ & Analysis & Link Budget Analysis \\
{$[23]$} & All & Analysis & Spectrum management implications \\
{$[136]$} & All & Analysis & Cost-Capacity Analysis \\
{$[137]$} & 60 & Analysis \& Sim. & MAC-layer Perspective \\
{$[138]$} & $(60,70)$ & Simulation & QoS multimedia scheduling \\
{$[139]$} & 60 & Simulation & A framework for D2D \\
{$[140]$} & All & Simulation & Handoff characteristics \\
{$[141]$} & All & Simulation & Low latency radio interface \\
{$[147]$} & 28 & Simulation & Antenna clustering Schemes \\
{$[142]$} & All & Analysis \& Sim. & Wireless Video Systems \\
{$[143]$} & 28 & Simulation & Large scale antenna for mobile device \\
{$[144]$} & 28 & Fabrication & Low cost PCB mm-wave path antenna \\
{$[57]$} & 28 & Analysis & Antenna array design for BS and UE \\
{$[145]$} & 28 & Simulation & Phased array antenna for UE handset \\
{$[146]$} & $(60,70)$ & Simulation & Hybrid transceiver design \\
\hline
\end{tabular}

The following sections relate to various key considerations when adopting mm-wave for $5 \mathrm{G}$ :

\subsection{Waveform Considerations}

The waveforms to be used in 5G radio access should be capable of managing a set of 5G requirements, such as high spectral efficiency, low latency and limited cost and complexity [148]. Some contributions have considered OFDM as the $5 \mathrm{G}$ waveform. This is mainly due to its ability to manage multipath channels in a cost effective manner, flexibility in supporting multiple bandwidths, simpler equalisation, and its straightforward extension to MIMO [4]. However, OFDM is not without drawbacks, and its adoption in the next generation networks cannot be taken for granted. In this context, the major drawback of OFDM is the high Peak to Average Power Ratio (PAPR), which degrades the efficiency of PA. On the other hand, new waveforms such as Filter Bank Multi Carrier (FBMC), Sparse Coded Multiple Access (SCMA), and Non-Orthogonal Multiple Access (NOMA) have the ability to improve 5G spectral efficiency. For example, NOMA can improve the data throughput in macro-cells by up to $30 \%$ over orthogonal multiple access schemes when used with a receiver implementing Successive Interference Cancelling (SIC), even for high mobility users. Higher gains are expected when advanced power control is utilised [73]. FBMC generalises the multi carrier concept. The key idea is that the modulated signals on the subcarriers are filtered by an optimised prototype filter (in order to suppress signals' side lobes, eventually making them strictly band-limited). OFDM spectral efficiency is limited by the Cyclic Prefix (CP) and by its large side lobes (that need null guards, which eventually decrease spectral efficiency). FBMC is an alternative approach to OFDM since it has a higher spectral efficiency. FBMC uses common Frequency Division Multiple Access (FDMA) without subcarrier overlap, while the lack of side lobes allows increased spectral efficiency [148].

Another possible alternative to OFDM transmission is the Single Carrier Null Cyclic Prefix (NCP-SC), which has been proposed for mm-wave frequencies. In this system, the normal cyclic prefix is replaced with a group of zeros and added after the block of data, which acts as the cycle prefix for the next data block [149][150]. SC has lower PAPR compared to OFDM and therefore has been used by the IEE802.11ad standard for reliable transmission [151]. IEE802.11ad and IEEE 802.15.3c also use OFDM to combat ISI in frequency-selective fading in the $60 \mathrm{GHz}$ band to achieve multi Gbps data rates over short distances [152].

One of the inevitable impairments of operating at mm-wave is increased phase noise [149]. The Phase noise of an oscillator increases as the frequency increases. The phase noise generates a Common Phase Error (CPE) in OFDM and SC systems, which create ICI in OFDM systems and inter-symbol interference (ISI) in SC systems [153]. Multi carrier systems such as OFDM are more sensitive to phase noise than SC systems, because it damages the subcarriers orthogonality [154]. The phase noise can be high enough to significantly compromise performance in mm-wave systems [153].

In order to adopt OFDM in mm-wave communications, mitigation of the phase noise in OFDM-MIMO systems in mmwave was proposed in [155]. The authors proposed a model for OFDM precoding with Pilot-assisted Estimation and 
Compensation (PEC). This model helps estimate the channel response as well as the CPE caused by phase noise, which significantly improves the system performance.

Furthermore, DAC/ADC sampling processing needs to process wide band signals in mm-wave, which results in considerable power consumption. In [151], the authors have proposed a power-efficient subsampling system to improve OFDM performance for mm-wave communications. The proposed OFDM with a low resolution ADC architecture has shown a power consumption reduction in the $60 \mathrm{GHz}$ band. Furthermore, an efficient algorithm has been developed by [152] based on low resolution ADC for optimal data detection in mm-wave OFDM systems. These results assess the feasibility of CSI acquisition using low resolution ADC without significant pilot overheads. The authors in [156] have proposed a Constant-Envelope OFDM (CE-OFDM) for mm-wave communication. The CE-OFDM exploits the frequency diversity effectively, which increases the coverage probability of mm-wave in multipath channels and supports a very high data rate scenario.

For mm-wave band, the OFDM system is more sensitive than the SC system is to the PAPR [150].High PAPR due to IFFT/FFT operation makes OFDM vulnerable to the PA nonlinear and phase noise [153]. In this regard, the CE-OFDM can significantly reduce the PAPR while maintaining most the advantages of OFDM [113]. In addition, Discrete Fourier Transforms OFDM (DFTS-OFDM) can lower the PAPR on account of the more complicated system design [157]. The authors in [158] suggest that the SC mode in IEEE 802.11ad can cause performance degradation in a multipath channel.They proposed DFTS-OFDM as an alternative for this standard in order to improve its peak data throughput performance without changing the packet structure. In addition, a waveform with high PAPR requires significant power back-off at the PA to avoid non-linear signal distortion, which reduces PA efficiency [158]. Further contributions were investigated to reduce PAPR in OFDM. For instance, Unique Word UW DFTS-OFDM reduces the PAPR compared to normal OFDM [158], and trellis-coding is proposed in CE-OFDM systems in order to reduce PAPR [156]. These contributions suggest that these novel OFDM schemes are strong candidate for the $5 \mathrm{G}$ waveform.

\subsection{Advanced channel coding}

State-of-the-art channel coding schemes can provide data rates closer to channel capacity fundamental limits. Advanced channel coding techniques with MIMO schemes are now deemed to be anindispensable aspect of enhanced 4G and future $5 \mathrm{G}$ and beyond systems. One of the state-of-the-art research areas leading to PHY-layer enhancements are advanced turbo-like coded MIMO schemes, which can be used to compensate for channel impairments of mm-wave systems [159]. Turbo-like coded MIMO schemes are advanced versions of classic turbo coded systems used in 4G network. High performance turbo-like codes have been designed in [160] using algebraic derivations based permutations. This scheme supports twin/triplet antenna bit-level space-time codes with binary turbo-like codes. Using this model, the BER performance of space-time turbo coded MIMO communication systems in waterfall and flare regions shows significant improvement compared with row-column block interleaved systems. Additionally, Faster Than Nyquist (FTN) transmitters are proposed in [161], where subframes are encoded using multiple turbo-like codes. This model has the potential to improve the BER performance and reduce decoding latency.

Furthermore, rateless coding schemes have been proposed in order to improve coding efficiency. In rateless coding, the transmission is initiated with high-speed transmission, following which, additional parity data is transmitted until the receiver decodes successfully. Therefore, the temporal channel condition transmission efficiency is significantly improved, without requiring channel feedback from the receiver [162]. Unlike typical Adaptive Modulation and Coding (AMC) schemes, rateless coding schemes are robust to erasures. Rateless coding is widely used in cooperative relay networks as a low-complexity transmission scheme to improve system reliability and security [163]. In addition, a new rateless coding based on the extendibility of Polar Codes is proposed in [164].The new proposal can be applied for time in-varying channels without the need for CSI feedback, and hence support more efficient and reliable links.

\subsection{Providing MIMO link in mm-wave LoS}

Supporting higher data throughput without increasing the system bandwidth simultaneously motivates the emergence of MIMO communication systems. MIMO can support multiple parallel streams by using closely separated antenna elements at both the transmitter and receiver. 
In mm-wave a MIMO system should create multiple parallel streams. However, in the LoS [61], mm-waves have higher dependency on LoS transmission. This system is called mm-wave MIMO. The fundamental theory of this network scheme was first introduced in [165] as shown in Fig.19. In such configurations, both transmitter and receiver are equipped with an antenna array of dimension $1 \times n_{j}$ or $n_{i} \times n_{j}$ elements with higher separation among elements. The default spacing among antennas elements in the conventional mobile network is half a wavelength.Higher spacing can improve the MIMO channel by decreasing the correlation coefficient between LoS streams, and eventually improve the average data throughput of the system. However, when the wavelength is too long; as in the case sub $3 \mathrm{GHz}$, higher spacing would create an unrealistic array size. In mm-wave, this is not a problem because of the very short wavelength of this band. Therefore, through optimised antenna spacing, spatial streams can be generated to support Spatial Multiplexing (SM) operation in LoS, which will be the environment of mm-wave communications.

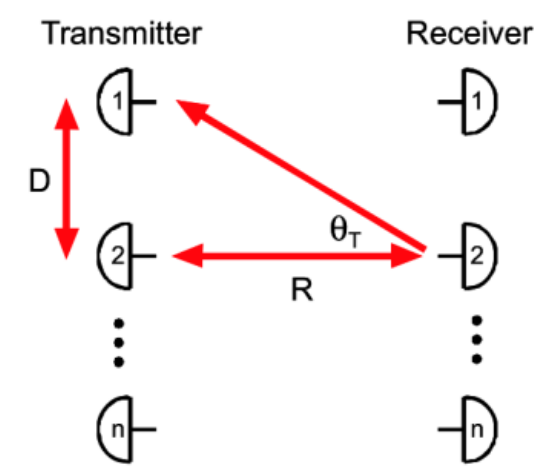

Fig.19. LoS MIMO system in the mm-wave band [61].

The Correlation Coefficient (CC) is the relationship between the radiation patterns of the antennas in an array, which indicate how independent the antennas are. The correlation is used as a performance metric in MIMO to measure the efficiency of the antennas. Therefore, using the correlation coefficient, a network designer is able to manage the coupling between the antennas in the array. To reduce the mutual coupling, the correlation coefficient between two antennas needs to be reduced. There are two approaches to calculating the correlation coefficient between antennas, namely: the antennas radiation patterns and the S-parameters approach [166].

The Envelop Correlation Coefficient (ECC) using the radiation pattern approach of two antennas is represented by:

$$
\rho=\frac{\left|\iint_{4 \pi}\left[\overrightarrow{\boldsymbol{F}}_{\mathbf{1}}(\theta, \emptyset) \cdot \overrightarrow{\boldsymbol{F}}_{\mathbf{2}}(\theta, \emptyset)\right] d \Omega\right|^{2}}{\iint_{4 \pi}\left|\overrightarrow{\boldsymbol{F}}_{\mathbf{1}}(\theta, \emptyset)\right|^{2} d \Omega \iint_{4 \pi}\left|\overrightarrow{\boldsymbol{F}}_{\mathbf{1}}(\theta, \emptyset)\right|^{2} d \Omega}
$$

where $\rho$ is the ECC:

$$
0 \leq \rho \leq 1
$$

$\boldsymbol{F}_{\mathbf{1}}$ and $\boldsymbol{F}_{\mathbf{2}}$ are the radiation patterns of the two antennas (vector function in spherical coordinates).

$\theta, \phi$ represents the spherical angles (elevation, azimuth). If the two antennas are identical (so that $\boldsymbol{F}_{\boldsymbol{l}}=\boldsymbol{F}_{2}$ ), the correlation coefficient would be 1 . If they are completely independent, the correlation would be 0 . However, this approach is very complex and time consuming.

On the other hand, a second approach can be modelled using the S-parameter calculation. This approach is quicker and simpler than the approach in eq. (16) because the S-parameter calculation does not use spherical radiation patterns. The S-parameter correlation coefficient is represented by:

$$
\rho=\frac{\left|S_{11}^{*} S_{12}+S_{21}^{*} S_{22}\right|}{\left.\left(1-\left(\left|S_{11}\right|^{2}+\left|S_{21}\right|^{2}\right)\right)\left(1-\left|S_{22}\right|^{2}+\left|S_{12}\right|^{2}\right)\right)}
$$

The correlation between two elements in the antennas array depends on the spacing between elements in terms of wavelength $\left(D_{\lambda}\right)$, mean angle of arrival (AoA) of the incoming signals, and the Power Azimuth Spectrum (PAS). Because the diversity gain decreases when the correlation between the antennas increases, the antenna spacing has to be large enough to reduce the correlation [128]. In [62], The authors have used a 4x4 MIMO system, where the BS antenna array configuration used is a uniform linear array (ULA). In ULA, all antenna elements are vertically polarised with a zero 
slant angle. This is known as a COPOL array, as illustrated in Fig.20a. The configuration can be amended to simulate a cross polarised (XPOL) antennas array, by setting the antennas elements slant angle to $-45 / 45$, (known as X polarisation) as shown in Fig.20b. The UE array configuration is illustrated in Fig.20c.

In system modelling, the ULA array has been selected for both BS and UE, a zero slant angle has been set for all elements in order to represent a COPOL. 21 cells have been used in the modelling with ten users allocated per cell (total is 210 UEs). The increase in antenna spacing $\left(d_{h}\right)$ in term of wavelength $(\lambda)$ will enable the system to avoid undesired antenna effects such as high correlation, high mutual coupling, changing beam patterns, and high mismatch loss [167]. The lack of angular spread in LoS transmission can be compensated by increasing antenna spacing [168]. Higher spacing among elements will minimise the correlation among their channels in order to transmit simultaneous and independent data. streams[169].

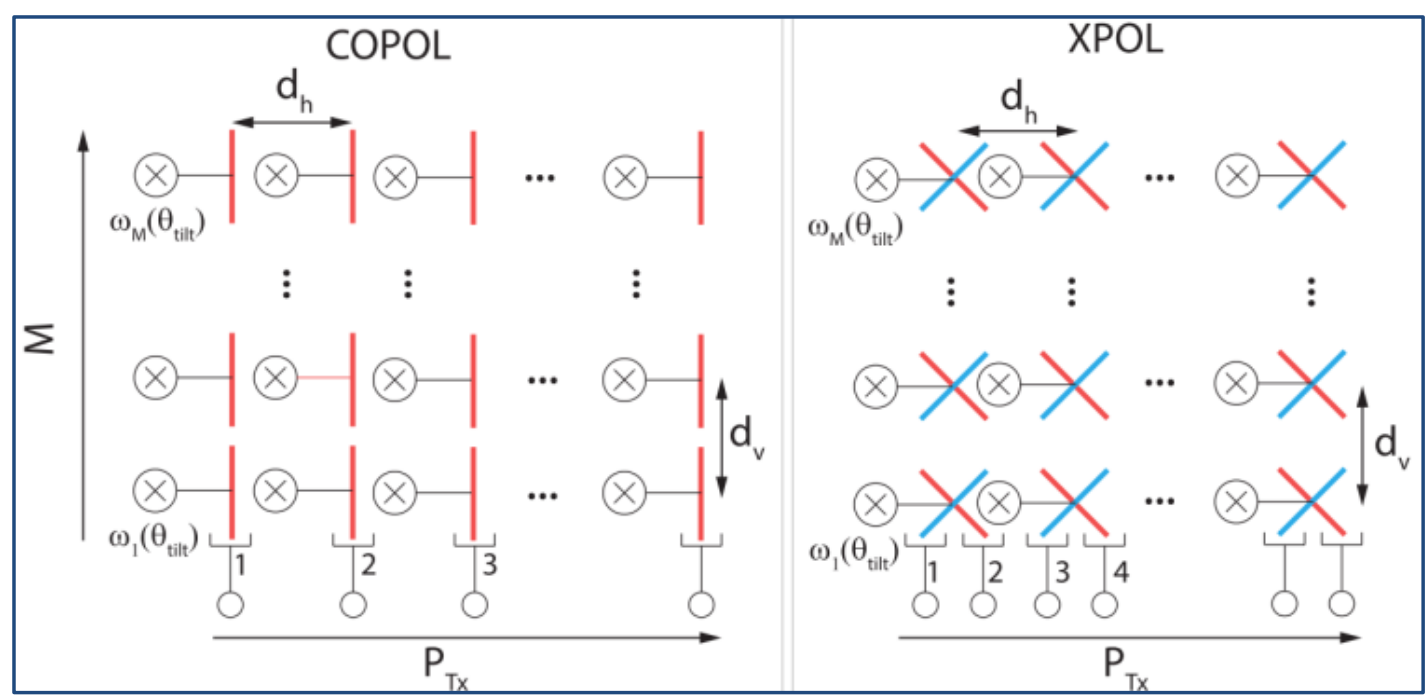

(a)

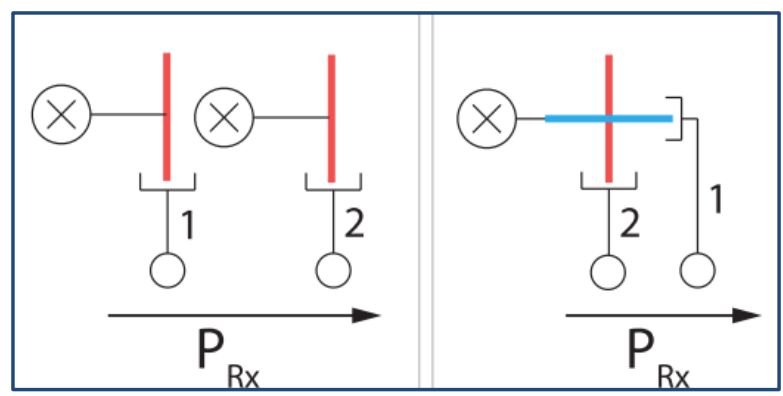

(c) (b)

Fig.20. ULA configuration at (a,b) for BS array and (c) for UE array.

The results have shown a remarkable gain in average cell throughput as well as per user cell-edge data throughput compared to a conventional array of $0.5 \lambda$ spacing. Furthermore, with shorter wavelengths in mm-wave bands, higher element spacing will yield a realistic array size.Therefore higher spacing of 10 to $40 \lambda$ has been considered [62].

In this configuration, UEs at the cell-edge have experienced higher SINR, which consequently increased the data throughput. The results demonstrated in Fig.21 show the improvement in the average cell data throughput as well as the per user cell-edge data throughput. In Fig.22, a Cumulative Distribution Function (CDF) is presented for all 210 UEs, which shows the data throughput averaged over the 210 UEs, with multiple array spacing. Significant improvement in data throughput is demonstrated when higher spacing is used [62]. 


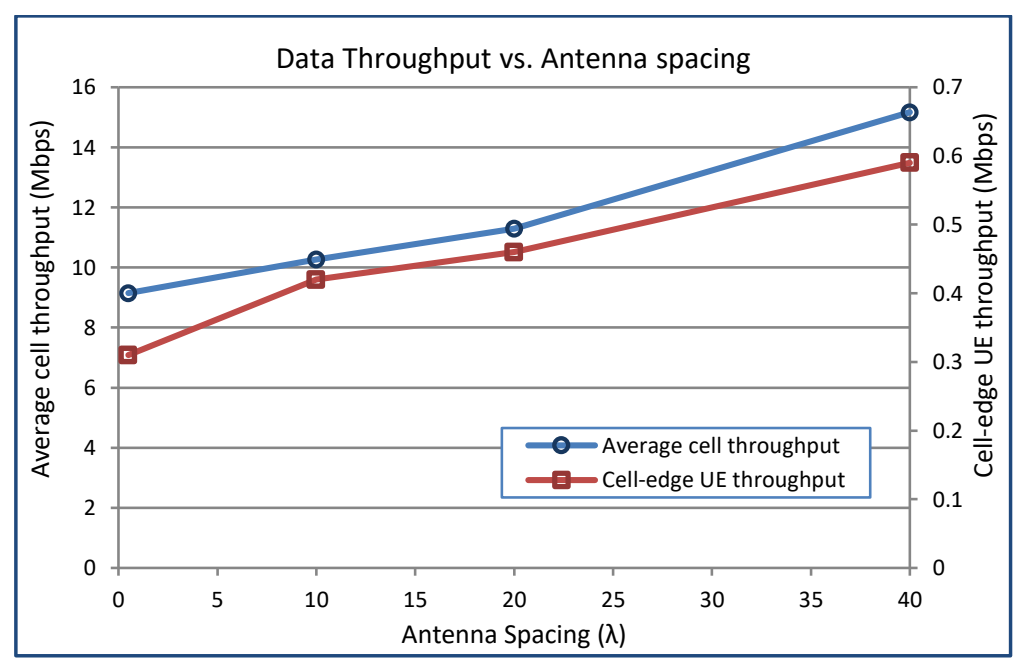

Fig.21. Data throughput vs. array spacing [62].

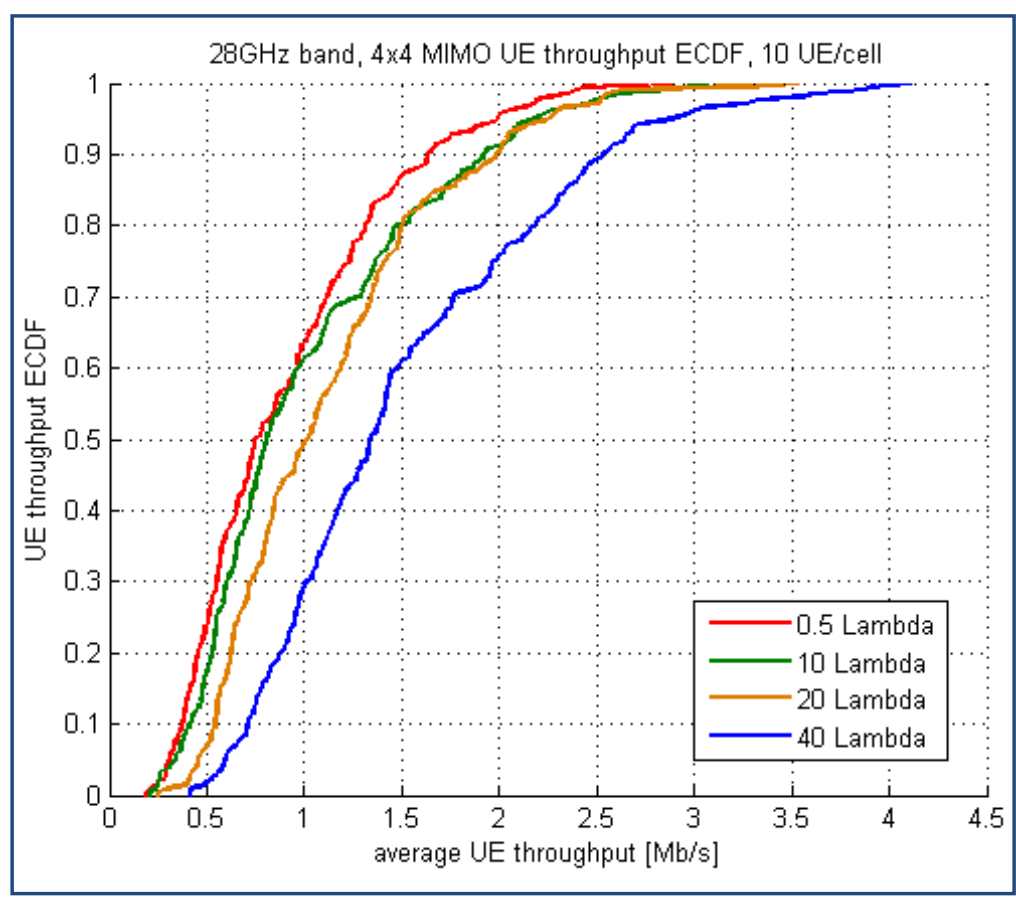

Fig.22. CDF of average UE data throughput vs array spacing [62].

\subsection{Interference Coordination in mm-wave UDN}

Inter Cell Interference (ICI) is one of the challenging issues that degrades mobile network performance. ICI arises in next generation networks, as the trend is to deploy denser networks. ICI degrades data throughput, particularly at celledge areas, which minimises network spectrum efficiency. Cellular networks have two techniques for frequency assignment. In the first technique, the BSs transmit at different carrier frequencies. Here, each sector uses only $25 \%$ of the total bandwidth and the Frequency Reuse Factor (FRF) is $25 \%$. This approach is called $\mathrm{FRF}_{3}$ which significantly reduces the ICI among the surrounding cells. However, it also reduces the spectral efficiency. The scheme is adopted in 2G GSM systems. In 3G/4G networks, BSs utilise the whole bandwidth (with FRF of 100\%) The scheme is called FRF . FRF $_{1}$ increases the spectral efficiency at the expense of increased ICI, particularly for cell-edge users [102].

An alternative approach can trade-off both techniques [170]. Here, each BS transmits at the same carrier frequency in the centre zone, utilising the majority of the bandwidth for the users in this area, while the outer zone users use the rest of the bandwidth. In order to reduce the ICI, this scheme is called Fractional Frequency Reuse (FFR) [102][171]. FFR splits the BS coverage area into inner and outer zones according to the threshold SINR $\left(\gamma_{t h}\right)$ of the users, as shown in Fig.23. 
Different types of FRFs are implemented in these zones in order to minimise ICI as well as to increase the spectral efficiency. Details of the FFR algorithm and parameter settings are shown in [170].

Therefore, with homogenous power allocation, a UE at the $(x, y)$ point within the BS coverage area will be located either in the inner or cell-edge area as follows:

$$
U E_{i} \in\left\{\begin{array}{l}
\gamma_{i, U E_{(x, y)}} \leq \gamma_{t h} U E_{(x, y)} \in F R F_{3} \\
\gamma_{i, U E_{(x, y)}}>\gamma_{t h} U E_{(x, y)} \in F R F_{1}
\end{array}\right.
$$

The FFR scheduler splits the total bandwidth $\mathrm{B}_{\mathrm{T}}$ between these inner and outer zones. Therefore, in a default three sector $\mathrm{BS}$, the total bandwidth is given by:

$$
B_{T}=B_{\text {inn }}+B_{\text {out }}
$$

where $B_{i n n}$ is the bandwidth allocated to the inner zone and $B_{\text {out }}$ is the bandwidth allocated to the outer zone.

For more efficient bandwidth partitioning, $\beta_{F R}$ the normalized FR bandwidth is defined as:

$$
\beta_{F R}=\frac{B_{\text {inn }}}{B_{T}}, \text { where } 0 \leq \beta_{F R} \leq 1
$$

Hence, the total available bandwidth of a single sector is:

$$
B_{F F R}=\beta_{F R} \cdot B_{T}+\frac{1}{3}\left(1-\beta_{F R}\right) B_{T}
$$

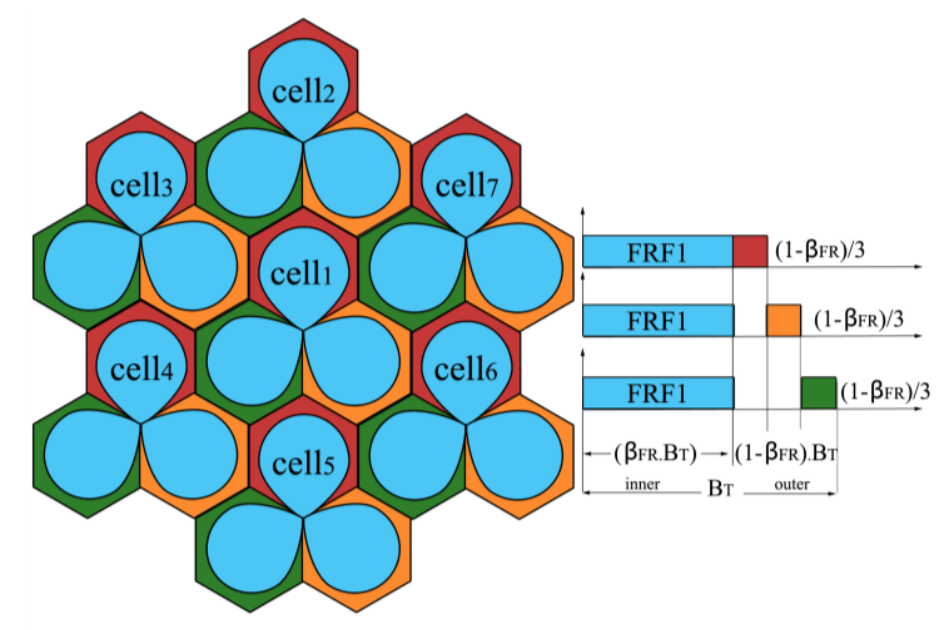

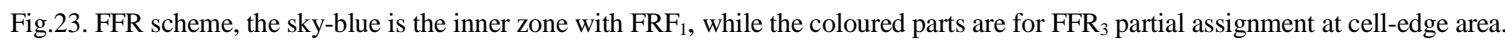

Fig. 24 shows the SINR to throughput mapping in a FFR cellular network. Here, $\beta_{F R}=0.7$ and $\gamma_{t h}=0 \mathrm{~dB}$. In this scheme, the BS coverage area is divided into centre and cell-edge areas, with $\mathrm{FFR}_{1}$ and $\mathrm{FFR}_{3}$, respectively. The data throughput of the cell-edge area has improved to $9.26 \mathrm{Mbps}$, with a gain of $47.5 \%$, and the cell data throughput has improved to 226.7 , with a gain of $20 \%$. The peak throughput is also improved to $66.3 \mathrm{Mbps}$, with a gain of $16 \%$ over the FFR $\mathrm{F}_{1}$ network. Lower values of $\beta_{F R}$ can improve the data throughput of the cell-edge area, but will degrade the average and peak data throughput. Fig.25 shows the gain in peak, average and cell-edge data throughput with FFR ICI coordination as a function of $\beta_{F R}$. 


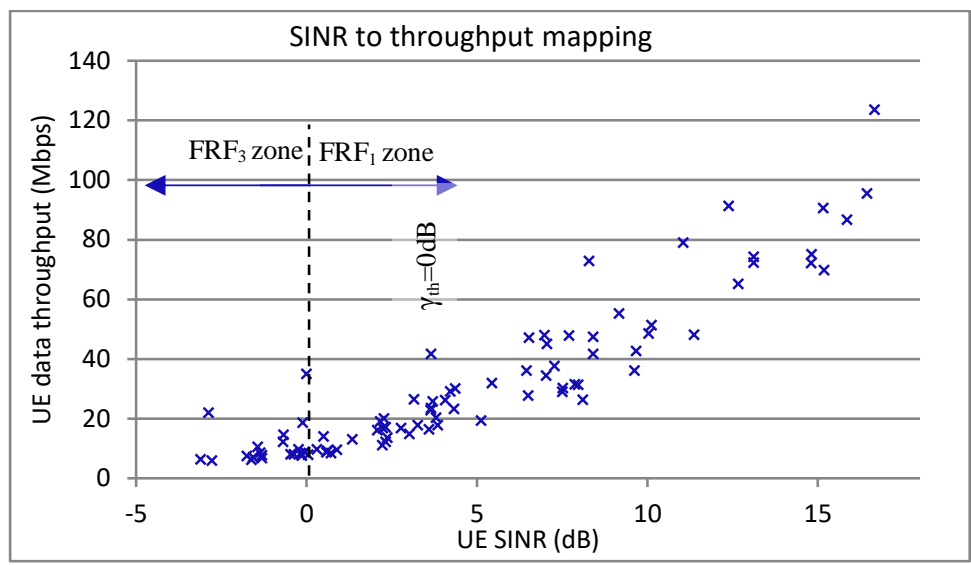

Fig.24. SINR to TP mapping of FFR network, with $\gamma_{\mathrm{th}}=0 \mathrm{~dB}$ and $\beta_{F R}=0.7$ [170].

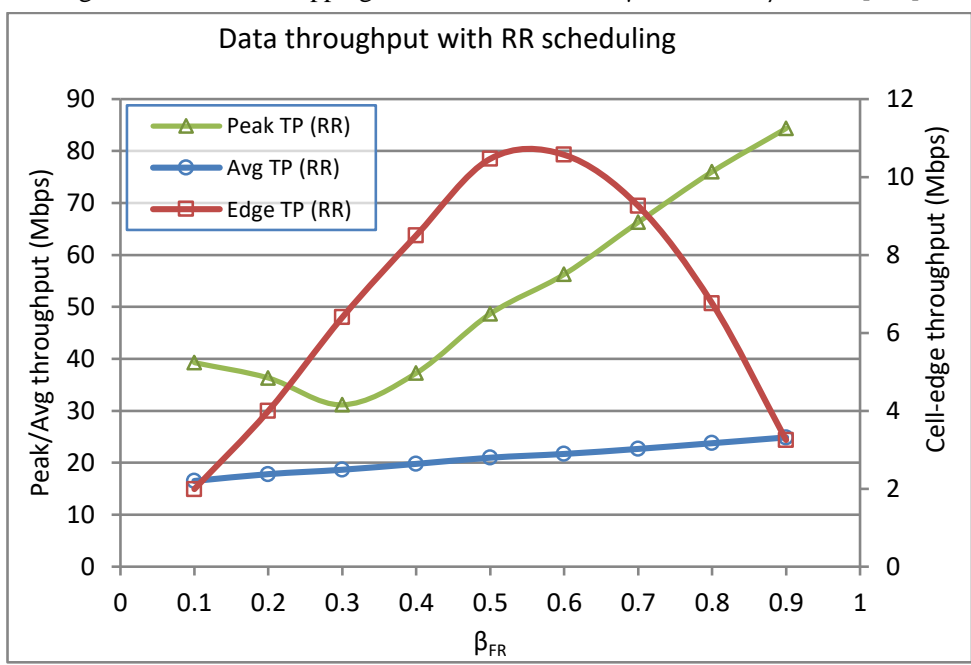

Fig.25. The gain in data throughput in FFR network [170].

\subsection{Soft Densification in 5G}

The purpose behind the emergence of mm-wave communications and network densification is to enable higher increases in the capacity of $5 \mathrm{G}$ networks with efficient utilisation of all their resources. Based on Shannon theory, channel capacity can be expressed by [172]:

$$
C=\sum_{\text {HetNet Channel }} \sum_{i} B_{i} \log _{2}\left(1+\gamma_{i}\right)
$$

where $B_{i}$ is the bandwidth allocated for the channel $(i)$ and $\gamma_{i}$ is the SINR. From this equation, the system capacity $C$ is equivalent to the sum rate of all HetNets cells and all channels. Therefore, the network capacity can be increased by: increasing the system coverage via HetNets such as small-cells, relays, and RRHs; by increasing the total channel by using CLSM, multi-point transmission and massive MIMO;by increasing the system bandwidth via mm-wave spectrum and cognitive radio techniques; and by improving the SINR via, beamforming and large scale antennas.

Network densification is the deployment theme of next generation 5G networks. Generally densification can be addresssed by frequency, by using higher carrier frequency with wider bandwidth, and via space, by using large number of antennas, sectors, and denser cell installations [89][87]. Excess interference due to a UDN network has to be coordinated in order to achieve the best gain for this dense scheme. Massive MIMO with beamforming offers significant interference reduction by minimising the interference from unwanted cells (interferers). However, massive MIMO needs significant improvement to avoid its drawbacks such as pilot contamination, unrealistic physical architecture, and inaccurate CSI estimation. On the other hand, fractional frequency reuse (FFR) has the potential to improve the UDN network. In the FFR scheme, every BS transmits using the same carrier frequency in the inner zone using most of the allocated bandwidth, while the cell-edge area uses the rest of the bandwidth in order to reduce interference. The FFR scheme divides the BS coverage area into two zones - an inner zone and an outer zone. Different frequency reuse factors 
(FRFs) schemes are implemented in these zones in order to reduce ICI among nearby cells [170].

To avoid the need to install new sites which would require substantial amounts of labour and time, an alternative option is Soft Densification. In soft densification, distributed RRHs with fibre optic links are used in order to densify the network in high traffic demand areas. This paradigm is a cost and time effective solution compared to normal densification. RRHs offer a UDN network with less equipment and less interference. As demonstrated in Fig.26, soft densification has the potential to achieve a significant gain over normal densification. In this scenario, RRHs have been distributed apart from the central node according to:

$$
d_{R R H}=\frac{1}{3} I S D
$$

where $d_{R R H}$ represents the distance of RRH from their central node. Therefore, with 300m ISD, when using RRHs the effective ISD will be $100 \mathrm{~m}$, with a further gain in average data throughput.

Densification over frequency on the other hand has higher gains due to the direct proportional relationship as shown in Shannon's capacity theorem in eq.6. When higher improvement in capacity is required, additional spectrum is needed. All mobile generations have improved their QoS by utilising additional bandwidth. Therefore, 5G will likely utilise higher bandwidth in the mm-wave band [5]. In this work, up to $500 \mathrm{MHz}$ bandwidth, which is located at the $26 \mathrm{GHz}$ pioneer band, has been used to show the improvement of increasing the system bandwidth of a 5G network, using a CBS and DBS network architecture. Significant gains are shown in Fig.27 as the system bandwidth is increased The most improvement is achieved using a DBS network architecture, where three RRHs have been used per each cell. Foe an average cell performance at $500 \mathrm{MHz}$ bandwidth, there is around $30 \%$ gain in DBS architecture over the CBS network architecture.

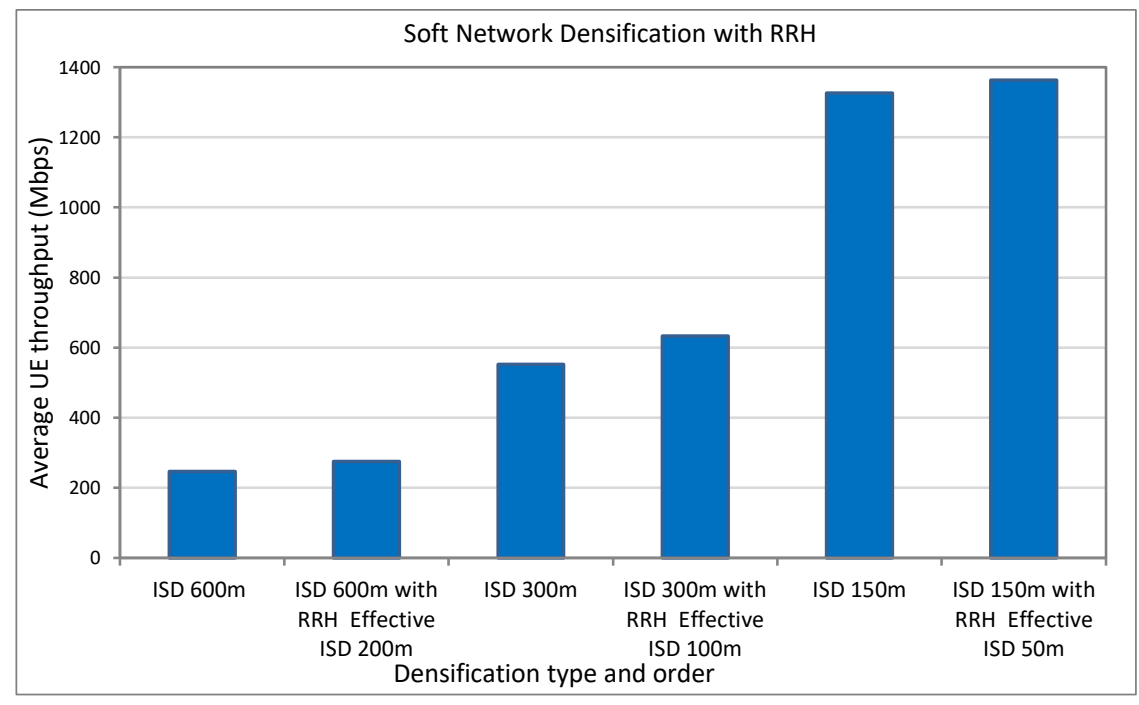

Fig.26. Soft densification gain over normal densification. 


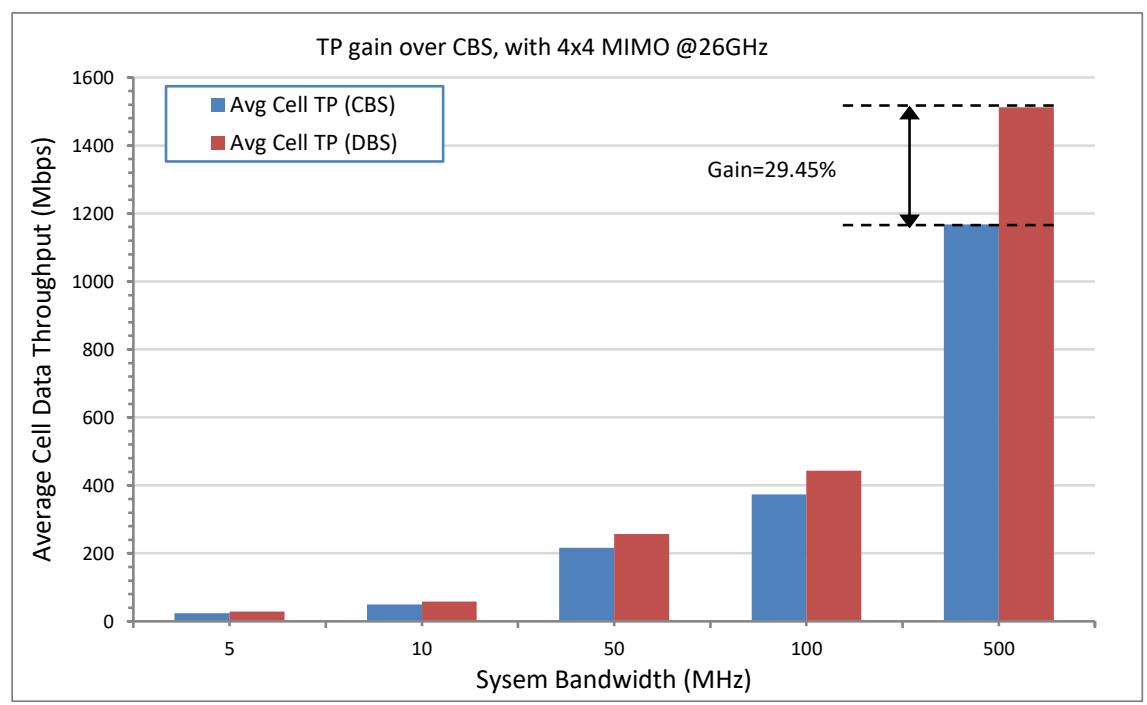

Fig.27. Data throughput vs. system bandwidth.

\subsection{Handoff Issue}

Dense deployment of mm-wave small-cell nodes is essential to offload congested macro-cells and to enhance the signal power (by reducing path loss). In the event of shadowing or LoS blockage, users will handoff (HO) to a nearby node in the cluster. These HOs could be frequent as users move within the cluster [140].

In order to minimise $\mathrm{HO}$ rates in a HetNets $5 \mathrm{G}$ network, a Control/User plane (C/U plane) split architecture can be adopted in this context [80]. The $\mathrm{C} / \mathrm{U}$ split scheme is used to enable UEs to receive system control signalling and initiate network access requests to a BS, while a different BS assigns resources with high data rates transmission. Therefore, system control signalling and user data services can be provided by two different/specialised BSs or can be implemented separately and independently in a single BS. In HetNets, the approach is to have the macro BS providing the signalling service for the whole area, while small-cell BSs assign data resources for high data rate transmission with a low control overhead using an appropriate air interface [130]. A similar approach can be considered in mm-wave 5G HetNets with a $(\mathrm{C} / \mathrm{U})$ plane split.A potential architecture is to have macrocells working as anchor cells and operating in the licensed low frequency band (sub 3GHz), while small-cells (phantom-cells) work as booster cells that provide high speed data using licensed and unlicensed spectrum in low frequency and mm-wave bands, deployed both indoors and at outdoor hotspots [73]. In that way, moving from one small cell into another will not necessitate a handover, as the control signal is still from the same macro cell.

Dual connectivity is an important topic for reducing HO rates in a mm-wave UDN network [173], where dual connectivity protocols enable UEs to maintain PHY connectivity to 4G and 5G, simultaneously. In this work [173], the authors show that when UEs are in shadow with respect to all surrounding mm-wave nodes, a dual connectivity proposed framework can address that, with the aid of a LTE macro node. This framework collects measurements and performs HO to fall back to the marco LTE service, which significantly improves the HO latency, system signalling, and average packet loss.

An alternative solution to minimise HO is to use a DBS architecture. To demonstrate its impact, we have conducted a system level simulation in order to measure the impact of distributed RRHs in minimising the excess handoff in a UDN environment. In the simulation, a Walking Model (WM) has been setup for all UEs, which makes the UEs walk in a specific route, such as linear, random, and starburst [174]. A WM is needed as it simulates UEs moving together from one point to another in the ROI. As an example for WM, where a UE starts walking from a cell, the destination cell of that UE after random movements is determined in [175]. While in [176], it introduces probabilistic walking models on the number of steps a UE should walk in order to leave ROI. The handoff decision of the target BS is made based on the best SINR value of the surrounding cells. For fair comparison, the same model has been used for UEs movement under both CBS and DBS networks. 
The impact of using a DBS architecture on handoff is shown in Fig.28. Low handoffs in this architecture are when a UE moves according to the walking model and passes into the RRH of a stronger SINR, no handover will be initiated. While in the case of CBS, every time a UE moves into a stronger SINR cell, a handover occurs. The excess handovers create more signalling overhead, which decreases the performance metrics and the data throughput. Therefore, a DBS architecture has superior performance over CBS in a UDN scheme, due to the potential handover increase in this dense environment.

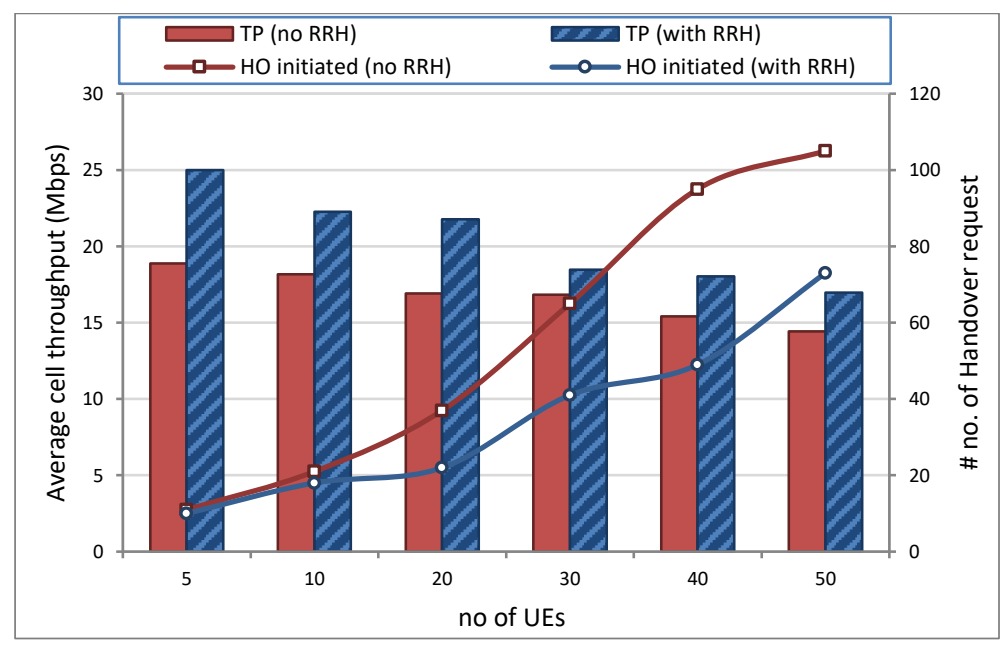

Fig.28. Decreasing excess HO with DBS.

\subsection{Supporting massive M2M traffic}

One of the main challenges of $5 \mathrm{G}$ networks is how to support massive numbers of wireless devices in the Internet of Things (IoT). The IoT will interconnect billions of devices under M2M. The emergence of low power consumption wireless technologies is one of the main enablers of the IoT, and $5 \mathrm{G}$ will be the key in establishing IoT as a tangible concept to tusers[177]. According tor Cisco, a key enabling factor contributing to IoT traffic growth is the proliferation of wearable devices and sensors, such as smart watches, smart glasses, smart health sensors, fitness sensors, navigation and tracking devices, and so forth [1]. The major traffic growth is anticipated to occur in M2M communications. M2M links will reach more than $26 \%$ of total connections by 2020, as shown in Fig.29.

In [178], M2M communication has been studied at a higher carrier frequency of $40 \mathrm{GHz}$ with a $5 \mathrm{~m}$ link distance. tThe study has been conducted for the healthcare sector to assess the feasibility of using mm-wave links in M2M. An experimental channel measurement has been conducted by [179] at $60 \mathrm{GHz}$ for the indoor scenario, which shows that channel interdependency analysis can help predict channel parameters in a M2M sensor network. In [180], a 60 GHz channel capacity and bit rate analysis has been presented, and compared with the sub $6 \mathrm{GHz}$ links from IoT perspectives. The authors have found that the use of the mm-wave band is a viable solution for the provision of high performance links to support IoT. The authors in [177] investigate the joint use of multiple antennas, beamforming, and the adoption of $\mathrm{mm}$-wave wave at the $28 \mathrm{GHz}$ band. This is to cope with the massive traffic generated by the M2M connections, to support fixed sensors and wearable sensors in 5G in the IoT environment. While in [19], the use of a DBS architecture for massive Machine Type Communications (mMTC) is demonstrated in terms of spectral efficiency and fairness, where a DBS architecture outperforms a CBS network in all performance metrics. Further studies and investigations in this area are needed in order to assess the feasibility of using a higher carrier frequency at mm-wave for M2M links, and to provide optimised algorithms for efficient resourses allocation to users and machines in the IoT environment. 


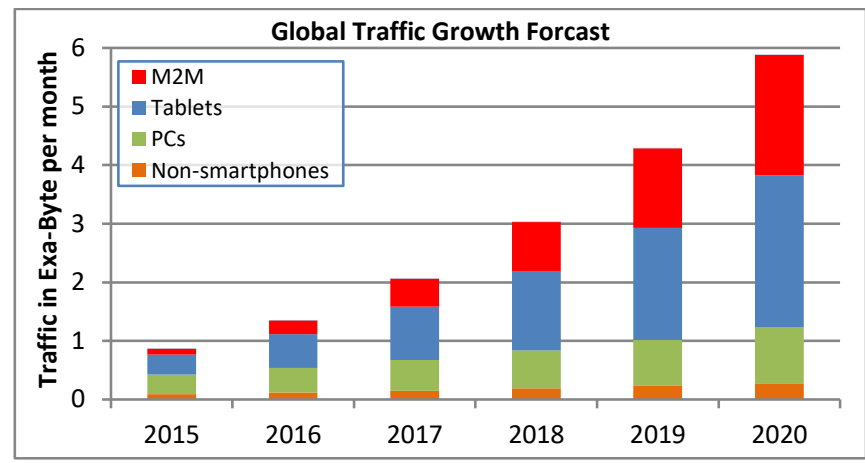

Fig.29. Global traffic forecast, showing M2M traffic growth rate [1].

\section{Standardisation in $\mathbf{m m}$-wave}

In 2001, the FCC allocated an additional $7 \mathrm{GHz}$ of bandwidth in the free-licensed $60 \mathrm{GHz}$ band from 57 to $64 \mathrm{GHz}$ for unlicensed use. This band has four channels available with $2.16 \mathrm{GHz}$ bandwidth. The propagation characteristics are different from the lower frequency due to severe atmospheric attenuation as a result of absorption by oxygen. The $60 \mathrm{GHz}$ band supports high speed data rates at short distances, with directional transmissions in LoS. These characteristics bring it into the category of Wireless Personal Area Networks (WPANs) [181].

A recent standard for path loss modelling in the mm-wave band has been released by $3 \mathrm{GPP}$, for the frequency spectrum above 6GHz (Release 14represented by the standards 3GPP-TR 38.900 and 3GPP-TR 38.901 published in [17][16]). These standards provide a study on channel modelling for mm-wave and describe a path loss model for LoS/NLoS transmission in 5G. The 3GPP has introduced three cases for 5G use; namely, enhanced Mobile Broadband (eMBB), mMTC and Ultra Reliable Low Latency Communications (URLLC). The eMBB focuses on very high data throughput mobile broadband services; mMTC considers supporting massive connectivity for machines in the IoT environment; and URLLC is required for applications and services that need stringent latency and high reliability requirements [182]. The ITU released the 5G network requirements in February 2017 [183] which describe the minimum requirements related to technical performance for IMT-2020 (5G) radio interface. The ITU has defined the required peak data rate (downlink/uplink), mobility support, spectral and energy efficiency requirements, and the projected device density to be supported per 5G areaa. The 5G Infrastructure Public Private Partnership (5G-PPP) is working towards a multi-tenancy vision with major stakeholders to unify the $5 \mathrm{G}$ digital markets and help multi networks to share infrastructures and equipment [184]. The 5G-PPP will deliver architectures, technologies and standards for ubiquitous 5G communication, with main focus on:providing higher wireless capacity of $1000 x$; energy saving per service in the RAN of $90 \%$; facilitating UDN connection to over 7 trillion devices; and creating ultra-reliable connectivity for internet service provision [184][185].

Mm-wave has also been proposed to accommodate the extreme bandwidth demands in Vehicle-to-Vehicle (V2V) communications, to support future autonomous vehicles needed to improve environmental awareness and safety [186]. The 5G-PPP Automotive Working Group [187] is working on vehicular communications to achieve safety and awareness in the V2V environment. See through obstacles and bird's eye views of intersections aredescribed in their " $5 \mathrm{G}$ Automotive Vision" white paper in [188]. The IEEE released the IEEE 802.11p standard [189] which is an amendment to the WiFi standard to support vehicular communications in the $5.9 \mathrm{GHz}$ band. But with high data volumes predicted from V2V links, it seems reasonable to exploit the IEEE 802.11ad standard for mm-wave V2V communications [190]. Future autonomous vehicles use forecasts around $1000 \mathrm{~GB}$ of data per hour generated from driving a vehicle, with data rates of $750 \mathrm{Mbit} / \mathrm{s}$. This demonstrates the limitations of current wireless technologies to cope with the huge future volumes of automotive data, and justifies the need to extend the wireless network operation to the mm-wave [190][191]. In [186] a novel framework has been proposed based on matching theory and swarm intelligence to pair V2V and optimise transmission and reception beamwidths. The work has considered Channel State Information (CSI) and Queue State Information (QSI) during V2V link establishment A gain of $25 \%$ is achieved in reliability and delay in the dense V2V environment and a $50 \%$ gain in active $\mathrm{V} 2 \mathrm{~V}$ links.

Furthermore, the European Telecommunications Standards Institute (ETSI) has established a technical committee focusing on providing M2M connectivity. There are two reference terminologies for M2M: the 3GPP - $\mathrm{mMTC}$ [182] and the IEEE-M2M standard: IEEE 802.16p [192], which include enhancements to support a M2M service. IEEE-M2M and 
3GPP - mMTC schemes complement to ETSI-M2M service. Therefore, it is possible to combine the M2M service by ETSI with any M2M/mMTC from IEEE or 3GPP, which results in a cellular-centric M2M architecture [193]. Additionally, to avoid market dispersion and reduce arbitrary standardisations [194], the "oneM2M" Partnership Project was founded in 2012 [195] The focus was to develop "one" standard for M2M specification and to consolidate M2M standard activities into a unified framework, known as oneM2M [19]. OneM2M is a global initiative to establish a unified M2M service platform [196] in order to achieve interoperability between M2M applications and the 5G network service.

In this context, many standards have emerged from different international organizations for the purpose of exploiting the mm-wave free band to achieve high speed data including IEEE 802.11ad, IEEE 802.15.3 and ECMA-387. These are discussed in the following subsections:

\subsection{IEEE 802.11ad Standard}

In IEEE 802.11ad, the task group has released the first draft to addressh the characteristics of $60 \mathrm{GHz}$ wireless communications. Amendments have been made to the 802.11 physical layer and MAC layer to support multi-gigabit wireless applications in the $60 \mathrm{GHz}$ band. Where two transmissions are specified in the standard, these are Orthogonal Frequency Division Multiplexing (OFDM) and Single Carrier (SC) transmission. OFDM is used when high performance applications are required. The low complexity SC is used for control information and low data rate requirements [52]. One key advantage of 802.11ad over other standards is that it builds on existing Wi-Fi standards in the $2.4 / 5 \mathrm{GHz}$ bands.

The idea behind the 802.11 amendment is backward compatibility.The 802.11 ad will be compatible in the MAC and Data Link layers with its predecessors. The only difference is in the physical layer (PHY) characteristics. Therefore, future handsets could have three transceivers: $2.4 \mathrm{GHz}$ for general use, $5 \mathrm{GHz}$ for higher speed applications, and $60 \mathrm{GHz}$ for ultra-high-speed data within very short distances [197].

\subsection{IEEE 802.15.3c Standard}

IEEE 802.15.3c defines PHY and MAC layers for mm-wave WPAN. In this standard, the MAC implements a random channel access and time division multiple access approach to support quasi-sectorisation as a result of highly directional antennas. This standard is also an amendment [181][198] to the existing IEEE802.15.3 MAC to support PHY at the $60 \mathrm{GHz}$ mm-wave band. For instance, techniques such as frame aggregation and block acknowledgment are introduced to reduce MAC overhead. IEEE $802.15 .3 \mathrm{c}$ is also used for an ad-hoc communication system for devices to communicate with each other in a "piconet" with a radius of around 10m [199]. The transmission can operate either in directional mode or in quasi-omnidirectional mode, in which a highly directional array is supported to generate the pattern.

\subsection{ECMA- 387}

In parallel with IEEE standardisation, a technology group and electronics company called ECMA International has released their own standard, ECMA-387. This standard specifies PHY, MAC, and a High-Definition Multimedia Interface (HDMI) protocol adaptation layer (PAL) for $60 \mathrm{GHz}$ wireless networks [200]. It is used for flexible and heterogeneous WPANs to provide multi-gigabit data rate services. The heterogeneous networks (HetNets) operating by ECMA can coexist, interoperate and can operate independently. Thus, this standard supports a wide range of implementations and applications from low data rate transfer at short distances, utilised for handheld devices, to high data rates used for multimedia streaming at longer distances, when adaptive antennas are used [201].

\section{Conclusion}

Mm-wave communications have proven to be a promising candidate for $5 \mathrm{G}$ mobile networks due to their potential to support an extreme capacity increase over a LTE network. In this paper, we have carried out a survey of the mm-wave band as a candidate bandwidth for $5 \mathrm{G}$ communications, which focus on potential network architectures that exploit mmwave unique propagation characteristics such as HOS and DBS architecture. The challenges of this band have been identified and discussed. A radical new design to the mobile networks architecture is needed, in order to address these challenges. Such a radical design may include BS level (equipment) as well as network level (core and backhaul) 
considerations. The most recent solutions developed in this field have been highlighted in this survey and their initiatives have been discussed. This survey has also investigated the role of mm-wave in $5 \mathrm{G}$ in areas such as outdoor, indoor, and as a backhaul solution, and has discussed how massive MIMO with hybrid beamforming are used in order to provide reliable services in $5 \mathrm{G}$.

Relevant and open issues related to mm-wave communications have been presented and discussed, this includes interference coordination and optimising handoff in a UDN environment, providing MIMO streams in the LoS environment, new waveform considerations to replace OFDM, and supporting massive MTC traffic. A DBS network architecture has been introduced in this band in order to address some issues in the UDN environment, including higher handoff rates and increased ICI, and to provide an alternative option for network densification. Furthermore, the current standards operating at the mm-wave band have been introduced and discussed. Recent contributions through empirical measurements, analysis, and software simulation have demonstrated that mm-wave is a viable option for next generation wireless systems for indoor and outdoor mobile access as well as backhaul links, to provide "massive" capacity and connectivity to users and enormous number of devices and machines.

\section{Acknowledgment}

This work is fully sponsored by the Ministry of Higher Education and Scientific Research in Iraq (MOHESR) University of Anbar.

\section{References}

[1] Cisco, “Cisco Visual Networking Index : Global Mobile Data Traffic Forecast Update , 2015-2020,” White Pap., pp. 1-39, 2016.

[2] W. Roh et al., "Millimeter-Wave Beamforming as an Enabling Technology for 5G Cellular Communications : Theoretical Feasibility and Prototype Results," IEEE Commun. Mag., vol. 52, no. 2, pp. 106-113, 2014.

[3] F. Boccardi, R. W. H. Jr., A. Lozano, T. L. Marzetta, and P. Popovski, "Five Disruptive Technology Directions for 5G," IEEE Commun. Mag., vol. 52, no. 2, pp. 74-80, 2014.

[4] F. Khan and Z. Pi, “An Introduction to Millimeter-Wave Mobile Broadband Systems,” IEEE Commun. Mag., vol. 59, no. 6, pp. 101-107, 2011 .

[5] N. Al-Falahy and O. Alani, “Technologies for 5G Networks: Challenges and Opportunities,” IEEE IT Prof., vol. 19 , no. 1, pp. $12-20,2017$.

[6] Federal Communications Commission (FCC), "Radio Spectrum Allocation,” 2018. [Online]. Available: www.fcc.gov/engineeringtechnology/policy-and-rules-division/general/radio-spectrum-allocation. [Accessed: 06-Apr-2018].

[7] International Telecommunication Union (ITU), "World Radiocommunication Conferences (WRC)," 2018. [Online]. Available: https://www.itu.int/en/ITU-R/conferences/wrc. [Accessed: 06-Apr-2018].

[8] Federal Communications Commission (FCC), "Use of Spectrum Bands Above 24 GHz for Mobile Radio Services," Technical Report, no. GN Docket No. 14-177, pp. 1-137, 2017.

[9] Ofcom, Update on $5 G$ spectrum in the UK, no. February. 2017, pp. 1-19.

[10] Q. Li, H. Niu, A. Papathanassiou, and G. Wu, "5G Network Capacity, key elements and technologies," IEEE Veh. Technol. Mag., vol. 9, no. 1, pp. 71-78, 2014.

[11] S. G. Larew, T. A. Thomas, M. Cudak, and A. Ghosh, "Air Interface Design and Ray Tracing Study for 5G Millimeter Wave Communications," in IEEE Globecom Workshops, 2013, pp. 117-122.

[12] T. S. Rappaport et al., "Millimeter Wave Mobile Communications for 5G Cellular: It Will Work !," IEEE Access, vol. 1, no. DOI: 10.1109/ACCESS.2013.2260813, pp. 335-349, 2013.

[13] S. Rajagopal, S. Abu-Surra, and M. Malmirchegini, "Channel Feasibility for Outdoor Non-Line-of-Sight mmWave Mobile Communication," in IEEE Vehicular Technology Conference (VTC Fall), 2012, pp. 1-6.

[14] W. Keusgen, R. J. Weiler, M. Peter, M. Wisotzki, and B. Goktepe, "Propagation measurements and simulations for millimeter-wave mobile access in a busy urban environment," in 39th International Conference on Infrared, Millimeter, and Terahertz waves (IRMMW-THz), 2014, pp. 1-3.

[15] T. S. Rappaport, F. Gutierrez, E. Ben-dor, J. N. Murdock, Y. Qiao, and J. I. Tamir, "Broadband Millimeter-Wave Propagation Measurements and Models Using Adaptive-Beam Antennas for Outdoor Urban Cellular Communications," IEEE Trans. Antennas Propag., vol. 61 , no. 4 , pp. 1850-1859, 2013.

[16] 3GPP Technical Report 38.901, “Technical Specification Group Radio Access Network; Study on channel model for frequencies from 0.5 to $100 \mathrm{GHz}, " 2017$.

[17] 3GPP Technical Report 38.900, "Technical Specification Group Radio Access Network; Study on channel model for frequency spectrum above 6 GHz." pp. 1-85, 2017.

[18] M. Taranetz, S. Schwarz, and M. Rupp, The Vienna LTE-Advanced Simulators, Up and Downlink, Link and System Level Simulation. Springer Singapore, 2016.

[19] N. Al-Falahy and O. Alani, "Improved Capacity and Fairness of Massive Machine Type Communications in Millimetre Wave 5G Network," Computers, vol. 7, no. DOI:10.3390/computers7010016, pp. 1-19, 2018.

[20] T. S. Rappaport, J. N. Murdock, and F. Gutierrez, "State of the art in 60-GHz integrated circuits and systems for wireless communications," IEEE Proc., vol. 99, no. 8, pp. 1390-1436, 2011.

[21] E. Semaan, F. Harrysson, A. Furusk, and H. Asplund, "Outdoor-to-Indoor Coverage in High Frequency Bands," in Globecom Workshop Mobile Communications in Higher Frequency Bands, 2014, pp. 393-398.

[22] A. Gupta and R. K. Jha, "A Survey of 5G Network: Architecture and Emerging Technologies," IEEE Access, no. DOI 
10.1109/ACCESS.2015.2461602, pp. 1-27, 2015.

[23] M. Marcus and B. Pattan, "Millimeter Wave Propagation : Spectrum Management Implications," IEEE Microw. Mag., vol. 6, no. 2 , 2005.

[24] C. R. Anderson et al., "In-building wideband multipath characteristics at 2.5 and $60 \mathrm{GHz}$," in Proceedings IEEE 56th Vehicular Technology Conference, 2002, pp. 97-101.

[25] K. C. Allen, N. DeMinco, J.R. Hoffman, Y. Lo, and B. P. P, "Building penetration loss at $900 \mathrm{MHz}, 11.4 \mathrm{GHz}$, and $28.8 \mathrm{GHz}$," NTIA Rep. 94-306, no. May, 1994.

[26] H. Zhao et al., " $28 \mathrm{GHz}$ millimeter wave cellular communication measurements for reflection and penetration loss in and around buildings in New York city," in IEEE International Conference on Communications, 2013, pp. 5163-5167.

[27] A. V. Alejos, M. G. Sanchez, and I. Cuinas, "Measurement and analysis of propagation mechanisms at $40 \mathrm{GHz}$ : Viability of site shielding forced by obstacles," IEEE Trans. Veh. Technol., vol. 57, no. 6, pp. 3369-3380, 2008.

[28] ITU-R P.838-2, "Specific attenuation model for rain for use in prediction methods," Recomm. ITU-R P.638-3, pp. 1-5, 2005

[29] P. Kántor and J. Bitó, "Influence of Climate Variability on Performance of Millimeter Wave Cellular 5th Generation Wireless Systems in Consequence of Precipitation," in The 8th European Conference on Antennas and Propagation (EuCAP), 2014, pp. 517-521.

[30] C. Dehos, J. L. González, A. De Domenico, D. Kténas, and L. Dussopt, "Millimeter-Wave Access and Backhauling: The Solution to the Exponential Data Traffic Increase in 5G Mobile Communications Systems ?," IEEE Commun. Mag., vol. 52, no. 9, pp. 88-95, 2014.

[31] S. Akoum, O. El Ayach, and R. W. Heath, "Coverage and capacity in mmWave cellular systems," in (ASILOMAR) Conference on Signals, Systems and Computers, 2012, pp. 688-692.

[32] C. Kourogiorgas, S. Sagkriotis, and A. D. Panagopoulos, "Coverage and Outage Capacity Evaluation in 5G Millimeter Wave Cellular Systems : Impact of Rain Attenuation," in 9th European Conference on Antennas and Propagation (EuCAP), 2015, pp. 1-5.

[33] A. Ghosh et al., "Millimeter-Wave Enhanced Local Area Systems : A High-Data-Rate Approach for Future Wireless Networks," IEEE J. Sel. Areas Commun., vol. 32, no. 6, pp. 1152-1163, 2014.

[34] A. Al-Hourani, S. Chandrasekharan, and S. Kandeepan, "Path loss study for millimeter wave device-to-device communications in urban environment," in IEEE International Conference on Communications Workshops (ICC), 2014, pp. 102-107.

[35] J. Bae, H.-S. Park, S. J. Lee, Y. S. Choi, J. S. Kim, and M. Y. Chung, "System coverage of Mm wave based 5G mobile communication system," in Asia-Pacific Conference on Communications (APCC), 2014, pp. 372-375.

[36] Qualcomm, "The 1000x Mobile Data Challenge," White paper, no. November, pp. 1-38, 2013.

[37] S. R. Lamas and D. Gonz, "Indoor Planning Optimization of Ultra-dense Cellular Networks at High Carrier Frequencies," in IEEE Wireless Communications and Networking Conference (WCNC) - Workshops - 5G Architecture, 2015, pp. 23-28.

[38] S. Nie, G. R. M. Jr, S. Sun, and T. S. Rappaport, "72 GHz Millimeter Wave Indoor Measurements for Wireless and Backhaul Communications," in IEEE 24th International Symposium on Personal, Indoor and Mobile Radio Communications: Mobile and Wireless Networks, 2013, pp. 2429-2433.

[39] C. Gustafson, K. Haneda, S. Wyne, S. Member, and F. Tufvesson, "On mm-Wave Multipath Clustering and Channel Modeling," IEEE Trans. Antennas Propag., vol. 62, no. 3, pp. 1445-1455, 2014.

[40] B. Neekzad, K. Sayrafian-Pour, J. Perez, and J. S. Baras, "Comparison of Ray Tracing Simulations and Millimeter Wave Channel Sounding Measurements," in IEEE 18th International Symposium on Personal, Indoor and Mobile Radio Communications, 2007, pp. 1-5.

[41] D. Du, J. Zhang, C. Pan, and C. Zhang, "Cluster characteristics of wideband 3D MIMO channels in outdoor-to-indoor scenario at 3.5 GHz," in IEEE Vehicular Technology Conference, 2015, pp. 3-8.

[42] S. Hur, Y. J. Cho, J. Lee, N. G. Kang, J. Park, and H. Benn, "Synchronous channel sounder using horn antenna and indoor measurements on 28 GHz," in IEEE International Black Sea Conference on Communications and Networking, BlackSeaCom 2014, 2014, pp. 83-87.

[43] C. Larsson, F. Harrysson, B. Olsson, and J. Berg, "An Outdoor-to-Indoor Propagation Scenario at 28 GHz," in The 8th European Conference on Antennas and Propagation (EuCAP), 2014, pp. 3985-3988.

[44] M. T. Martinez-Ingles, C. Sanchis-Borras, J. M. Molina-Garcia-Pardo, J. V. Rodríguez, and L. Juan-Llácer, "Experimental Evaluation of an Indoor MIMO-OFDM System at $60 \mathrm{GHz}$ Based on the IEEE802.15.3c Standard," IEEE ANTENNAS Wirel. Propag., vol. 12, pp. 15621565,2013

[45] L. Rakotondrainibe, Y. Kokar, G. Zaharia, and G. El Zein, "Millimeter-wave system for high data rate indoor communications," in International Symposium on Signals, Circuits and Systems, 2009, pp. 1-4.

[46] A. Maltsev et al., "Characteristics of indoor millimeter-wave channel at $60 \mathrm{GHz}$ in application to perspective WLAN system," in Proceedings of the Fourth European Conference on Antennas and Propagation (EuCAP), 2010, pp. 1-5.

[47] Z. Wen and Hongwei Kong, "mmWave MIMO Channel Sounding for 5G," in 1st International Conference on $5 G$ for Ubiquitous Connectivity (5GU), 2014, pp. 192-197.

[48] B. Neekzad, "Space-time Behavior of Millimeter Wave Channel and Directional Medium Access Control," University of Maryland, 2008.

[49] E. Torkildson, U. Madhow, and M. Rodwell, "Indoor millimeter wave MIMO: Feasibility and performance," IEEE Trans. Wirel. Commun., vol. 10, no. 12, pp. 4150-4160, 2011.

[50] Y. Choi and Y. Han, "Modeling and Analysis of Millimeter/Sub-millimeter Wave Indoor Communications for Multi-gigabit Wireless Transmission," in 39th International Conference on Infrared, Millimeter, and Terahertz waves (IRMMW-THz), 2014, pp. 1-2.

[51] G. Li, T. Irnich, and C. Shi, "Coordination context - based spectrum sharing for 5G millimeter - wave networks - Invited Paper -," in 9th International Conference on Cognitive Radio Oriented Wireless Networks and Communications (CROWNCOM), 2014, pp. 32-38.

[52] X. Zhu, A. Doufexi, and T. Kocak, "A Performance Evaluation of $60 \mathrm{GHz}$ MIMO Systems for IEEE 802 . 11ad WPANs," IEEE Proceeding Indoor Mob. Radio Commun., pp. 950-954, 2011.

[53] B. Neekzad, K. Sayrafian-Pour, and J. S. Baras, "Clustering Characteristics of Millimeter Wave Indoor Channels," in 2008 IEEE Wireless Communications and Networking Conference, 2008, pp. 1217-1222.

[54] L. Wang, J. Chen, X. Wei, J. Cui, and B. Zheng, "First-order-reflection MIMO Channel Model for 60 GHz NLOS Indoor WLAN Systems," in IEEE International Conference on Communication Systems (ICCS), 2014, pp. 298-302.

[55] L. Liu and Y. Li, "DoA Estimation and Achievable Rate Analysis for 3D Millimeter Wave Massive MIMO Systems," in IEEE 15th International Workshop on Signal Processing Advances in Wireless Communications (SPAWC), 2014, pp. 6-10.

[56] Lili Wei, R. Q. Y. Hu, Y. Qian, and G. Wu, "Key elements to enable millimeter wave communications for 5G wireless systems," IEEE Wirel. Commun., vol. 21, no. 6, pp. 136-143, 2014. 
[57] S. Rajagopal, S. Abu-Surra, and F. Khan, "Antenna Array Design for Multi-Gbps mmWave Mobile Broadband Communication," in IEEE Global Telecommunications Conference - GLOBECOM, 2011, pp. 1-6.

[58] A. I. Sulyman et al., "Radio Propagation Path Loss Models for 5G Cellular Networks in the 28 GHz and 38 GHz Millimeter-Wave Bands," IEEE Commun. Mag., vol. 52, no. 9, pp. 78-86, 2014.

[59] G. MacCartney, J. Zhang, S. Nie, and T. Rappaport, "Path Loss Models for 5G Millimeter Wave Propagation Channels in Urban Microcells," in Globecom - Wireless Communications Symposium, 2013, pp. 3948-3953.

[60] G. R. M. Jr and T. S. Rappaport, "73 GHz Millimeter Wave Propagation Measurements for Outdoor Urban Mobile and Backhaul Communications in New York City," in IEEE ICC, 2014, pp. 4862-4867.

[61] C. Sheldon, E. Torkildson, M. Seo, C. P. Yue, M. Rodwell, and U. Madhow, "Spatial multiplexing over a line-of-sight millimeter-wave MIMO link: A two-channel hardware demonstration at $1.2 \mathrm{Gbps}$ over $41 \mathrm{~m}$ range," in 1st European Conference on Wireless Technology, 2008, pp. 198-201.

[62] N. Al-falahy and O. Alani, "The Impact of Base Station Antennas Configuration on the Performance of Millimetre Wave 5G Networks," in The ninth International Conference on Ubiquitous and Future Networks (ICUFN ), 2017, pp. 636-641.

[63] V. Raghavan, A. Partyka, L. Akhoondzadeh-Asl, M. A. Tassoudji, O. H. Koymen, and J. Sanelli, "Millimeter Wave Channel Measurements and Implications for PHY Layer Design,” IEEE Trans. Antennas Propag., vol. 65, no. 12, pp. 6521-6533, 2017.

[64] C. U. Bas et al., "Outdoor to Indoor Penetration Loss at 28 GHz for Fixed Wireless Access," Computing Research Repository, CoRR-arXiv, no. arXiv:1711.00168v1 [cs.IT] 1 Nov 2017, pp. 1-6, 2017.

[65] J. Medbo, D. Sundman, H. Asplund, and S. Dwivedi, "Wireless Urban Propagation Measurements at 2.44, 5.8, 14.8 \& 58.68 GHz," in 32nd General Assembly and Scientific Symposium of the International Union of Radio Science (URSI GASS), 2017, pp. 2-5.

[66] S. L. Cotton, W. G. Scanlon, and B. K. Madahar, "Millimeter-Wave Soldier-to-Soldier Communications for Covert Battlefield Operations," IEEE Commun. Mag., vol. 47, no. 10, pp. 72-81, 2009.

[67] M. R. Akdeniz, S. Rangan, and E. Erkip, "Millimeter wave picocellular system evaluation for urban deployments," in IEEE Globecom Workshops (GC Wkshps), 2013, pp. 105-110.

[68] S. Piersanti, L. A. Annoni, and D. Cassioli, "Millimeter waves channel measurements and path loss models," in IEEE International Conference on Communications (ICC), 2012, pp. 4552-4556.

[69] H. Xu, T. S. Rappaport, R. J. Boyle, and J. H. Schaffner, "Measurements and Models for 38-GHz Point-to-Multipoint Radiowave Propagation," IEEE J. Sel. Areas Commun., vol. 18, no. 3, pp. 310-321, 2000.

[70] T. S. Rappaport, E. Ben-Dor, J. N. Murdock, and Y. Qiao, “38 GHz and $60 \mathrm{GHz}$ angle-dependent propagation for cellular \& peer-to-peer wireless communications," in IEEE International Conference on Communications (ICC), 2012, pp. 4568-4573.

[71] M. Samimi et al., "28 GHz Angle of Arrival and Angle of Departure Analysis for Outdoor Cellular Communications Using Steerable Beam Antennas in New York City," in IEEE 77th Vehicular Technology Conference (VTC Spring), 2013, pp. 1-6.

[72] J. N. Murdock, E. Ben-Dor, Y. Qiao, J. I. Tamir, and T. S. Rappaport, "A 38 GHz cellular outage study for an urban outdoor campus environment," in IEEE Wireless Communications and Networking Conference (WCNC), 2012, pp. 3085-3090.

[73] P. K. Agyapong, M. Iwamura, D. Staehle, W. Kiess, and A. Benjebbour, "Design Considerations for a 5G Network Architecture," IEEE Commun. Mag., vol. 52, no. 11, pp. 65-75, 2014.

[74] T. Bai and Heath Jr. Robert W, "Coverage and Rate Analysis for Millimeter Wave Cellular Networks,” http://arxiv.org/abs/1402.6430v2, no. 1218338, pp. 1-33, 2014.

[75] T. Bai and R. W. Heath, "Coverage analysis for millimeter wave cellular networks with blockage effects," in IEEE Global Conference on Signal and Information Processing, Global SIP Proceedings, 2013, pp. 727-730.

[76] W. EI-Beainol, A. M. EI-Hajj, and Z. Dawy, “On Radio Network Planning for Next Generation 5G Networks: A Case Study," in International Conference on Communications, Signal Processing, and their Applications (ICCSPA), 2015, pp. 1-6.

[77] R. Taori and A. Sridharan, "Point-to-multipoint in-band mmwave backhaul for 5G networks," IEEE Commun. Mag., vol. 53, no. 1, pp. 195-201, 2015.

[78] X. Su and K. Chang, "A comparative study on wireless backhaul solutions for beyond 4G network," in The International Conference on Information Networking 2013 (ICOIN), 2013, pp. 505-510.

[79] F. Gutierrez, "Millimeter-wave and sub-terahertz on-chip antennas, arrays, propagation, and radiation pattern measurements," The University of Texas at Austin, 2013.

[80] H. Peng, T. Yamamoto, and Y. Suegara, "Extended User / Control Plane Architectures for Tightly Coupled LTE / WiGig Interworking in Millimeter-wave Heterogeneous Networks," in IEEE Wireless Communications and Networking Conference (WCNC): - Track 3: Mobile and Wireless Networks, 2015, pp. 1548-1553.

[81] M. N. Kulkarni, S. Singh, and J. G. Andrews, "Coverage and Rate Trends in Dense Urban mmWave Cellular Networks," in Globecom 2014 - Wireless Communications Symposium Coverage, 2014, pp. 3809-3814.

[82] G. MacCartney, T. Rappaport, M. Samimi, and S. Sun, "Millimeter Wave Omnidirectional Path Loss Data for Small Cell 5G Channel Modeling," IEEE Access, vol. 10.1109/AC, pp. 1-6, 2015.

[83] R. J. Weiler, M. Peter, W. Keusgen, and M. Wisotzki, "Measuring the Busy Urban 60 GHz Outdoor Access Radio Channel," in IEEE International Conference on Ultra-Wideband, ICUWB, 2014, pp. 166-170.

[84] M. N. Kulkarni, T. A. Thomas, F. W. Vook, A. Ghosh, and E. Visotsky, "Coverage and Rate Trends in Moderate and High Bandwidth 5G Networks," in Globecom Workshop, 2014, pp. 422-426.

[85] Z. Pi and F. Khan, "A millimeter-wave massive MIMO system for next generation mobile broadband," in Asilomar Conference on Signals, Systems and Computers, 2012, pp. 693-698.

[86] A. Heights, T. A. Thomas, and F. W. Vook, "System Level Modeling and Performance of an Outdoor mmWave Local Area Access System," in IEEE 25th International Symposium on Personal, Indoor and Mobile Radio Communications System, 2014, pp. 108-112.

[87] N. Bhushan et al., "Network Densification: The Dominant Theme for Wireless Evolution into 5G," IEEE Commun. Mag., vol. 52, no. 2, pp. 82-89, 2014.

[88] N. Al-falahy and O. Alani, "Design Considerations of Ultra Dense 5G Network in Millimetre Wave Band," in The ninth International Conference on Ubiquitous and Future Networks (ICUFN ), 2017, pp. 141-146.

[89] N. Al-Falahy and O. Alani, "The Impact of Higher Order Sectorisation on the Performance of Millimetre Wave 5G Network," in The 10th International Conference on Next Generation Mobile Applications, Security and Technologies (NGMAST2016), 2016, pp. 1-5. 
[90] ATDI, “ICS Designer by ATDI.” [Online]. Available: www.atdi.co.uk/software/radio-planning/ics-designer. [Accessed: 04-Aug-2017].

[91] D. Erik, S. Parkvall, and S. Johan, 4G LTE/LTE-Advanced for Mobile Broadband, First Edit. Oxford,: Elsevier Ltd., 2011.

[92] W. R. Highsmith, "An investigation into distributed base station design for LMDS systems," in Proceedings IEEE SoutheastCon 2002 (Cat. No.02CH37283), 2002, pp. 162-165.

[93] H. Q. Ngo, A. Ashikhmin, H. Yang, E. G. Larsson, and T. L. Marzetta, "Cell-Free Massive MIMO: Uniformly great service for everyone," in IEEE Workshop on Signal Processing Advances in Wireless Communications, SPAWC, 2015, pp. 201-205.

[94] H. Q. Ngo, A. Ashikhmin, H. Yang, E. G. Larsson, and T. L. Marzetta, "Cell-Free Massive MIMO Versus Small Cells," IEEE Trans. Wirel. Commun., vol. 16, no. 3, pp. 1834-1850, 2017.

[95] E. Yaacoub and Z. Dawy, Resource Allocation in Uplink OFDMA Wireless Systems: Optimal Solutions and Practical Implementations. Wiley-IEEE Press, 2012.

[96] W. Choi, J. G. Andrews, and C. Yi, "Capacity of multicellular distributed antenna networks," in International Conference on Wireless Networks, Communications and Mobile Computing, 2005, pp. 1337-1342.

[97] J. Zhang and J. G. Andrews, “Distributed antenna systems with randomness,” IEEE Trans. Wirel. Commun., vol. 7, no. 9, pp. 3636-3646, 2008.

[98] T. E. Bogale and L. B. Le, "Massive MIMO and mmWave for 5G Wireless HetNet Potential Benefits and Challenges," IEEE Veh. Technol. Mag., vol. 11, no. 1, pp. 64-75, 2016.

[99] S. Mandal and S. Gauni, "Energy Efficiency Of Single Cell And Multi Cell Massive MIMO System using MMSE Estimation," in International Conference on Next gen Electronic Technologies: Silicon to Software (ICNETS2), 2017, pp. 66-70.

[100] H. Xie, F. Gao, S. Jin, J. Fang, and Y.-C. Liang, "Channel Estimation for TDD/FDD Massive MIMO Systems with Channel Covariance Computing,” arXiv Comput. Sci. Inf. Theory, no. arXiv:1710.00704v1, pp. 1-30, 2017.

[101] C. Lin, C. Rowell, S. Han, Z. Xu, G. Li, and Z. Pan, “Toward Green and Soft : A 5G Perspective,” IEEE Commun. Mag., vol. 52, no. 2, pp. 66-73, 2014

[102] C. Cox, An Introduction to LTE, LTE-Advanced, SAE And 4G Mobile Communications, First Edit. Sussex: John Wiley \& Sons, Ltd., 2012.

[103] S. Noh, M. D. Zoltowski, Y. Sung, and D. J. Love, "Pilot Beam Pattern Design for Channel Estimation in Massive MIMO Systems," IEEE J. Sel. Top. Signal Process., vol. 8, no. 5, pp. 787-801, 2014.

[104] Z. Li, S. Han, S. Sangodoyin, R. Wang, and A. F. Molisch, "Joint Optimization of Hybrid Beamforming for Multi-User Massive MIMO Downlink," IEEE Trans. Wirel. Commun., no. DOI 10.1109/TWC.2018.2808523, pp. 1-14, 2018.

[105] J. Choi, "Massive MIMO With Joint Power Control," IEEE Wirel. Commun. Lett., vol. 3, no. 4, pp. 329-332, Aug. 2014.

[106] J. Zhu, S. Member, R. Schober, V. K. Bhargava, and L. Fellow, "Secure Transmission in Multicell Massive MIMO Systems," IEEE Trans. Wirel. Commun., vol. 13, no. 9, pp. 4766-4781, 2014.

[107] E. Uihetkangas, K. Pajukoski, and E. Tiirola, "On the Flexible 5G Dense Deployment Air Interface for Mobile Broadband," in 1st International Conference on $5 G$ for Ubiquitous Connectivity (5GU), 2014, pp. 57-61.

[108] M. Di Renzo, H. Haas, A. Ghrayeb, S. Sugiura, and L. Hanzo, "Spatial Modulation for Generalized MIMO: Challenges, Opportunities, and Implementation," Proc. IEEE, vol. 102, no. 1, pp. 56-103, Jan. 2013

[109] D. Zhu, B. Li, and P. Liang, "A Novel Hybrid Beamforming Algorithm with Unified Analog Beamforming by Subspace Construction Based on Partial CSI for Massive MIMO-OFDM Systems," IEEE Trans. Commun., vol. 65, no. 2, pp. 594-607, 2017.

[110] Y. Kim, H. Lee, J. Oh, J. Lee, W. Roh, and K. Cheun, "Feasibility of Mobile Cellular Communications at Millimeter Wave Frequency," IEEE J. Sel. Top. Signal Process., vol. 10, no. 3, pp. 589-599, 2016.

[111] L. Lu, G. Y. Li, A. L. Swindlehurst, A. Ashikhmin, and R. Zhang, “An Overview of Massive MIMO : Benefits and Challenges,” IEEE J. Sel. Top. Signal Process., vol. 8, no. 5, pp. 742-758, 2014.

[112] T. L. Marzetta, "Massive MIMO: An introduction,” Bell Labs Technical Journal, vol. 20, pp. 11-12, 2015.

[113] A. L. Swindlehurst, E. Ayanoglu, P. Heydari, and F. Capolino, "Millimeter-Wave Massive MIMO : The Next Wireless Revolution ?," IEEE Commun. Mag., vol. 52, no. 9, pp. 56-62, 2014.

[114] X. Jiang and F. Kaltenberger, "Channel reciprocity calibration in TDD hybrid beamforming massive MIMO systems," IEEE J. Sel. Top. Signal Process., no. DOI 10.1109/JSTSP.2018.2819118, pp. 1-10, 2018.

[115] R. W. Heath Jr., N. González-Prelcic, S. Rangan, W. Roh, and A. M. Sayeed, “An overview of signal processing techniques for millimeter wave MIMO systems," IEEE J. Sel. Top. Signal Process., vol. 10, no. 3, pp. 436-453, 2016

[116] Z. Pi and F. Khan, "System design and network architecture for a millimeter-wave mobile broadband (MMB) system," in 2011 34th IEEE Sarnoff Symposium, SARNOFF 2011, 2011, pp. 1-6.

[117] E. Perahia, C. Cordeiro, M. Park, and L. L. Yang, "IEEE 802.11ad: Defining the Next Generation Multi-Gbps Wi-Fi," in IEEE Consumer Communications and Networking Conference, 2010, pp. 3-7.

[118] P. Mogensen et al., "Centimeter-wave concept for 5G ultra-dense small cells," in IEEE 79th Vehicular Technology Conference (VTC Spring), 2014, no. 3.

[119] C. Kim, J. Son, T. Kim, and J. Seol, "On the Hybrid Beamforming with Shared Array Antenna for mmWave MIMO-OFDM Systems," in IEEE WCNC'14 Track 1 (PHY and Fundamentals), 2014, vol. 1, pp. 335-340.

[120] S. Han, C.-L. I, Z. Xu, and C. Rowell, "Large-Scale Antenna Systems with Hybrid Analog and Digital Beamforming for Millimeter Wave 5G,” IEEE Commun. Mag., vol. 53, no. 1, pp. 186-194, 2015.

[121] D. Castanheira, P. Lopes, A. Silva, and A. Gameiro, "Hybrid Beamforming Designs for Massive MIMO Millimeter-Wave Heterogeneous Systems," IEEE Access, vol. 5, no. DOI: 10.1109/ACCESS.2017.2762361, pp. 21806-21817, 2017.

[122] F. Sohrabi and W. Yu, "Hybrid Analog and Digital Beamforming for mmWave OFDM Large-Scale Antenna Arrays," IEEE J. Sel. Areas Commun., vol. 35, no. 7, pp. 1432-1443, 2017.

[123] R. Magueta, D. Castanheira, A. Silva, R. Dinis, and A. Gameiro, "Hybrid Iterative Space-Time Equalization for Multi-User mmW Massive MIMO Systems," IEEE Trans. Commun., vol. 65, no. 2, pp. 608-620, 2017.

[124] S. Park, A. Alkhateeb, and R. W. Heath, "Dynamic Subarrays for Hybrid Precoding in Wideband mmWave MIMO Systems," IEEE Trans. Wirel. Commun., vol. 16, no. 5, pp. 2907-2920, 2017.

[125] J. Karjalainen, M. Nekovee, H. Benn, W. Kim, J. Park, and H. Sungsoo, "Challenges and Opportunities of mm-Wave Communication in 5G Networks," in 9th International Conference on Cogmitive Radio Oriented Networks (CROWNCOM), 2014, pp. 372-376. 
[126] S. Sun, T. S. Rappaport, R. W. Heath, A. Nix, and S. Rangan, "MIMO for Millimeter-Wave Wireless Communications : Beamforming , Spatial Multiplexing, or Both ?," IEEE Commun. Mag., vol. 52, no. 12, pp. 110-121, 2014.

[127] L. Sung, D. Park, and D.-H. Cho, "Limited Feedback Hybrid Beamforming Based on Dual Polarized Array Antenna," IEEE Commun. Lett. no. DOI 10.1109/LCOMM.2018.2817190, pp. 1-4, 2018.

[128] Y. S. Cho, J. Kim, W. Y. Yang, and C. G. Kang, MIMO-OFDM Wireless Communications with MATLAB. John Wiley \& Sons, Ltd., 2010.

[129] T. K. Lyu, "Capacity of multi-user MIMO systems with MMSE and ZF precoding," in IEEE INFOCOM, 2016, pp. 1083-1084.

[130] R. J. Weiler et al., "Enabling 5G backhaul and access with millimeter-waves," in IEEE European Conference on Networks and Communications (EuCNC), 2014, pp. 1-5.

[131] X. Ge, H. Cheng, M. Guizani, and T. Han, "5G wireless backhaul networks: challenges and research advances," IEEE Netw., vol. 28, no. 6, pp. 6-11, 2014.

[132] L. Dussopt, O. El Bouayadi, J. Alberto, Z. Luna, C. Dehos, and Y. Lamy, "Millimeter-Wave Antennas for Radio Access and Backhaul in 5G Heterogeneous Mobile Networks," in 9th European Conference on Antennas and Propagation (EuCAP), 2015, pp. 1-4.

[133] T. Wang and B. Huang, "Millimeter-wave Techniques for 5G Mobile Communications Systems : Challenges , Framework and Way Forward," in General Assembly and Scientific Symposium (URSI GASS), 2014, pp. 4-7.

[134] J. Wells, "Multigigabit wireless technology at $70 \mathrm{GHz}, 80 \mathrm{GHz}$ and $90 \mathrm{GHz}$," RF Des. Mag., no. May, pp. 50-58, 2006.

[135] B. Panzner, "In-band Wireless Backhaul for 5G Millimeter Wave Cellular Communications - Interactive Live Demo -," in IEEE Conference on Computer Communications Workshops (INFOCOM WKSHPS), 2015, pp. 21-22.

[136] V. Nikolikj and T. Janevski, "Profitability and Comparative Cost-Capacity Analysis of 5G Millimeter-Wave Systems," in 22nd Telecommunications Forum Conference (TELFOR), 2014, pp. 256-259.

[137] J. Qiao, "Enabling Millimeter Wave Communication for 5G Cellular Networks : MAC-layer Perspective," University of Waterloo, 2015.

[138] D. Wu, J. Wang, Y. Cai, and M. Guizani, "Millimeter-Wave Multimedia Communications : Challenges , Methodology , and Applications," IEEE Commun. Mag., vol. 53, no. 1, pp. 232-238, 2015.

[139] J. Qiao, X. Shen, J. W. Mark, Q. Shen, Y. He, and L. Lei, "Enabling Device-to-Device Communications in Millimeter-Wave 5G Cellular Networks," IEEE Commun. Mag., vol. 53, no. 1, pp. 209-215, 2015.

[140] A. Talukdar, M. Cudak, and A. Ghosh, "Handoff Rates for Millimeterwave 5G Systems," in IEEE 79th Vehicular Technology Conference (VTC Spring), 2014, pp. 1-5.

[141] T. Levanen, J. Pirskanen, and M. Valkama, "Radio Interface Design for Ultra-Low Latency Millimeter-Wave Communications in 5G Era," in Globecom Workshops (GC Wkshps), 2014, pp. 1420-1426.

[142] J. Kim, "Elements of Next-Generation Wireless Video Systems : Millimeter-Wave and Device-to-Device Algorithms,” UNIVERSITY OF SOUTHERN CALIFORNIA, 2014.

[143] W. Hong, K.-H. Baek, Y. Lee, Y. Kim, and S.-T. Ko, "Study and Prototyping of Practically Large-Scale mmWave Antenna Systems for 5G Cellular Devices," IEEE Commun. Mag., vol. 52, no. 9, pp. 63-69, 2014.

[144] W. Zhai, V. Miraftab, and M. Repeta, "Broadband Antenna Array with Low Cost PCB Substrate for 5G Millimeter Wave Applications," in Global Symposium On Millimeter Waves (GSMM), 2015, pp. 1-3.

[145] H. Zhou, "Phased array for millimeter-wave mobile handset," in IEEE Antennas and Propagation Society International Symposium, 2014, pp. 933-934.

[146] H. Mehrpouyan, M. Matthaiou, R. Wang, G. K. Karagiannidis, and Y. Hua, "Hybrid Millimeter-Wave Systems : A Novel Paradigm for HetNets," IEEE Commun. Mag., vol. 53, no. 1, pp. 216-221, 2015.

[147] T. Kim, I. Bang, and D. K. Sung, "Design Criteria on a mmWave-based Small Cell with Directional Antennas," in IEEE 25th International Symposium on Personal, Indoor and Mobile Radio Communications, 2014, pp. 103-107.

[148] P. Banelli, S. Buzzi, G. Colavolpe, A. Modenini, F. Rusek, and A. Ugolini, "Modulation Formats and Waveforms for 5G Networks: Who Will Be the Heir of OFDM?," IEEE Signal Process. Mag., vol. 31, no. 11, pp. 80-93, 2014.

[149] T. A. Thomas, M. Cudak, and T. Kovarik, "Blind Phase Noise Mitigation for a 72 GHz Millimeter Wave System," in IEEE International Conference on Communications (ICC), 2015, pp. 2955-2960.

[150] S. Buzzi, C. D'andrea, T. Foggi, A. Ugolini, and G. Colavolpe, "Single-carrier modulation versus OFDM for millimeter-wave wireless MIMO,” IEEE Trans. Commun., vol. 66, no. 3, pp. 1335-1348, 2018.

[151] R. T. Rakesh, R. Ranjan, S. Gangwar, D. Sen, and G. Das, "Power Efficient OFDM-mmWave Communications with Low Resolution and Subsampling ADC," in IEEE Vehicular Technology Conference, 2017, pp. 1-6.

[152] H. Wang, C. K. Wen, and S. Jin, "Bayesian Optimal Data Detector for mmWave OFDM System with Low-Resolution ADC," IEEE J. Sel. Areas Commun., vol. 35, no. 9, pp. 1962-1979, 2017.

[153] B. Jiang, R. Ye, Y. Huang, S. He, M. Su, and H. Wang, "Comparison of Single Carrier and OFDM on 45GHz Millimeter-wave Communication System with Hardware Impairments," in 19th International Symposium on Wireless Personal Multimedia Communications (WPMC), 2016, pp. 423-428.

[154] K. Roth, L. G. Baltar, M. Faerber, and J. A. Nossek, "Performance analysis of FBMC and CP-OFDM in the presence of phase noise," in EuCNC 2017 - European Conference on Networks and Communications, 2017, pp. 1-5.

[155] Y. Zhang, J. Liu, S. Feng, and P. Zhang, "Pilot Design for Phase Noise Mitigation in Millimeter Wave MIMO-OFDM Systems," in IEEE 85th Vehicular Technology Conference (VTC Spring), 2017, pp. 1-6.

[156] C. Sacchi, T. F. Rahman, N. Bartolomei, S. Morosi, A. Mazzinghi, and F. Ciabini, "Design and Assessment of a CE-OFDM-based mmWave 5G Communication System," in IEEE Globecom Workshops (GC Wkshps), 2016, pp. 1-7.

[157] R. Baldemair et al., "Ultra-dense networks in millimeter-wave frequencies," IEEE Commun. Mag., vol. 53, no. 1, pp. 202-208, 2015.

[158] Ş. Alphan, R. Yang, F. La Sita, and R. L. Olesen, "A Comparison of SC-FDE and UW DFT-s-OFDM for Millimeter Wave Communications," in IEEE International Conference on Communications (ICC), 2018, no. arXiv:1801.09177v1, pp. 1-7.

[159] A. Rahimian, F. Mehran, and R. G. Maunder, "Joint space-time algebraically-interleaved turbo-like Coded Incoherent MIMO systems with optimal and suboptimal MAP probability decoders," in IEEE 4th International Conference on Electronics Information and Emergency Communication, 2013, pp. 1-8.

[160] F. Mehran and R. G. Maunder, "Wireless MIMO systems employing joint turbo-like STBC codes with bit-level algebraically-interleaved URSCs," in 2013 IEEE International Wireless Symposium, IWS 2013, 2013, pp. 2-5. 
[161] D. Kang and W. Oh, "Faster Than Nyquist Transmission with Multiple Turbo-Like Codes," IEEE Commun. Lett., vol. 20, no. 9, pp. 17451747,2016

[162] F. Mehran, K. Nikitopoulos, P. Xiao, and Q. Chen, "Rateless wireless systems: Gains, approaches, and challenges," in IEEE China Summit and International Conference on Signal and Information Processing, 2015, pp. 751-755.

[163] W. Lei and H. Song, "Multi-relay cooperative transmission based on rateless codes and adaptive demodulation," IET Commun., vol. 12, no. 5, pp. 586-594, 2018.

[164] B. Feng, Q. Zhang, and J. Jiao, “An Efficient Rateless Scheme Based on the Extendibility of Systematic Polar Codes,” IEEE Access, vol. 5, no. DOI:10.1109/ACCESS.2017.2762363, pp. 23223-23232, 2017.

[165] D. Gesbert, H. Bolcskei, D. A. Gore, and A. J. Paulraj, “Outdoor MIMO wireless channels: models and performance prediction," IEEE Trans. Commun., vol. 50, no. 12, pp. 1926-1934, 2002.

[166] Matlab, “Correlation Coefficient,” Matlab Documentations, 2017a, 2017. [Online]. Available: https://uk.mathworks.com. [Accessed: 04Sep-2017].

[167] S. Caban and M. Rupp, "Impact of transmit antenna spacing on 2x1 Alamouti radio transmission," IEEE Electron. Lett., vol. 43, no. 4, pp. 198-199, 2008.

[168] J. Luo, J. R. Zeidler, and S. McLaughlin, "Performance analysis of compact antenna arrays with MRC in correlated Nakagami fading channels," IEEE Trans. Veh. Technol., vol. 50, no. 1, pp. 267-277, 2001.

[169] W. C. Lee, “Antenna spacing requirements for a mobile radio base-station diversity,” Bell Syst. Tech. J., vol. 50, no. 6, pp. 1859-1876, 1971.

[170] N. Al-falahy and O. Alani, "Network capacity optimisation in millimetre wave band using fractional frequency reuse," IEEE Access, no. DOI 10.1109/ACCESS.2017.2762338, 2018.

[171] D.-M. R. K. Jain, W. Chiu, and W. R. Hawe, “A Fairness-based Performance Evaluation of Fractional Frequency Reuse in LTE," in 17th International ITG Workshop on Smart Antennas (WSA), 2013, pp. 1-6.

[172] E. H. Cheng-Xiang Wang, Fourat Haider, Xiqi Gao, Xiao-Hu You, Yang Yang, Dongfeng Yuan, Hadi M. Aggoune, Harald Haas, Simon Fletcher, "Cellular Architecture and Key Technologies for 5G Wireless Communication Networks," IEEE Commun. Mag., vol. 52, no. 2, pp. 122-130, 2014.

[173] M. Polese, M. Giordani, M. Mezzavilla, S. Rangan, and M. Zorzi, "Improved Handover Through Dual Connectivity in 5G mmWave Mobile Networks,” IEEE J. Sel. Areas Commun., vol. 35, no. 9, pp. 2069-2084, 2017.

[174] M. Baig, "Calculating a single figure of merit (SFOM) in LTE: a predictor of quality of experience (QoE)," RMIT University, 2011.

[175] I. F. Akyildiz, J. S. M. Ho, and Y.-B. Lin, "Movement-based location update and selective paging for PCS networks," IEEE/ACM Trans. Netw., vol. 4, no. 4, pp. 629-638, 1996.

[176] Y.-B. Lin, W.-R. Lai, and R.-J. Chen, "Performance analysis for dual band PCS networks," IEEE Trans. Comput., vol. 49, no. 2, pp. 148$159,2000$.

[177] N. Al-Falahy and O. Alani, "Supporting Massive M2M Traffic in the Internet of Things Using Millimetre Wave 5G Network," in 9th Computer Science \& Electronic Engineering Conference (CEEC), 2017, pp. 83-88.

[178] S. M. Mitani, T. Kanesan, R. Mohamad, S. Yaakob, and N. E. Farid, "Machine-to-machine communications at millimeter wave frequencies," in IEEE MTT-S International Microwave Workshop Series on: RF and Wireless Technologies for Biomedical and Healthcare Applications, IMWS-Bio, 2014, pp. 1-3.

[179] S. Geng, S. Liu, and X. Zhao, “60-GHz Channel Characteristic Interdependence Investigation for M2M Networks," in 9th International Conference on Communications and Networking in China, 2014, pp. 402-406.

[180] T. Yilmaz and O. B. Akan, "On the use of the millimeter wave and low terahertz bands for Internet of Things," in IEEE World Forum on Internet of Things, WF-IoT 2015 - Proceedings, 2015, pp. 177-180.

[181] S. Scott-hayward and E. Garcia-palacios, "High Definition Video in IEEE 802.15.3c mm-Wave Wireless Personal Area Networks," in 36th Annual IEEE Conference on Local Computer Networks, 2011, pp. 93-100.

[182] M. Xiao et al., "Millimeter Wave Communications for Future Mobile Networks," IEEE J. Sel. Areas Commun., vol. 35, no. 9, pp. 1909$1935,2017$.

[183] ITU, "Minimum requirements related to technical performance for IMT-2020 radio interface(s)," Work. Party 5D, vol. November, no. 5D/TEMP/300(Rev.1), pp. 1-148, 2017.

[184] 5G-PPP, “5G Infrastructure Public Private Partnership,” 2018. [Online]. Available: https://5g-ppp.eu/. [Accessed: 20-Mar-2018].

[185] C. Thuemmler et al., "5G and e-Health," 5G-PPP white paper, no. September, pp. 1-24, 2015.

[186] C. Perfecto, J. Del Ser, and M. Bennis, "Millimeter-Wave V2V Communications: Distributed Association and Beam Alignment," IEEE J. Sel. Areas Commun., vol. 35, no. 9, pp. 2148-2162, 2017.

[187] 5G-PPP Automotive Working Group, “A study on 5G V2X Deployment,” 5G-PPP Initiative, no. Version 1.0, pp. 1-14, 2018.

[188] The 5G Infrastructure Public Private Partnership, "5G Automotive Vision,” 5G-PPP white paper, pp. 1-67, 2015.

[189] IEEE, IEEE 802.11p Standard for information technology -- Amendment 6: wireless access in vehicular environments. 2010.

[190] J. Choi, V. Va, N. González-Prelcic, R. Daniels, C. R. Bhat, and R. W. Heath, "Millimeter-Wave Vehicular Communication to Support Massive Automotive Sensing," IEEE Commun. Mag., vol. 54, no. 12, pp. 160-167, 2016.

[191] B. Antonescu, M. Moayyed, and S. Basagni, "mmWave Channel Propagation Modeling for V2X Communication Systems," in IEEE 28th Symposium on Personal, Indoor, and Mobile Radio Communications (PIMRC), 2017, pp. 1-6.

[192] IEEE, “802.16p-2012 - IEEE Standard for Air Interface for Broadband Wireless Access Systems--Amendment 1: Enhancements to Support Machine-to-Machine Applications," DOI: 10.1109/IEEESTD.2012.6327306, 2012.

[193] C. Pereira and A. Aguiar, “Towards efficient mobile M2M communications: Survey and open challenges,” Sensors, vol. 14, no. 10, pp. 19582-19608, 2014.

[194] M. Palattella et al., "Internet of Things in the 5G Era: Enablers, Architecture and Business Models," IEEE J. Sel. Areas Commun., vol. 34, no. 3, pp. 1-1, 2016.

[195] OneM2M, “oneM2M.” [Online]. Available: http://www.onem2m.org/. [Accessed: 03-Jan-2018].

[196] W. D. de Mattos and P. R. L. Gondim, "M-Health Solutions Using 5G Networks and M2M Communications," IEEE IT Prof., vol. 18, no. 3, pp. 24-29, 2016. 
[197] Agilent Technologies, "Wireless LAN at 60 GHz - IEEE 802 . 11ad Explained," Application Note, pp. 1-28, 2013.

[198] IEEE, “IEEE 802.15 WPAN Task Group 3c (TG3c) Millimeter Wave Alternative PHY,” 2016. [Online]. Available: http://www.ieee802.org/. [Accessed: 09-Dec-2016].

[199] K. Chandra, R. V. Prasad, B. Van Quang, I. G. M. M. Niemegeers, and A. R. Biswas, "Analysis of Fi-Wi Indoor Network Architecture," in Consumer Communications and Networking Conf. (CCNC), 2014, pp. 113-118.

[200] ECMA International, "ECMA-387: High Rate 60GHz PHY, MAC and HDMI PAL Whitepaper," White paper, Ecma/TC48/2010/025, pp. $1-7,2010$.

[201] ECMA International, "Standard ECMA-387 High Rate 60 GHz PHY, MAC and HDMI PALs,” 2010. [Online]. Available: www.ecmainternational.org/. [Accessed: 09-Dec-2016]. 